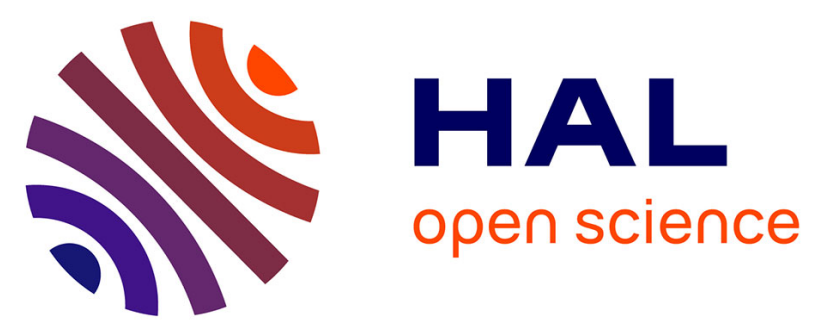

\title{
Les inhumations et les dépôts d'animaux en fosses circulaires du Néolithique récent du sud de la plaine du Rhin supérieur
}

Philippe Lefranc, Anthony Denaire, Fanny Chenal, Rose-Marie Arbogast

\section{To cite this version:}

Philippe Lefranc, Anthony Denaire, Fanny Chenal, Rose-Marie Arbogast. Les inhumations et les dépôts d'animaux en fosses circulaires du Néolithique récent du sud de la plaine du Rhin supérieur. Gallia Préhistoire - Préhistoire de la France dans son contexte européen, 2010, 52, pp.61-116. 10.3406/galip.2010.2471 . halshs-00590543

\section{HAL Id: halshs-00590543 \\ https://shs.hal.science/halshs-00590543}

Submitted on 2 Jan 2020

HAL is a multi-disciplinary open access archive for the deposit and dissemination of scientific research documents, whether they are published or not. The documents may come from teaching and research institutions in France or abroad, or from public or private research centers.
L'archive ouverte pluridisciplinaire HAL, est destinée au dépôt et à la diffusion de documents scientifiques de niveau recherche, publiés ou non, émanant des établissements d'enseignement et de recherche français ou étrangers, des laboratoires publics ou privés.

\section{(ㅇ)(1) $\$$}

Distributed under a Creative Commons Attribution - NonCommercial - NoDerivatives 44.0 


\title{
LES INHUMATIONS ET LES DÉPÔTS D'ANIMAUX EN FOSSES CIRCULAIRES DU NÉOLITHIQUE RÉCENT DU SUD DE LA PLAINE DU RHIN SUPÉRIEUR
}

\author{
Philippe LEFRANC*, Anthony DenAIRE**, Fanny CHENAL ${ }^{* * *}$ et Rose-Marie ARBOGAST ${ }^{* * * * *}$
}

\begin{abstract}
Mots-clés. Inhumation en fosses de plan circulaire, sépulture plurielle, manipulation, Néolithique moyen, Michelsberg, Munzingen, Alsace, Pays de Bade.

Résumé. Cette contribution propose une analyse synthétique des dépôts humains et animaux en fosses de plan circulaire du Néolithique récent du sud de la plaine du Rhin supérieur. Elle s'appuie sur de nouvelles données, souvent inédites, issues des opérations de fouilles préventives. L'important corpus régional permet de mettre en évidence plusieurs traits récurrents autorisant l'approche d'un système complexe où coexistent des inhumations régulières simples, des dépôts simultanés associant individus en position régulière et corps en position désordonnée, des dépôts successifs et des dépôts secondaires. La découverte de corps humains et de carcasses d'animaux, ayant fait l'objet de manipulations postdécompositionnelles, permet de restituer une gestuelle complexe pouvant s'apparenter à celle que l'on observe dans de nombreux fossés d'enceintes. Nous nous interrogeons également, en nous appuyant sur l'association d'un corps non apprêté et d'un dépôt d'objets en cuivre, sur l'éventuelle existence de dépôts humains réalisés hors du cadre funéraire. Une confrontation du modèle avec les données issues d'autres régions, notamment la vallée du Rhône et l'Allemagne, permet d'insister sur la cohérence du phénomène des dépôts en fosses circulaires d'une extrémité à l'autre de son aire de répartition.
\end{abstract}

Key-words. Inhumation in a circular pit, multiple burial, manipulation, Middle Neolithic, Michelsberg, Munzingen, Alsace, Pays de Bade.

\begin{abstract}
This article presents a synthetic analysis of the human and animal burials in circular pits attributed to the late Neolithic of the southern plain of the Upper Rhine. It is based on new, often unpublished, data resulting from preventive excavations. The large regional corpus allows us to reveal several recurrent traits that permit the study of a complex system with the coexistence of regular, simple inhumations, simultaneous burials associating individuals in a regular position and bodies in disorderly positions, successive burials and secondary burials. The discovery of human bodies and animal carcasses, which were the object of post-decompositional manipulations, allows us to reconstruct a complex act that could be similar to that observed in numerous enclosure wall trenches. We also address the question of the possible existence of human burials realized outside of the funerary context, based on the association of a non prepared body and a deposit of copper objects. A confrontation of the model with data from other regions, notably the Rhone Valley and Germany, allow us to insist on the coherence of the phenomenon of burials in circular pits from one extremity to the other of its zone of distribution.
\end{abstract}

Translation: Magen O'FARRELL

\footnotetext{
* UMR 7044 du CNRS, Inrap, Centre archéologique de Strasbourg, 10 rue d'Altkirch, F-67100 Strasbourg. Courriel : philippe.lefranc@inrap.fr

** UMR 7044 du CNRS, Antea-Archéologie, 11 rue de Zurich, F-68440 Habsheim. Courriel : anthony.denaire@antea-archeologie.com

*** Inrap, Centre archéologique de Strasbourg, 10 rue d'Altkirch, F-67100 Strasbourg. Courriel : fanny_chenal@hotmail.com

***** UMR 7044 du CNRS, Misha, 5 allée du général Rouvillois, F-67083 Strasbourg. Courriel : rosemarie.arbogast@unibas.ch
} 
L'accroissement important, ces dernières années, du corpus des inhumations et des dépôts d'animaux en fosses de plan circulaire fait aujourd'hui du sud de la plaine du Rhin supérieur, et en particulier, de l'Alsace, une des régions les mieux documentées d'Europe occidentale pour ce phénomène. L'accumulation de nouvelles données, toutes issues d'opérations de fouilles préventives, nous semble justifier une nouvelle tentative de synthèse régionale s'inscrivant à la suite des récents travaux consacrés à cette question (Denaire, 2007; Boës, 2007; Lefranc et al., 2007; Jeunesse, 2010). Les données inédites présentées ici ont, pour la plus grande partie, été recueillies sur des sites dont l'un ou l'autre des auteurs a conduit la fouille et/ou l'étude. Cette contribution aborde les questions de la chronologie, de la nature des creusements, de la composition des dépôts et du mobilier funéraire, et se propose essentiellement de faire un point sur les problématiques archéologiques: les questions complexes de taphonomie ne seront que peu discutées dans le détail; nous nous référerons sur ces points précis aux travaux des anthropologues de terrain ouvrant en Alsace et disponibles dans diverses publications et rapports de fouille.

\section{CADRE CHRONOLOGIQUE ET GÉOGRAPHIQUE}

Dans le sud de la plaine du Rhin supérieur, les débuts de la pratique de l'inhumation en fosses circulaires peuvent être fixés dès la fin du Néolithique moyen, plus précisément au cours de l'horizon épiroessénien; elle se généralise au Néolithique récent, avant, semble-t-il, de disparaître à la période suivante, période, il est vrai, très peu documentée. La fourchette chronologique prise en compte court donc sur un millénaire, entre 4400 et 3400 av. J.-C. environ.

Le choix du cadre géographique, entre Vosges et Forêt Noire, se justifie par la présence, de part et d'autre du Rhin, de groupes et de cultures identiques, le fleuve ne constituant à aucun moment une frontière (fig. 1). Côté alsacien, la totalité de la plaine a été prise en compte, du nord du Jura à la confluence Rhin-Lauter. Côté badois, la zone étudiée est le pendant de celle retenue sur la rive gauche, ce qui en exclut les sites de la région de Karlsruhe, notamment Bruchsal et Untergrombach. Bien qu'elle soit occupée, au moins en partie, par les mêmes groupes que ceux présents dans le sud de la plaine rhénane, le nord de la Franche-Comté a en revanche été délaissé faute de données.

\section{ORIGINE ET EXTENSION DU PHÉNOMÈNE DU DÉPÔT EN FOSSES DE PLAN CIRCULAIRE}

La question des inhumations en fosses circulaires du Néolithique récent, amplement discutée, a fait l'objet de plusieurs travaux de synthèse aux conclusions divergentes. Il n'est pas dans notre propos de reprendre l'historique des recherches ni d'établir un catalogue des hypothèses. Nous rappellerons que les conclusions émises par les divers auteurs s'étant penchés sur la question peuvent être réduites à deux propositions opposées: la première, inférant de la grande diversité des pratiques caractérisant le domaine d'étude, réfute le caractère funéraire des inhumations en fosses circulaires (Nickel, 1998) et les considère comme le reflet d'une pratique marginale se dérobant à la synthèse; la seconde voit dans ces inhumations de véritables sépultures installées dans des creusements spécialement aménagés pour les funérailles (Lichardus, 1986; Schweitzer, 1987). Les travaux les plus récents, plus nuancés dans leurs conclusions et bénéficiant de données de terrain renouvelées, mettent l'accent sur l'existence d'un système cohérent d'où se dégage un certain nombre de faits récurrents; très récemment, C. Jeunesse a proposé une synthèse dans laquelle il s'attache à dégager les constantes et les dénominateurs communs caractérisant ce mode d'inhumation (Jeunesse, 2010). Il propose d'assimiler à de véritables sépultures les dépôts en fosses circulaires et replace cette pratique particulière dans un large contexte européen où ces sépultures dessinent une «province funéraire» s'étendant de la vallée du Rhône à l'est de l'Europe centrale. Dans l'état actuel de la recherche, il semble que cette pratique apparaisse en contexte chasséen avant de se diffuser au sein du Michelsberg puis, par l'intermédiaire de cette culture, dans le Münchshöfen de Bavière, le Lengyel tardif et la culture des "gobelets à col en entonnoir» (Trichtbecher Kultur) (Jeunesse, 2010).

L'hypothèse d'une origine chasséenne du phénomène repose en partie sur les datations hautes proposées pour les inhumations en fosses rondes des sites des Plots à Berriac, dans l'Aude (Duday, Vaquer, 2003) et du Crès à Béziers dans l'Hérault (Loison et al., 2003), attribuées au Chasséen ancien. La découverte récente de deux inhumations en fosses circulaires dans le Valais, à la Colline du Grand Pré à Saint-Léonard (Mariéthoz, 2007), pourrait appuyer cette proposition. Ces deux inhumations - une inhumation secondaire d'enfant et une inhumation primaire de femme 


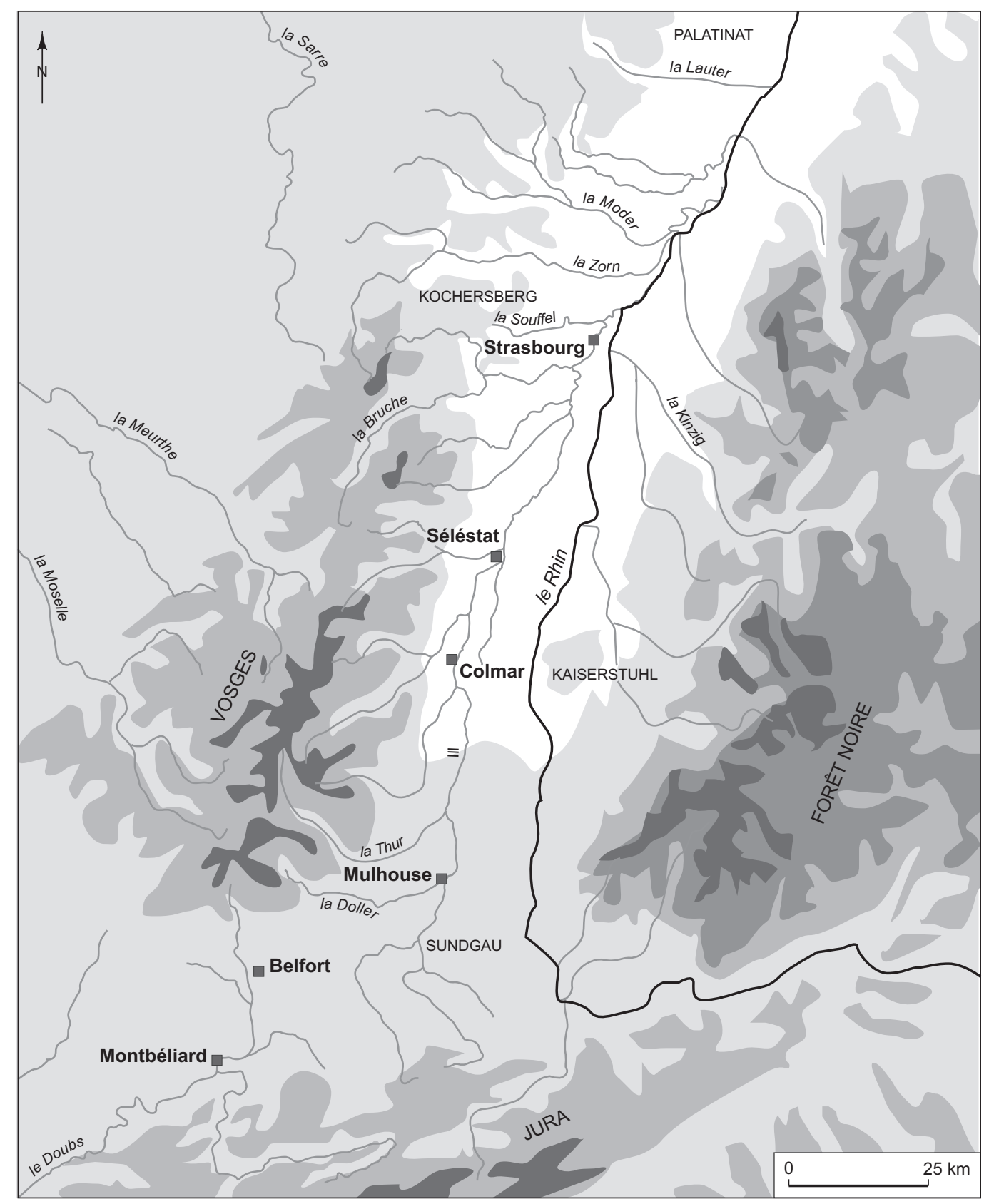

Fig. 1 - Le sud de la plaine du Rhin (DAO: Ph. Lefranc, Inrap).

âgée -, datées entre 4320 et 4050 av. J.-C. sont imputées à une influence chasséenne, également reconnue dans le mobilier recueilli dans les niveaux les plus anciens de l'habitat. Dans les deux cas, les corps ont été déposés dans des creusements circulaires présentant également des niveaux de comblement constitués de rejets domestiques.

D'après les données aujourd'hui disponibles, les premières manifestations de cette pratique en Alsace et dans le sud du pays de Bade sont attribuées peut-être au Bischheim (Stöckl, Neubauer-Saurer, 1990; Jeunesse et al., 2003) et sûrement au BORS ${ }^{1}$ (Lefranc, 2001) que l'on peut dater entre 4600 et 4350 pour le premier et entre 4300 et 4050 4000 av. J.-C. pour le second, soit une fourchette au moins

1. Bischheim occidental du Rhin supérieur, ancien groupe d'Entzheim (Jeunesse et al., 2003). 
aussi ancienne, sinon plus, que celle admise pour les inhumations en fosses circulaires chasséennes. Il n'est pas possible de préciser si cette pratique apparaît simultanément dans les régions occupées par le Chasséen et la nôtre ou si nous avons affaire à une diffusion très rapide des premières vers la seconde. Compte tenu de l'imprécision des datations actuelles, les deux scénarios restent possibles.

\section{LE CORPUS}

\section{PRÉSENTATION GÉNÉRALE}

D'après la documentation disponible, 77 fosses circulaires du Néolithique moyen et récent avec des os humains ont été mises au jour dans le sud de la plaine du Rhin supérieur. Elles proviennent de 28 sites. Quinze sont localisés en Basse-Alsace, dix en Haute-Alsace et trois dans le Bade. Comme la période qui nous intéresse ne compte pas seulement des inhumations en fosses circulaires mais que certains défunts ont également été enterrés dans des fossés, des minières, des fentes, des fosses ovales et irrégulières ainsi que dans de «vraies» fosses sépulcrales ajustées au corps du défunt, il est apparu judicieux de prendre en compte l'ensemble des structures accueillant des inhumations. Le corpus est ainsi porté à 51 sites sur lesquels 127 structures ont livré les restes de plus de 193 individus. À une exception près, la pratique de l'inhumation est exclusive. Dans la majorité des cas, il s'agit de corps inhumés entiers ou presque (159 cas), loin devant les os isolés ( 27 cas) et les segments anatomiques (7 cas).

La répartition géographique de ces découvertes n'est pas homogène (fig. 2). La Basse-Alsace, et en particulier les placages loessiques localisés à l'ouest de Strasbourg, est la région la mieux dotée, avec 28 sites, contre 13 pour toute la Haute-Alsace et 10 pour le sud du Bade. En nombre de structures, la Basse- et la Haute-Alsace font en revanche presque jeu égal avec respectivement 60 et 53 fosses, mais toujours loin devant le sud du Bade (14 structures).

La qualité de la documentation est très variable d'un site à l'autre. Elle est souvent médiocre lorsqu'il s'agit de découvertes anciennes qui bénéficient rarement de descriptions précises alors que les trouvailles récentes font l'objet d'analyses, notamment anthropologiques, poussées (Blaizot, 2001; Lefranc, Boës, 2006; Boës, 2007; Lefranc, Chenal, 2010). Par ailleurs, la datation de ces inhumations pose souvent problème. L'attribution au Néolithique récent est souvent hypothétique. Dans de trop nombreux cas, elle ne s'appuie en effet que sur le contexte de découverte et

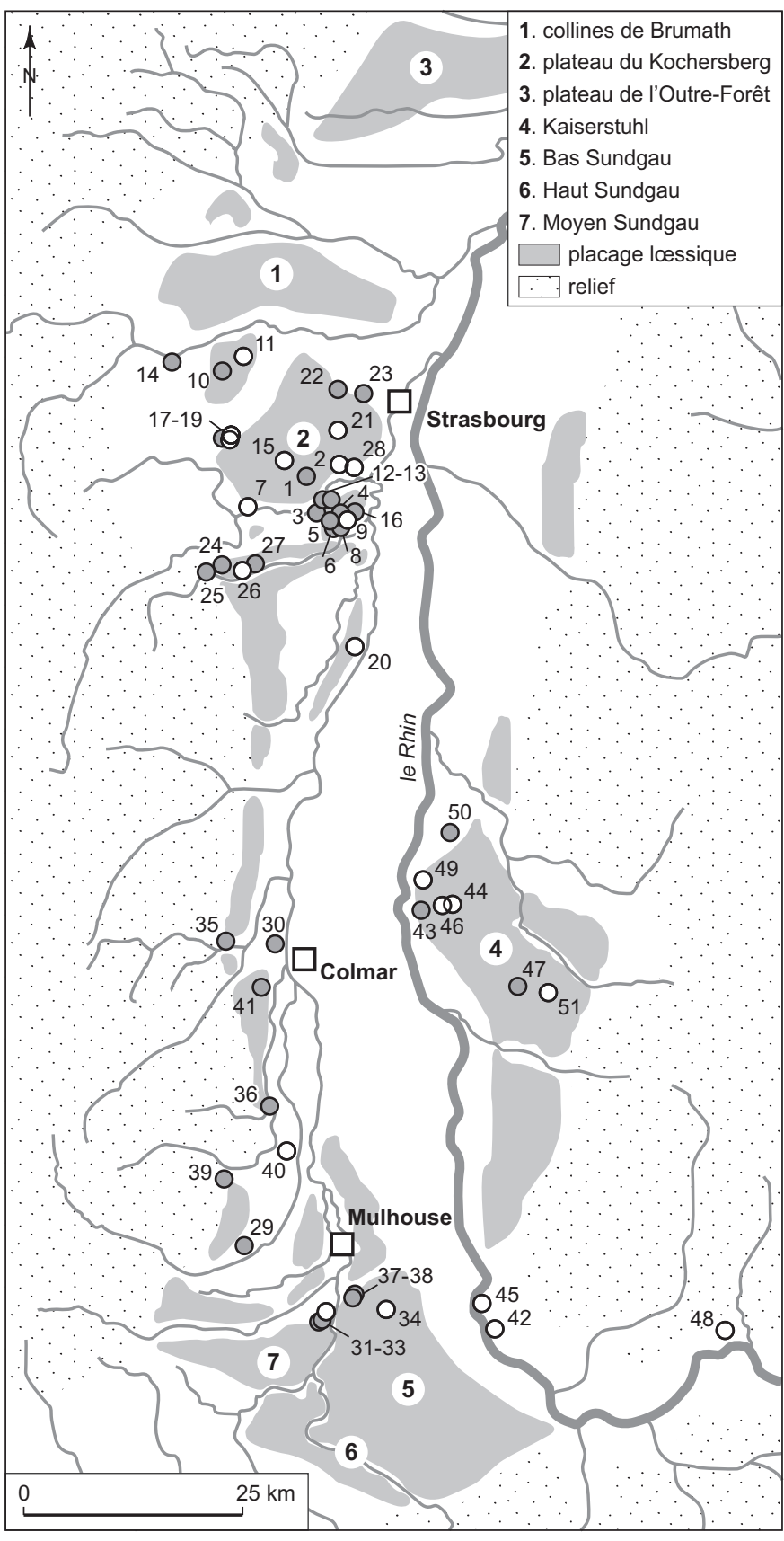

Fig. 2 - Carte de localisation des sites mentionnés dans le texte pour le sud de la plaine du Rhin supérieur (les numéros renvoient aux ensembles recensés dans le catalogue des sites, p. 95)

(DAO: A. Denaire, Antea-Archéologie).

l'absence, sur les sites concernés, de certaines étapes de la Protohistoire pendant lesquelles l'inhumation en fosse circulaire est aussi pratiquée. Bien entendu, une telle argumentation reste sujette à caution, même si elle n'est pas totalement infondée. 


\section{DATATION ET ATTRIBUTION GHRONOLOGIQUE}

En Alsace et dans le sud du pays de Bade, plus d'une centaine de sites sont recensés pour la fin du Néolithique moyen et un peu moins de 80 pour le Néolithique récent. Nombre d'entre eux ne sont connus que par des découvertes ponctuelles, mais quelques-uns, étudiés sur des surfaces importantes, ont livré une documentation de qualité permettant de reconnaître assez finement l'évolution stylistique des nombreux groupes et cultures qui s'y succèdent. La fin du Néolithique moyen et la transition avec le Néolithique récent ayant récemment fait l'objet d'une synthèse, nous y renvoyons le lecteur pour plus de détails (Jeunesse et al., 2003). En ce qui concerne le Néolithique récent, les travaux de synthèse sont plus anciens, mais leurs conclusions restent valables (Jeunesse, 1989). Nous nous contenterons donc ici de brosser à grands traits l'évolution chronologique de notre aire d'étude, marquée par plusieurs ruptures, particulièrement en Basse-Alsace.

La seconde partie du Néolithique moyen voit se succéder en Alsace et dans le sud du Bade le groupe de Bischheim puis de Bruebach-Oberbergen qui lui est génétiquement lié (fig. 3). Très rapidement, le BORS s'implante en Basse-Alsace, interrompant brutalement l'évolution du Bruebach-Oberbergen, ce dernier ne subsistant plus alors qu'en Haute-Alsace. Lapparition du BORS dans la vallée du Rhin doit être imputée à des influx occidentaux se poursuivant avec l'installation du Michelsberg ancien du Rhin supérieur en Basse-Alsace. Probablement sous l'impulsion de groupes extérieurs, le Michelsberg moyen (MK III) se met ensuite en place. La phase récente du Michelsberg (IV) est interrompue par l'émergence du Munzingen (étapes $\mathrm{B}$ et $\mathrm{C}$ ), groupe originaire de la région du Kaiserstuhl et du nord de la Haute-Alsace (Lefranc, Jeunesse, 2001).

En Haute-Alsace, l'irruption du BORS est plus tardive. Contrairement à la Basse-Alsace, ce style n'y est pas remplacé par le Michelsberg ancien mais évolue pour donner naissance au Munzingen. Si l'étape A de cette culture est bien documentée, son évolution demeure assez mal connue dans le sud de l'Alsace. Quelques éléments Munzingen B y sont bien signalés, mais ils ne sont pas sans trouver d'affinités dans des séries plus anciennes. Il est possible que l'évolution du Munzingen n'y soit pas tout à fait identique à celle de la région du Kaiserstuhl et du nord de la Haute-Alsace, mais les données précises font défaut et les datations radiocarbone entretiennent l'ambiguité sur cette question.

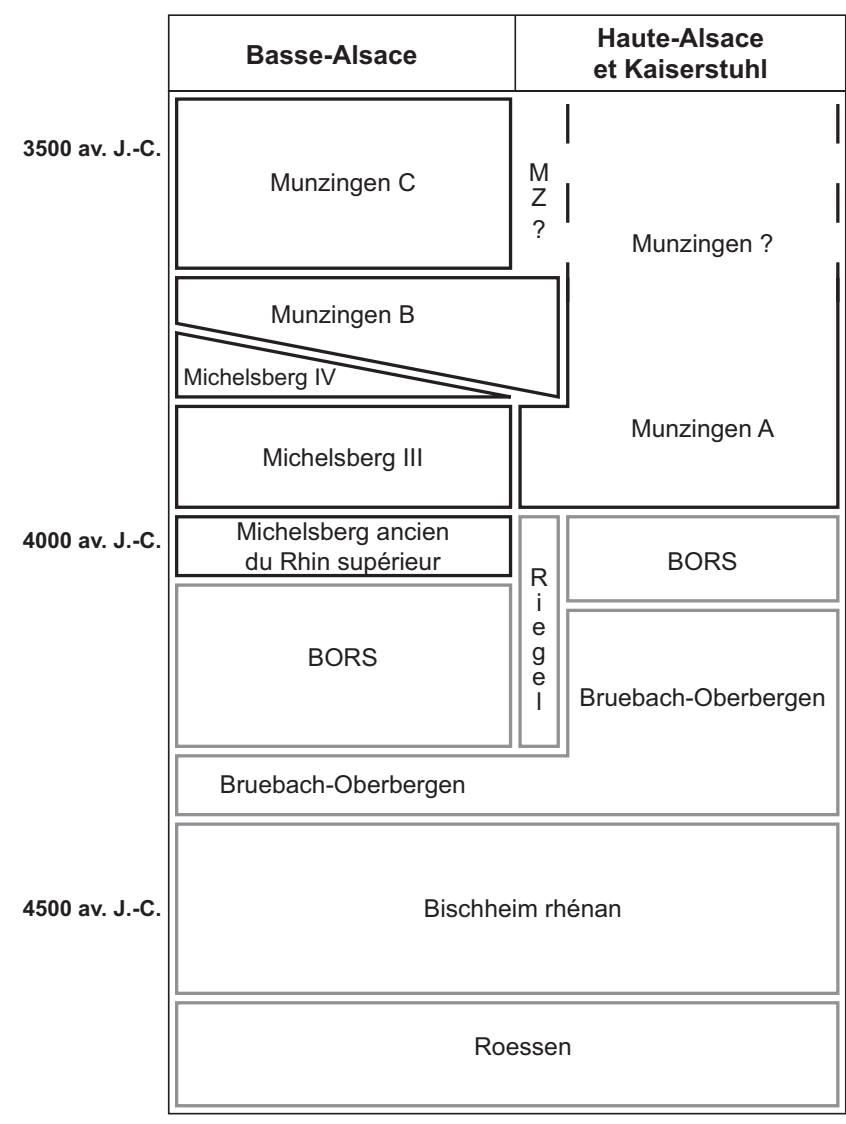

Fig. 3 - Tableau chronologique de la période 4800-3400 av. J.-C. dans le sud de la plaine du Rhin supérieur (DAO: A. Denaire, Antea-Archéologie).

L'évolution et la succession des groupes et cultures archéologiques sont donc bien connues en Alsace. En revanche, leur datation absolue pose problème. Pourtant une cinquantaine de dates radiocarbone ont été réalisées, mais le plus souvent sur des échantillons provenant justement de fosses à inhumation dépourvues de mobilier caractéristique. De plus, parmi les quelques exceptions, rares sont celles utilisables une fois éliminées les dates dont le contexte de découverte pose problème, celles dont l'intervalle est trop large ( $>100$ ans) et enfin, celles qui sont visiblement trop jeunes ou trop anciennes (Jeunesse, Sainty, 1987). Ainsi, aucune date ne peut être retenue pour le Bischheim, le Bruebach-Oberbergen, le BORS, le Michelsberg III et IV et le Munzingen A et B. Les seules datations acceptables sont celles du Lavoir à Matzenheim pour le Michelsberg ancien du Rhin supérieur (4040-3960 av. J.-C. ${ }^{2}$ ) et de Forlen à Geispolsheim,

2. Date calibrée à $1 \sigma$. 


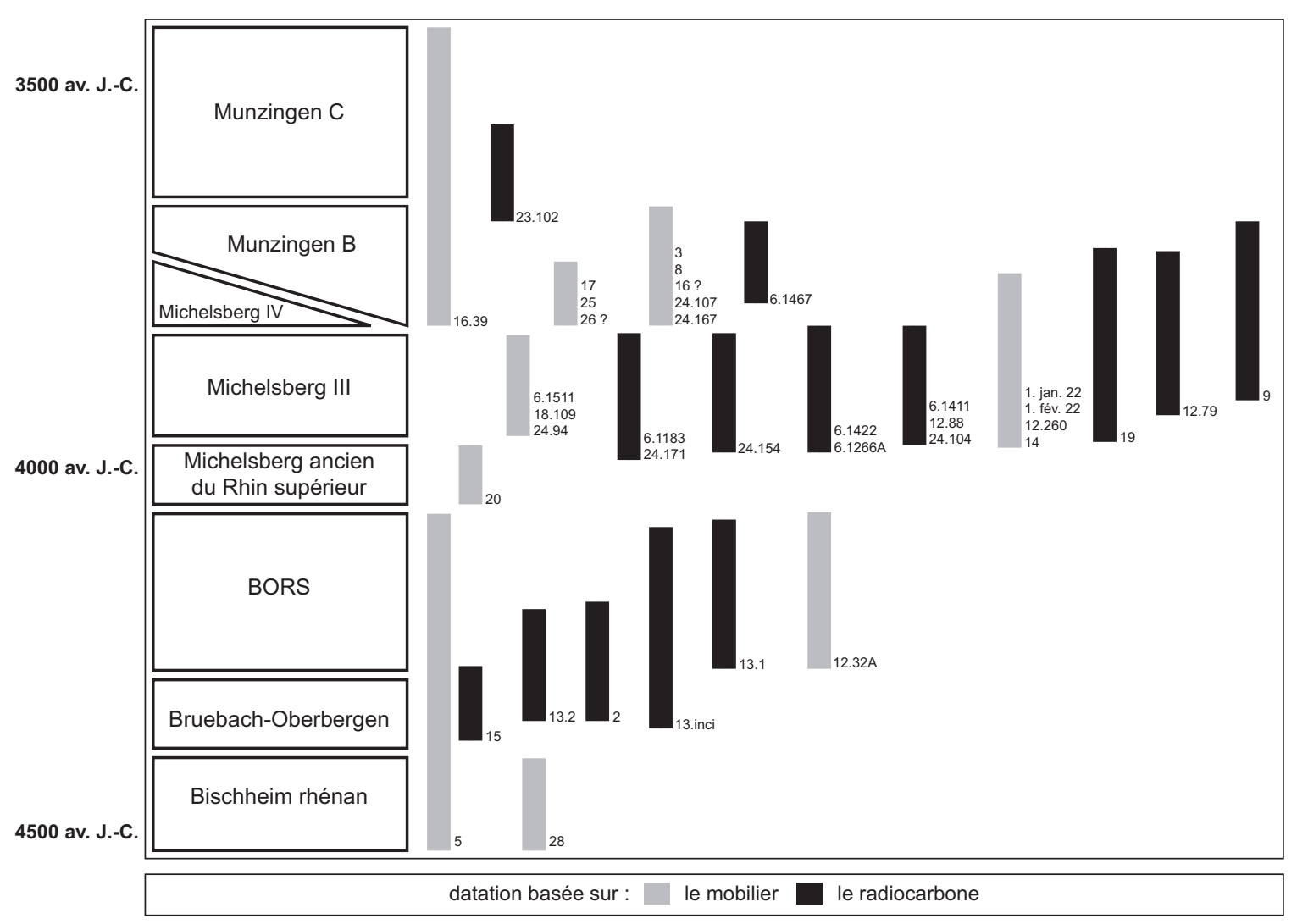

Fig. 4 - Tableau récapitulatif des datations retenues pour les inhumations de la fin du Néolithique moyen et du Néolithique récent en Basse-Alsace (les numéros renvoient aux ensembles recensés dans le catalogue des sites, p. 95; le numéro après le point renvoie à l'inhumation correspondante au sein de l'ensemble) (DAO: A. Denaire, Antea-Archéologie).

pour le Munzingen C (3640-3380 av. J.-C.). Celles de la nécropole d'Ungersheim (catalogue, site $n^{\circ} 40$ ) peuvent toutefois servir de point de repère pour fixer l'extrême fin du Néolithique moyen dans ce secteur (4040-3965 av. J.-C.). Il est donc indispensable, pour dater les articulations de cette évolution chronologique, de s'appuyer sur les données des régions voisines mieux dotées, avec tous les biais qu'un tel exercice comporte. Ainsi, pour le Bischheim, le cumul des différentes datations disponibles permet de retenir la fourchette de 4600-4350 av. J.-C. (Denaire, 2009). Les études dendrochronologiques d'Egolzwil 3 donnent la date de 4282-4275 av. J.-C. pour l'étape ancienne du Bruebach-Oberbergen (Capitani, 2007). Quant aux débuts du BORS en Basse-Alsace, ils peuvent être situés vers 4300 av. J.-C. par comparaison avec la chronologie du groupe de Schwieberdingen (Jeunesse et al., 2003). Rien ne permet en revanche de dater l'arrivée du BORS en HauteAlsace, même s'il est difficilement concevable qu'elle se fasse avant 4200-4100 av. J.-C. Comme le Munzingen A peut être rapproché du Michelsberg III, il faut nécessairement le placer après 4000 av. J.-C., ce que confirme par ailleurs la datation de la nécropole d'Ungersheim. L'étape récente du Michelsberg (IV) commencerait vers 3800 av. J.-C. et le Munzingen B peu après cette date si on se fie aux datations du site de Klingenberg à Heilbronn (Seidel, 2005) .

Cette esquisse de chronologie absolue de la fin du Néolithique moyen et du Néolithique récent du sud de la plaine du Rhin supérieur est synthétisée sur la figure 3. Sans être définitive, elle permet toutefois de préciser l'attribution culturelle des ensembles datés par ${ }^{14} \mathrm{C}$ (fig. 4 et fig. 5), et ainsi de redonner une certaine profondeur historique à la pratique de l'inhumation en fosses circulaires dans cette région. Cet exercice a toutefois ses limites. Outre les incertitudes liées à l'utilisation de données provenant des régions voisines pour établir un cadre de comparaison - un décalage est toujours possible entre régions -, il ne faut pas oublier qu'il est hasardeux de se baser sur une date radiocarbone pour déterminer à quelle culture ou quelle étape appartient l'ensemble concerné. Le décalage entre les dates radiocarbone des tombes de la Rocade ouest 


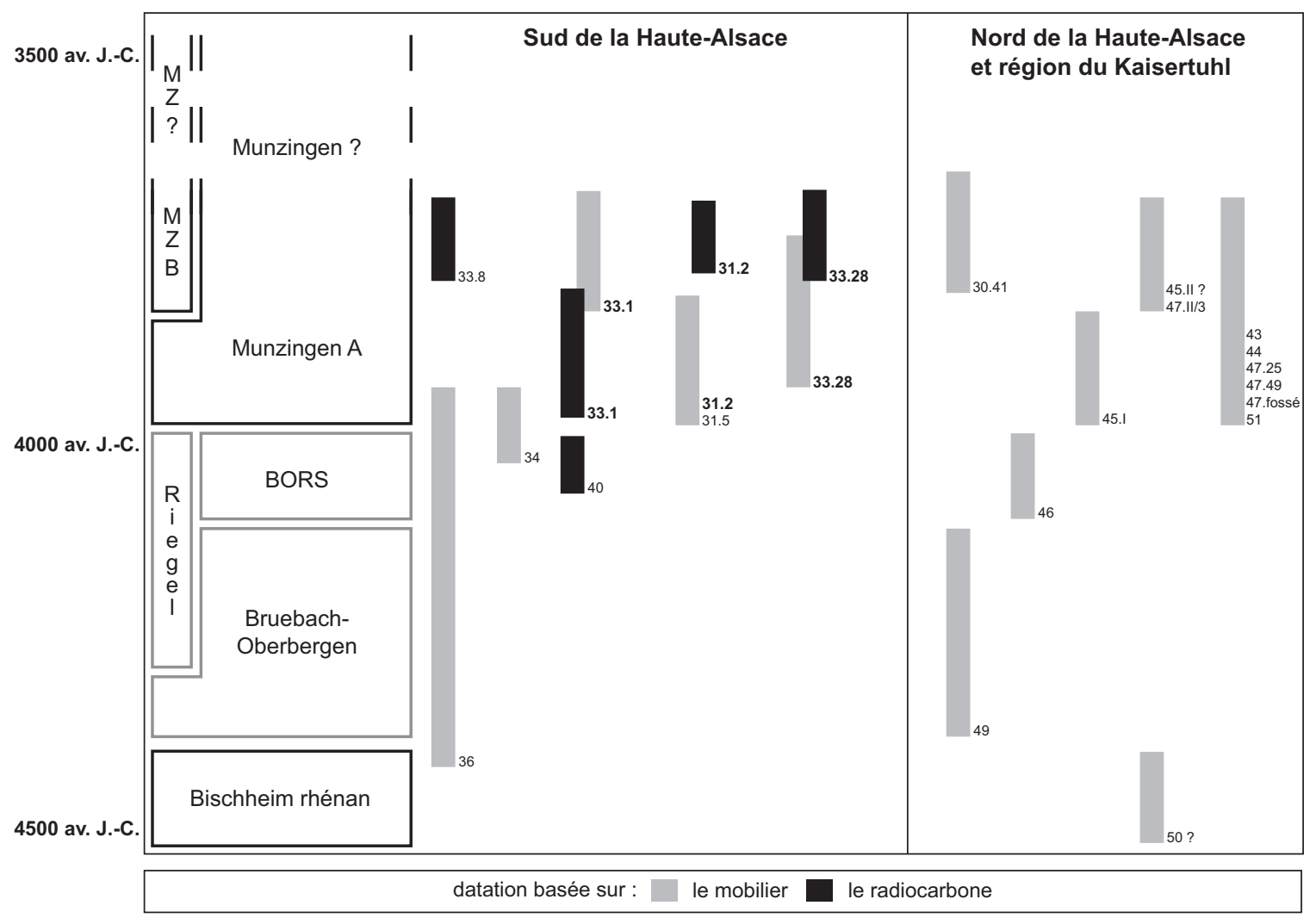

Fig. 5 - Tableau récapitulatif des datations retenues pour les inhumations de la fin du Néolithique moyen et du Néolithique récent en Haute-Alsace et dans le sud du Bade (les numéros renvoient aux ensembles recensés dans le catalogue des sites, p. 95; le numéro après le point renvoie à l'inhumation correspondante au sein de l'ensemble) (DAO: A. Denaire, Antea-Archéologie).

à Didenheim/Morschwiller-le-Bas et celles admises pour leur mobilier est là pour le rappeler (fig. 5). D'autres biais existent, notamment le rajeunissement qui semble affecter certaines mesures ${ }^{14} \mathrm{C}$ sur os (Denaire, 2009).

\section{LA FIN DU V V MILLÉNAIRE ET LA TRANSITION NÉOLITHIQUE MOYEN-RÉCENT (4400-4000 AV. J.-C.)}

De façon générale, la transition entre le Néolithique moyen et le Néolithique récent reste assez mal documentée en ce qui concerne les pratiques funéraires. Durant les 600 ans qui séparent les débuts du Bischheim et ceux du Michelsberg moyen, seules 34 inhumations sont signalées en Alsace et dans le Bade, corpus à comparer aux 400 tombes d'horizon Grossgartach et Roessen fouillées dans la région.

En Basse-Alsace, les quatre tombes de Koenigshoffen à Strasbourg montrent que les traditions funéraires antérieures se maintiennent (site $\mathrm{n}^{\circ} 28$ ): les individus sont inhumés sur le dos en position allongée, orientés nordouest-sud-est avec la tête au nord-ouest. Le mobilier funéraire se compose d'éléments de parure, de mobilier lithique et de céramique.

La seule tombe du groupe de Bruebach-Oberbergen connue en Basse-Alsace est celle du Complexe sportif à Ittenheim récemment fouillée (site $\left.\mathrm{n}^{\circ} 15\right)$. Cette attribution repose sur une date radiocarbone. Il s'agit de la tombe d'un enfant en bas âge inhumé allongé sur le dos et accompagné d'un mobilier en bois de cerf. Cette tombe ne diffère des précédentes que par l'orientation sud-est-nord-ouest du défunt.

Les rares données disponibles pour le groupe de BORS sont divergentes. La sépulture récemment mise au jour aux Terres de la chapelle à Entzheim (site $n^{\circ} 5$ ), attribuée à l'horizon Bischheim/BORS sans précision, présente les mêmes caractères que celle d'Ittenheim. Si on retient pour la datation de la tombe d'Entzheim l'horizon BORS, elle témoignerait alors du maintien des pratiques funéraires du Néolithique moyen régional. En revanche, toutes les autres inhumations attribuées à ce groupe indiquent une rupture profonde. C'est en effet au BORS qu'appartient 
la première inhumation en fosses circulaires de BasseAlsace. Il s'agit du silo 32A du site d'Altmatt à Holtzheim ( site $\mathrm{n}^{\circ} 12$ ). Les restes d'un individu placé en position fléchie sur le côté gauche y ont été découverts à environ $40 \mathrm{~cm}$ au-dessus du fond. Il est orienté selon un axe nordsud avec la tête au nord. Le mobilier recueilli se compose d'un gobelet décoré déposé au même niveau que le corps. Une seconde inhumation en silo fouillée sur le site de la Sablière Oesch à Entzheim peut éventuellement être datée de cet horizon chronologique. Toutefois, son attribution au BORS reste hypothétique, faute de mobilier (site $n^{\circ} 4$ ). D’après les résultats du radiocarbone, la tombe récemment mise au jour au cours d'une opération de diagnostic archéologique sur la commune d'Eckbolsheim (site $n^{\circ} 2$ ) appartient vraisemblablement au BORS. La fosse sépulcrale de plan subquadrangulaire aux angles arrondis, orientée est-ouest, contenait le corps d'une femme adulte reposant en position fléchie sur le côté droit, tête à l'est. Sans équivalent en Alsace, cette sépulture «régulière» trouve de bons parallèles dans le Bassin parisien, en contexte Michelsberg ancien, qu'il s'agisse de la position fléchie sur le côté droit ou de l'orientation est-ouest: citons notamment, et sans souci d'exhaustivité, les sépultures Michelsberg ancien récemment étudiées à Beaurieux dans l'Aisne (Colas et al., 2007; Thévenet, 2007) et à la Noue Fenard à Vignely en Seine-et-Marne (Lanchon et al., 2006). Il n'est peut-être pas illégitime d'identifier à Eckbolsheim, l'impact en milieu funéraire des influences occidentales dont témoignent clairement les assemblages céramiques régionaux (Jeunesse et al., 2003).

D'après les datations radiocarbone réalisées, les restes humains du fossé interrompu des Sablières Réunies à Holtzheim appartiendraient également au BORS, même s'il n'est pas possible d'exclure définitivement une datation plus haute, c'est-à-dire Bruebach-Oberbergen (site no 13). Le fossé interne a livré des os humains erratiques - principalement des éléments de crâne -, des ossements brûlés appartenant à au moins deux individus, et deux squelettes fortement remaniés et démembrés (dépôts 1 et 2), que l'on doit peut-être interpréter comme des dépôts secondaires.

Pour le Michelsberg ancien du Rhin supérieur, les données sont encore plus ténues. La seule inhumation sûrement attribuée à ce faciès est celle du Lavoir à Matzenheim (site $n^{\circ} 20$ ). Le défunt a été enterré allongé sur le dos dans une fosse spécialement creusée à cet effet. Le mobilier funéraire se compose de deux vases déposés à ses pieds et de deux artefacts en silex retrouvés au niveau de l'avant-bras gauche. Si la position du corps, son orientation nord-ouest- sud-est et la disposition du mobilier rappellent les pratiques Roessen ou Bischheim, en revanche l'architecture de la fosse sépulcrale ne trouve pas de parallèles en Alsace.

En Haute-Alsace, la fin du Néolithique moyen n'a guère livré de vestiges funéraires. La découverte la plus ancienne est celle réalisée dans un des silos du site de Trummelmatten à Merxheim (site $n^{\circ} 36$ ) où figuraient un crâne pourvu de sa mandibule ainsi que les éléments d'un collier permettant de proposer une datation large allant de la fin du Bischheim au début du Munzingen.

La sépulture d'Eschentzwiller dans le Sundgau, que l'on doit sans doute attribuer à la transition des Ve et IV millénaires, reste un cas isolé difficile à commenter (site $\left.n^{\circ} 34\right)$. L'individu est couché en position repliée sur le côté droit, tête au sud. La morphologie du récipient déposé auprès du défunt est très proche des types Pfyn ancien de Hornstaad «Hörnle 1» (Dieckmann, 1985; Dieckmann et al., 2007). Cette empreinte orientale, que l'on décèle également à Magstatt-le-Bas (Wolf, 1979), évoque d'autres découvertes, notamment les bouteilles de type Lutzengüetle de la Grotte de la Tuilerie à Gondenans-Montby (Pétrequin, 1972) qui témoignent, probablement au même titre que les deux ensembles sundgauviens, de la pénétration d'influences Pfyn ancien en direction des sites du Néolithique moyen bourguignon de la Trouée de Belfort ${ }^{3}$.

La fouille récente d'une petite nécropole à Ungersheim (site $\mathrm{n}^{\circ} 40$ ) a permis de montrer que les traditions funéraires du Mittelneolithikum rhénan se sont maintenues dans le sud de la Haute-Alsace jusqu'aux années 4040-3965 av. J.-C. (date radiocarbone calibrée à $1 \sigma$; Lefranc et al., 2009). Les 17 individus ont été inhumés sur le dos, membres inférieurs en extension, sur un axe sud-est-nord-ouest, tête au sudest. Les seules différences notables avec les nécropoles du Néolithique moyen résident dans la relative pauvreté des dépôts funéraires et dans l'orientation des corps tête au sudest. Sur ce dernier point, il faut tout de même nuancer notre propos en rappelant que cette orientation sud-est-nordouest existe dès la première partie du Néolithique moyen et qu'il n'est pas impossible qu'elle constitue la norme en la matière pour cette région (Denaire, Jeunesse, 2010).

La région du Kaiserstuhl est également pauvre en vestiges funéraires de la fin du Néolithique moyen. La tombe la plus ancienne serait celle de Ober Endinger Weg à Weisweil, attribuée au groupe de Bischheim d'après le décor d'un

3. Rappelons que les cultures de Pfyn et du Néolithique moyen bourguignon entretiennent, dès le tournant des $\mathrm{V}^{\mathrm{e}}$ et $\mathrm{IV}^{\mathrm{e}}$ millénaires, des relations étroites basées sur l'exportation des lames de hache en pélitequartz (Pétrequin, Jeunesse, 1995). 
grand tesson provenant du remplissage ( datation se confirmait, il s'agirait alors de la plus ancienne inhumation en fosse circulaire du sud de la plaine du Rhin supérieur. Le défunt reposait sur le dos, en position désordonnée, et orienté sud-sud-est-nord-nord-ouest. Si cette inhumation marque une rupture nette dans les pratiques funéraires, il n'en va pas de même avec celle de Wörthstücke à Sasbach qui s'inscrit parfaitement dans la tradition du Néolithique moyen (site $\mathrm{n}^{\circ} 49$ ). Le défunt a été inhumé allongé sur le dos, les membres inférieurs en extension. Il est orienté selon un axe sud-est/nord-ouest, avec la tête au sud-est. Le mobilier funéraire comprend deux lames en roche polie et les restes d'une jatte décorée. Pour la période qui nous intéresse ici, une dernière tombe est signalée dans le secteur du Kaiserstuhl, celle de Leiselheim (Bade) attribuée au BORS. Malheureusement, la description dont nous disposons se limite à préciser l'orientation du défunt, est-nord-est-ouest-sud-ouest (site $\mathrm{n}^{\circ} 46$ ).

L'esquisse qui se dessine lentement de la transition Néolithique moyen-récent en Alsace et dans le Bade est assez contrastée. Elle montre que si les pratiques funéraires héritées du Néolithique moyen survivent jusqu'à la charnière des $\mathrm{V}^{\mathrm{e}}$ et $\mathrm{IV}^{\mathrm{e}}$ millénaires, elles ne constituent plus la seule norme. L'horizon qui court de la fin du Roessen au début du Michelsberg marque ainsi l'apparition des premières inhumations en fosse circulaire, de la position fléchie et des restes humains en dépôt secondaire. Des différences régionales semblent également se dessiner entre la Basse-Alsace, plus perméable aux nouvelles pratiques, et le sud de la Haute-Alsace, région restée plus tardivement à l'écart des influences occidentales et demeurée fidèle à la tradition du Néolithique moyen.

\section{LE NÉOLITHIQUE RÉCENT (4000-3400 AV. J.-C.)}

Le Néolithique récent voit la généralisation de l’inhumation en fosses circulaires qui représente alors plus des deux tiers du corpus des inhumations connues. Cette période est également marquée par la quasi-disparition des «vraies» tombes dont seuls trois exemplaires sont parvenus jusqu'à nous. Elle se caractérise en outre par un certain opportunisme avec la réutilisation de structures domestiques pour inhumer des corps.

Si on ne tient compte que des inhumations datées par du mobilier ou par le radiocarbone, seules 38 fosses du Néolithique récent ont livré des restes humains, soit une série équivalente à celle de la période précédente. Ce chiffre s'élève à 93 si on retient toutes les fosses dont la datation ne repose que sur le contexte de découverte ou la morphologie des creusements. Pour tenter d'approcher l'évolution des pratiques funéraires au cours des 600 ans sur lesquels s'étire cette période, seuls les ensembles bien datés ont été pris en compte.

En Basse-Alsace, le Michelsberg moyen (III) est de loin l'étape la mieux documentée avec onze inhumations qui peuvent lui être attribuées: dix ont été découvertes dans des fosses circulaires, la dernière dans une "vraie" tombe (Altmatt à Holtzheim, st. 88, site $\mathrm{n}^{\mathrm{o}} 12$ ). L'étape récente du Michelsberg (IV) est moins bien dotée: une seule inhumation en fosse circulaire, une autre multiple dans un complexe de fosses et une tombe (au contournement routier de Marlenheim, site $n^{\circ} 17$ ). Plusieurs autres inhumations datées par radiocarbone peuvent également appartenir au Michelsberg, mais sans que l'on puisse écarter une attribution au Munzingen (fig. 4). Cette culture n'est représentée que par trois inhumations et deux os isolés découverts dans des fosses de type silo (Leimen à Rosheim et Bruechel à Geispolsheim, sites ${ }^{\text {os }} 24$ et 8). Quatre appartiennent à l'étape B du Munzingen et une seule à l'étape C (secteur RMS/rue Ampère à Reichstett, st. 102 , site $\mathrm{n}^{\mathrm{o}} 23$ ).

La situation est plus simple pour la Haute-Alsace et le sud du Bade où seule la culture de Munzingen est représentée au Néolithique récent. Toutes les inhumations qui y ont été découvertes doivent donc lui être attribuées. Toutefois, si on ne considère que celles datées par ${ }^{14} \mathrm{C}$ ou par du mobilier, il ne reste plus que huit ensembles pour essayer de décrire l'évolution des inhumations au cours de cette période. Les plus anciennes sont celles de Lerchenberg à Didenheim (st. 2 et 5 , site $n^{\circ} 31$ ) qui ont livré un mobilier caractéristique de l'étape A, mais pour lesquelles le radiocarbone donne une fourchette plus récente (fig. 5). Si on se fie à la datation des gobelets en bois de cerf sur le plateau Suisse, il faut également conclure que l'inhumation inférieure de Kleinkems date aussi de l'étape A du Munzingen, tout comme la fosse 28 de Didenheim/Morschwiller-le-Bas (sites $n^{\text {os }} 45$ et 33). Pour cette dernière, le radiocarbone donne encore une fois une date plus - ou trop - jeune. L'étape récente du Munzingen (B) est représentée par plusieurs inhumations en fosse de Colmar (st. 41, site $\mathrm{n}^{\circ}$ 30), de Munzingen (st. II/3, site $\mathrm{n}^{\mathrm{o}} 47$ ) et probablement de Didenheim/ Morschwiller-le-Bas (st. 1, site $n^{\circ} 33$ ), quoique de nouveau le ${ }^{14} \mathrm{C}$ ne s'accorde pas avec le mobilier funéraire qui y a été découvert. Sur la base du radiocarbone, il faudrait également ajouter à cette liste la fosse 8 du même site. Le vase 
de l'inhumation supérieure de Kleinkems a également été attribué au Munzingen B, mais il trouve aussi des affinités étroites avec des récipients du site de Lerchernberg à Didenheim (Munzingen A; site $n^{\circ} 31$ ).

\section{L'ENVIRONNEMENT DES INHUMATIONS}

\section{LES SITES}

L'analyse de l'environnement des inhumations ne peut se concevoir que sur les sites ayant bénéficié de décapages suffisamment étendus; or les sites d'habitat du Néolithique récent alsacien ont le plus souvent été appréhendés sur de petites surfaces. Seuls quelques-uns, étudiés récemment, permettent de se faire une idée assez précise de l'organisation spatiale des habitats et, le cas échéant, de la place qu'y occupent les inhumations en fosses circulaires. L'image qui ressort des fouilles récentes est celle d'un habitat plutôt dispersé, caractérisé par de petites concentrations de silos diachrones intégrés dans des cycles d'abandon/ création (Jeunesse, Sainty, 1986) et reliés à des unités domestiques distinctes. Sur la plupart de ces sites des structures de stockage désaffectées réutilisées comme fosses à détritus ont été reconnues, constituant ainsi un indice de la proximité immédiate entre les zones dévolues au stockage et les maisons. Le site de Colmar constitue l'unique exception à ce modèle en Alsace: là, sur une surface très réduite, se concentrent plus de 70 fosses de type silo éloignées des zones d'activités et n'ayant jamais été réutilisées comme fosses de rejets. Elles ont en revanche accueilli treize inhumations primaires, onze dépôts secondaires d'ossements humains et cinq animaux entiers ou partiels. On peut en déduire l'existence d'un secteur "funéraire» bien identifié, peut-être également dévolu à d'autres activités à caractère rituel (dépôts, manipulations) et localisé à distance des habitats. Le cas n'est pas isolé: sur le site du secteur RMS/rue Ampère à Reichstett, F. Blaizot a bien montré que les inhumations Michelsberg se répartissaient en périphérie sud du secteur réservé aux activités domestiques (Blaizot, 2001); sur ce site, toutes les structures riches en artefacts et en dépôts organiques se concentrent au nord d'un paléo-vallon alors que les inhumations sont localisées au sud de ce dernier, dans un secteur où les creusements, répartis selon une trame plus lâche, n'ont livré que de rares tessons erratiques. À Leimen, à Rosheim, les inhumations datées du Michelsberg moyen sont réparties au sein d'un ensemble de silos d'où les rejets détritiques sont totalement absents; l'unique structure riche en mobilier contemporain, localisée lors du diagnostic dans une zone n'ayant pas été prescrite à la fouille, est située à quelque $130 \mathrm{~m}$ au nord-est des inhumations (Lefranc, Boës, 2006).

Dans le deuxième cas de figure, on observe une imbrication étroite entre les inhumations et les secteurs d'activités, imbrications dont témoigne la présence de couches de rejets domestiques dans des fosses utilisées à un moment ou un autre comme fosses sépulcrales. C'est ce qui ressort de l'analyse des ensembles de Lerchenberg à Didenheim ou de la Sablière Maetz à Rosheim, sites dont nous n'avons malheureusement qu'une connaissance très limitée.

Enfin, signalons le cas des inhumations qui apparaissent aujourd'hui isolées, sinon éloignées de tout autre vestige contemporain. Même si, comme à Matzenheim, leur localisation non loin des limites de fouille, interdit de conclure définitivement à leur isolement, cette situation doit être évoquée. Elle a été rencontrée à plusieurs reprises, par exemple sur les sites du lotissement La Peupleraie à Marlenheim ou de la zone 1 de Didenheim/Morschwiller-le-Bas (sites n ${ }^{\text {os }} 19$ et 33). La situation sur le site voisin de la ZAC des Collines à Didenheim est similaire ( site $^{\circ}$ 32). L'inhumation multiple est la seule structure du Néolithique récent découverte sur les 5,5 ha décapés avec une fosse Munzingen à plusieurs centaines de mètres. L'érosion seule ne peut expliquer cet isolement.

\section{LES CREUSEMENTS}

L'une des questions récurrentes est celle de la caractérisation des creusements, soit comme fosses sépulcrales spécialement aménagées pour accueillir le corps, soit comme creusements préexistants dont la fonction sépulcrale est secondaire.

Les fosses sépulcrales «vraies» sont attestées mais demeurent extrêmement rares: nous avons déjà mentionné les sépultures que l'on peut attribuer à l'horizon BruebachOberbergen, BORS et Michelsberg ancien (Sasbach, Eckbolsheim et Matzenheim; sites $\mathrm{n}^{\text {os }} 49,2$ et 20). Pour le Néolithique récent, nous connaissons trois exemples de tombes dont deux, datées par radiocarbone, contenaient des individus allongés sur le dos et orientés sud-sud-est-nordnord-ouest, à Altmatt à Holtzheim (MK III; Lefranc, 2001; site $n^{\circ} 12$ ) et au lotissement La Peupleraie à Marlenheim (MKIII/IV ou MZB; Châtelet, 2002; site $n^{\circ} 19$ ). La tombe du contournement routier à Marlenheim (Tristan, 2004; site $n^{\circ} 17$ ) est l'unique exemple régional où l'on observe un individu fléchi, accompagné d'un vase Michelsberg IV, repo- 
sant dans un creusement ovale ajusté à la taille du corps. Il est orienté nord-ouest/sud-est. De par sa forme et ses dimensions, la fosse de la ZAC des Collines à Didenheim peut être rapprochée de ces fosses sépulcrales (site $n^{\circ} 32$ ). Elle se singularise toutefois par la présence des restes très incomplets de trois enfants dont deux en position secondaire.

Nous avons déjà signalé l'existence au Néolithique récent de la réutilisation de structures domestiques pour inhumer des corps. Cette pratique concerne d'autres types de creusements. On peut citer le dépôt secondaire en chablis des Abattoirs à Holtzheim (Kühnle et al., 1999-2000; site $\mathrm{n}^{\circ} 12$ ), l'inhumation dans une fente partiellement comblée de Hohatzenheim (Lambach, 1986; site $\mathrm{n}^{\circ} 11$ ), les minières du sud de la plaine du Bade et la fosse polylobée de Rosenmeer à Rosheim (site $\mathrm{n}^{\circ}$ 26). Mis à part ces cas anecdotiques, les restes humains apparaissent au Néolithique récent essentiellement dans des fosses de plan circulaire ou subcirculaire généralement assimilées à des fosses-silos (76 cas). Cette assimilation repose sur l'analogie évidente entre les fosses de stockage formant la quasi-totalité des structures exhumées sur les sites du Néolithique récent et les fosses accueillant les inhumations. La question d'une réutilisation opportuniste de structures désaffectées partiellement comblées ou, au contraire, du caractère spécifiquement funéraire de ces creusements, a été amplement débattue lors des deux dernières décennies sans qu'aucun argument irrévocable ne se dégage en faveur de l'une ou l'autre proposition. Les observations récentes réalisées à Leimen, à Rosheim ( site $\mathrm{n}^{\mathrm{o}} 24$ ), à l'Aéroparc à Entzheim (site no 6 ) et à l'Aérodrome à Colmar ( site no 30 ), où il apparaît nettement que les fosses, restées ouvertes, se sont progressivement comblées par ruissellement ou par apport anthropique avant d'être utilisées comme fosses sépulcrales, permettent de valider de façon certaine la première hypothèse ${ }^{4}$. On ne peut cependant exclure théoriquement qu'au sein du corpus régional, certains creusements de plan circulaire accueillant des corps déposés sur le fond aient été spécialement aménagés pour la circonstance.

4. On peut ici proposer un rapprochement avec les restes humains attribués au Michelsberg, découverts dans les remplissages des minières de Spiennes en Wallonie (Toussaint et al., 1997). Mentionnons également pour mémoire les inhumations en grottes et abris dans les zones karstiques de Lorraine (Blouet et al., 1980; Blouet, Guillaume, 1984) et de Belgique (Toussaint, Becker, 1994).

\section{PRATIQUE FUNÉRAIRE ET TRAITEMENT DES CORPS}

\section{POSITION ET ORIENTATION DES CORPS}

La position considérée comme conventionnelle au Néolithique récent est la position contractée sur le flanc, membres inférieurs repliés, le plus souvent vers la poitrine. Cette position caractérise en effet l'immense majorité des sépultures du domaine d'étude, du Chasséen au sud (Beeching, Crubézy, 1998) au groupe de Baalberg à l'est (Preuss, 1966).

Au sein du corpus étudié, la position des membres supérieurs accuse une grande variabilité; ils sont fréquemment ramenés vers le visage, plus rarement sur les épaules ou sur le pubis. Mentionnons, à titre de comparaison au sein d'un abondant corpus, les exemples de Leimen à Rosheim 94 (fig. 6) et 104-1 (site $\mathrm{n}^{\mathrm{o}}$ 24), Entzheim/Geispolsheim, st. 1467 (site $\mathrm{n}^{\mathrm{o}}$ 6), Didenheim/Morschwiller-le-Bas st. 28 (individu 3 ; site $\mathrm{n}^{\circ} 33$ ) ou encore Altmatt à Holtzheim, st. 260 (site $\mathrm{n}^{\mathrm{o}} 12$ ). Une minorité de défunts est couchée sur le dos, genoux ramenés sur le thorax à l'instar des individus 154 et 104/2 de Leimen à Rosheim et Didenheim/ Morschwiller-le-Bas, st. 1 (individu 1). Il ne s'agit là que d'une variante de la position contractée sur le flanc.

Les individus inhumés sur le dos, membres inférieurs en extension, qui perpétuent la tradition funéraire du Néolithique moyen régional - tradition attestée jusqu'à la charnière des $\mathrm{V}^{\mathrm{e}}$ et $\mathrm{IV}^{\mathrm{e}}$ millénaires (Lefranc et al., 2009) - pourraient constituer une seconde catégorie au sein des positions «conventionnelles». Ils ne sont jusqu'ici connus que par quatre individus inhumés sur les sites de Matzenheim (site $n^{\circ} 20$; fig. 7), d'Am Schluesselberg et d'Altmatt à Holtzheim (site $\mathrm{n}^{\mathrm{o}} 12$; fig. 8) et de Marlenheim (site $\mathrm{n}^{\circ} 19$ ), respectivement datés du Michelsberg ancien, du Michelsberg moyen et du Michelsberg ou du Munzingen.

La seconde catégorie rassemble les individus en position «désordonnée» (Jeunesse, 2010) ou «inorganisée» (Blaizot, 2001). Il est assez difficile de proposer un terme satisfaisant susceptible de rendre compte de la grande variabilité des positions rencontrées dont le seul dénominateur commun réside dans leur éloignement par rapport à la position définie comme «conventionnelle» ou «régulière». Que ces positions variées traduisent des gestes funéraires complexes ou, au contraire, l'absence de tels gestes - question qui nous semble aujourd'hui très difficile à trancher -, elles amènent à s'interroger sur le statut de ces individus bénéficiant d'un mode d'inhumation en rupture avec la 


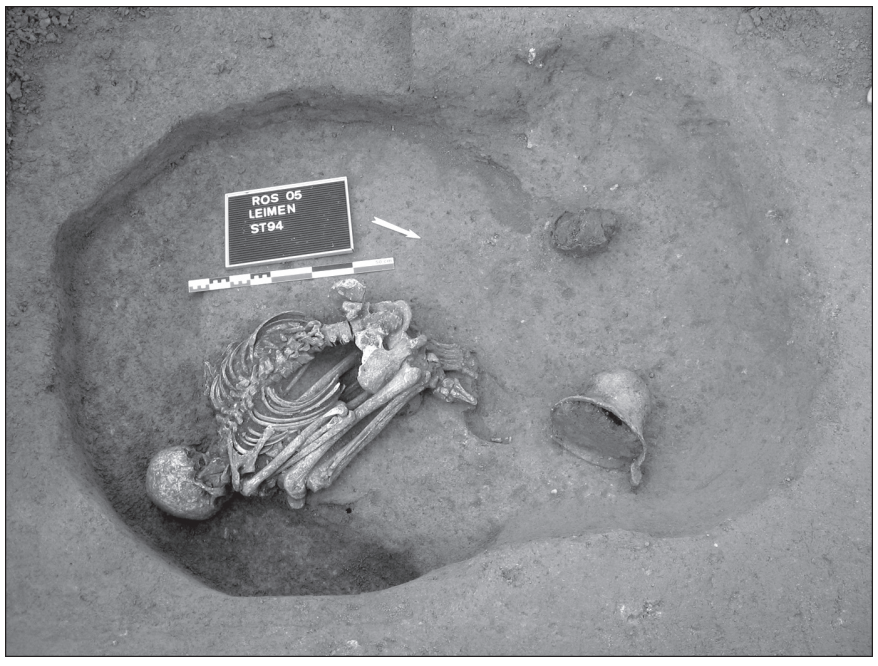

Fig. 6 - Leimen à Rosheim, inhumation st. 94 (cliché: Ph. Lefranc, Inrap).

norme. Nous reviendrons plus longuement sur cette question complexe en abordant l'analyse des dépôts simultanés.

La gamme des orientations couvre un large éventail (fig. 9). Nous n'analyserons ici que les inhumations «régulières»; en effet, pour les individus en position «désordonnée» n'ayant peut-être pas bénéficié de gestes funéraires, l'orientation peut être aléatoire ou, dans le cas des inhumations multiples, définie en opposition à l'orientation de l'individu principal.

En Basse-Alsace, il est bien difficile de distinguer une orientation majoritaire: les orientations s'inscrivant dans les quadrants sud-est, nord-ouest et nord-est apparaissent dans des proportions équivalentes. Seule la portion sudsud-ouest est délaissée (une unique occurrence). Rappelons qu'il y a peu de temps encore, avant la publication des sites RMS/rue Ampère à Reichstett, d'Entzheim-Geispolsheim ou de Leimen à Rosheim, l'orientation tête au sud-est pouvait être considérée comme majoritaire pour l'ensemble du domaine Michelsberg (Nickel, 1998), image fortement bouleversée par les données récentes.

En Haute-Alsace, la situation est différente mais il faut souligner que le corpus est beaucoup plus réduit qu'en Basse-Alsace, ce qui est susceptible d'introduire une importante distorsion: mentionnons également le fait que dans certaines publications relativement anciennes (Schweitzer, 1987; Chatton, 1974 et 1976) qui constituent une grande part du corpus, les orientations ne sont pas toujours clairement spécifiées et toujours difficiles à vérifier. Si l'on se réfère à notre figure, il apparaît que les orientations privilégiées se rapprochent du nord et de l'est. On note

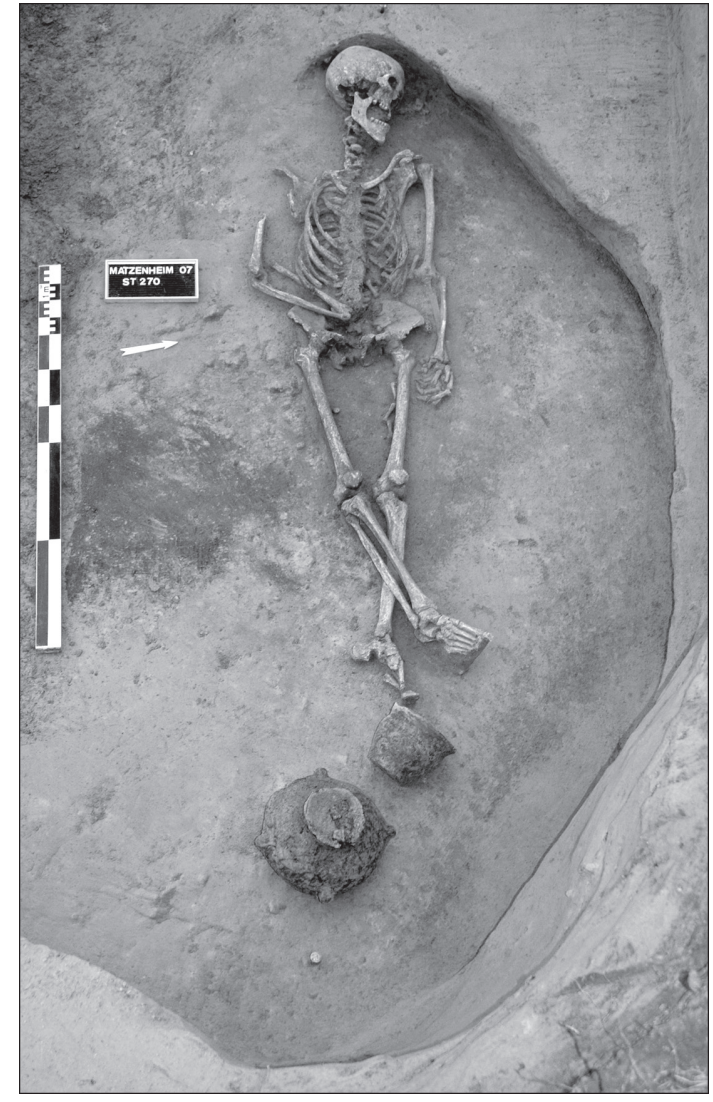

Fig. 7 - Le Lavoir à Matzenheim, vue de la tombe Michelsberg ancien du Rhin supérieur (d'après Toullec, 2008) (cliché: C. Toullec, Antea-Archéologie).

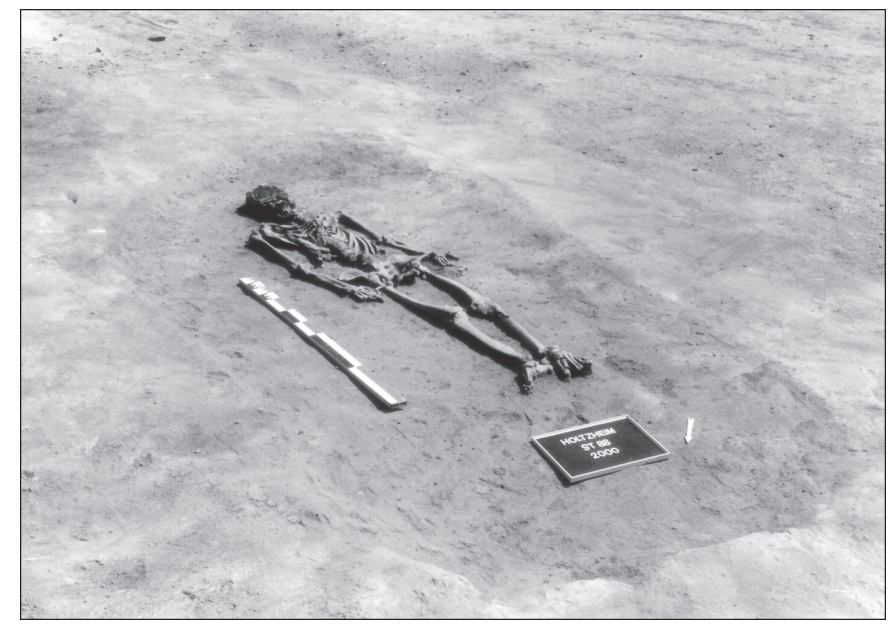

Fig. 8 - Altmatt à Holtzheim, inhumation st. 88, individu allongé sur le dos dans un creusement quadrangulaire (cliché: Ph. Lefranc, Inrap). 


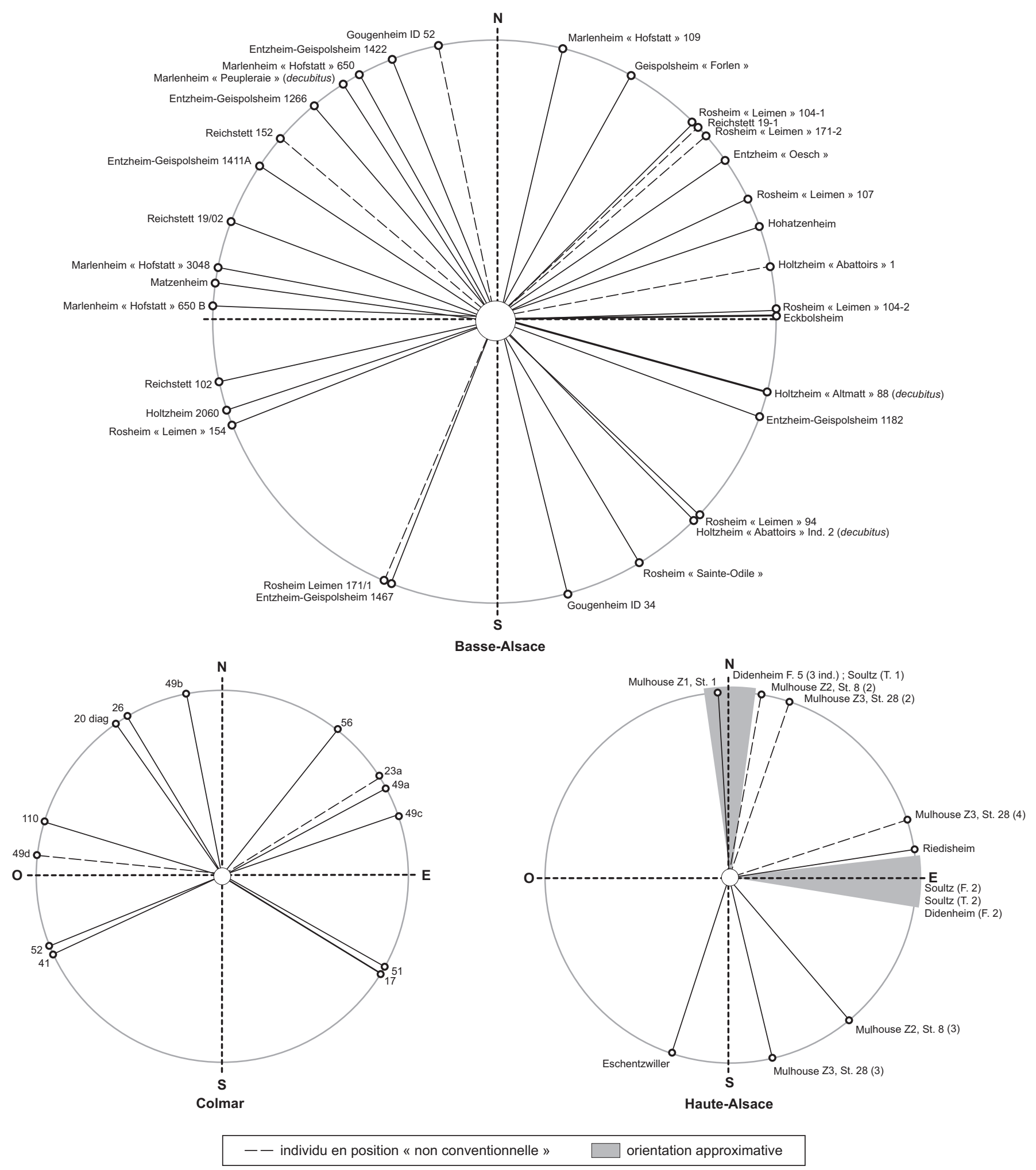

Fig. 9 - Orientations des individus en Basse- et Haute-Alsace et dans la région de Colmar (Haut-Rhin)

(DAO: Ph. Lefranc, Inrap). 
également une orientation au sud-est, ainsi que deux individus se rapprochant de l'axe nord-sud (sud/sud-est et sud/ sud-ouest).

L'absence d'inhumations orientées dans un quadrant nord-ouest ou ouest/sud-ouest, bien attestées en BasseAlsace, pourrait identifier un clivage entre les deux régions.

Sur le site de Colmar, localisé en territoire Munzingen, légèrement au sud du secteur marquant la frontière entre les groupes de Haute- et de Basse-Alsace, l'orientation la mieux représentée avec quatre occurrences est l'orientation dans le quart nord-ouest; suivent l'orientation est/ nord-est, puis les orientations est/sud-est et ouest/sud-ouest (site $n^{\circ} 30$ ). Le fait que les orientations évoquent davantage les inhumations de Basse-Alsace ne constitue en rien un obstacle: on sait que les frontières des provinces funéraires ne correspondent que rarement à celles définies par les styles céramiques (Jeunesse, 2004).

\section{COMPOSITION DES DÉPÔTS}

Les 95 structures du Néolithique récent ont livré les restes de plus de 150 individus, dont une majorité inhumés entiers ou subcomplets. On dénombre également sept corps largement incomplets ou parties de cadavres en connexion anatomique ainsi que 23 os isolés. En ce qui concerne uniquement les 76 fosses circulaires, elles rassemblent plus de 124 individus dont 100 complets ou presque ${ }^{5}, 18$ os isolés et 6 squelettes incomplets ou parties de squelettes.

Sauf dans la région de Mulhouse où les inhumations plurielles sont plus fréquentes, les dépôts, que ce soit en fosses circulaires, dans des tombes ou dans tout autre type de creusement, concernent dans deux tiers des cas des individus seuls. Sur les 30 inhumations plurielles recensées, les cas les plus fréquents sont ceux réunissant les restes de deux ou trois individus (respectivement 13 et 11 occurrences). On compte également cinq inhumations quadruples et une rassemblant 8 corps (Bollwiller, site $\left.n^{\circ} 29\right)$. Le cas de figure le plus fréquent est celui d'individus entiers ou subcomplets (22 occurrences), plus rarement inhumés avec des squelettes incomplets ou des os isolés, généralement des crânes (3 occurrences respectives).

5. Ce cas concerne les inhumations mises au jour lors de fouilles anciennes. Les squelettes semblent complets mis à part un ou quelques petits ossements, mais sans qu'il soit possible de déterminer si nous avons affaire à un prélèvement volontaire, aux aléas de la taphonomie ou, plus probablement, aux lacunes de fouilles souvent rapides et menées par un personnel non qualifié.

\section{LES INHUMATIONS PLURIELLES : DÉPÔTS SUCCESSIFS ET DÉPÔTS SIMULTANÉS}

En Basse-Alsace, 13 inhumations plurielles ont été fouillées. Il s'agit, à une exception près, de fosses circulaires. Elles ont livré les restes de 2 à 4 individus :

- Tuilerie Schaefer à Achenheim: 2 adultes (site $n^{\circ} 1$ ) ;

- Aéroparc d'Entzheim/Geispolsheim, st. 1266: 2 adultes (site $\left.\mathrm{n}^{\circ} 6\right)$;

- Aéroparc d'Entzheim/Geispolsheim st. 1411: 1 adulte et 1 enfant;

- Am Schluesselberg à Holtzheim, st. 79: 2 adultes et 1 bloc craniofacial (site $\left.n^{\circ} 12\right)$;

- Sablière Lienhardt à Lingolsheim, st. 39: 2 adultes (site $\left.\mathrm{n}^{\circ} 16\right)$;

- Hofstatt à Marlenheim, st. 109: 1 adulte, 2 enfants et 1 individu en positon secondaire ou remanié (site $\left.n^{\circ} 18\right)$;

- Hofstatt à Marlenheim, st. 650: 1 adulte en contact avec un bloc craniofacial;

- Hausbergen à Mundolsheim: 3 enfants (site $n^{\circ} 22$ );

- RMS/rue Ampère à Reichstett, st. 19: 2 adultes (site no 23) ;

- Leimen à Rosheim, st. 104: 2 adultes et 1 périnatal (site $\left.\mathrm{n}^{\circ} 24\right)$;

- Leimen à Rosheim, st. 171: 1 adulte et 1 enfant;

- Rosenmeer à Rosheim, st. 31: 3 adultes et 1 enfant (site $\left.\mathrm{n}^{\circ} 26\right)$;

- Sablière Maetz à Rosheim, st. 7 : 2 adultes (site no 27).

$\mathrm{Si}$ on considère la Haute-Alsace dans son ensemble, un tiers des inhumations de cette période sont plurielles (12 fosses). Cette proportion varie fortement entre le nord de la région où elle ne dépasse pas $20 \%$ et la région mulhousienne où elle atteint $70 \%$. Comme pour la BasseAlsace, les creusements sont circulaires, à l'exception de la fosse de la ZAC des Collines à Didenheim de plan ovale. Le nombre d'individus varie de 2 à 8 :

- Bollwiller: 7 adultes et 1 enfant (site no 29);

- Aérodrome de Colmar, st. 49: 2 adultes et 2 enfants (site $\left.\mathrm{n}^{\circ} 30\right)$;

- Lerchenberg à Didenheim, fosse 5: 3 enfants (site $n^{\circ} 31$ ) ;

- Lerchenberg à Didenheim, fosse 13: 1 adulte et 2 enfants;

- ZAC des Collines à Didenheim: 3 enfants (site no 32) ;

- Rocade ouest à Didenheim/Morschwiller-le-Bas, zone 1, st. 1 : 1 adulte et 1 enfant (site $n^{\circ} 33$ );

- Rocade ouest à Didenheim/Morschwiller-le-Bas, zone 2, st. 8: 1 adulte et 3 enfants;

- Rocade ouest à Didenheim/Morschwiller-le-Bas, zone 3, st. 28: 2 adultes et 2 enfants;

- Katzenthal: 3 enfants (site $n^{\circ} 35$ ); 
- Rue des Violettes à Riedisheim: 1 adulte et 2 enfants (site $\left.\mathrm{n}^{\circ} 38\right)$;

- Buhlfeld à Soultz, F1: 1 adulte et 3 enfants (site no 39);

- Koenigsbreite à Wettolsheim: 2 individus (site $\mathrm{n}^{\mathrm{o}} 41$ ).

Dans le sud du Bade, si on laisse de côté les inhumations en minière, une seule inhumation plurielle y est connue, celle de Jechtingen (site $\mathrm{n}^{\mathrm{o}} 43$ ) qui a livré le corps d'un adulte et les restes fragmentés d'un second individu.

On l'aura noté, l'association adulte/enfant est très fréquente: 13 occurrences, soit la moitié du corpus. Elle est même systématique en Haute-Alsace où nous ne connaissons aucun exemple d'inhumation plurielle ayant exclusivement livré des individus adultes. Ces dernières, toutes localisées en Basse-Alsace, sont au nombre de cinq (RMS/rue Ampère à Reichstett, Sablière Maetz à Rosheim, Achenheim, Am Schluesselberg à Holtzheim, Entzheim st. 1266; sites $\mathrm{n}^{\mathrm{os}} 1$, 6, 12, 23 et 27). Quatre structures contenaient uniquement des enfants (Dorfburg à Katzenthal, Lerchenberg fosse 5 et ZAC des Collines à Didenheim et Hausbergen à Mundolsheim; sites $\mathrm{n}^{\text {os }} 22,31,32$ et 35).

La plupart des inhumations sont des inhumations primaires mais plusieurs sujets ont été déposés dans un état de décomposition déjà avancée, voire ne sont représentés que par des fragments, notamment des crânes. Ces dépôts secondaires sont signalés à la ZAC des Collines à Didenheim, la Rocade ouest à Didenheim/Morschwiller-le-Bas, rue des Violettes à Riedisheim, Hofstatt à Marlenheim (st. 109 et 650) et Am Schluesselberg à Holtzheim (sites $n^{\text {os }} 12,18$, 32,33 et 38 ).

Les études récemment menées par les anthropologues de terrain ont certes permis de démontrer que certains corps se sont décomposés dans des espaces vides mais il est souvent difficile de déterminer si le maintien d'un tel espace s'étend réellement à l'ensemble de la fosse ou uniquement à l'espace occupé par le cadavre: l'hypothèse d'un élément semi-rigide en matière périssable recouvrant le corps a plusieurs fois été émise (Blaizot, 2001 ; Lefranc et al., 2007). L'existence d'un vide étendu à l'ensemble de la fosse peut cependant être retenue dans certains cas, observation qui revêt une importance toute particulière pour les interprétations que l'on peut proposer pour les inhumations plurielles ou les déplacements d'ossements observés sur certains squelettes. Nous nous appuierons essentiellement, pour cette présentation, sur les ensembles récemment fouillés et ayant bénéficié de l'intervention in situ d'un anthropologue, notamment sur les sites de Leimen à Rosheim, de l'Aérodrome à Colmar et de Hofstatt à Marlenheim (sites $\mathrm{n}^{\mathrm{os}} 18$, 24 et 30$)$.

\section{LES DÉPÔTS SUCCESSIFS}

En Alsace, une sépulture plurielle témoignant de dépôts successifs au sein d'un espace demeuré accessible a récemment été étudiée par E. Boës sur le site de Leimen à Rosheim (site $\mathrm{n}^{\circ}$ 24). La fosse 104 de ce site contenait les restes de trois individus dont deux adultes et un nourrisson, reposant apparemment sur un même niveau mais n'ayant pas bénéficié des mêmes conditions de décomposition (fig. 10) (Lefranc et al., 2007). Le jeune individu de sexe masculin âgé de 16 à 18 ans (104/2), déposé au centre de la fosse en position conventionnelle, a bénéficié d'un colmatage différé comme le suggèrent certains déplacements d'ossements observés en particulier au niveau de la mandibule, du rachis cervical et des pieds. Le maintien de certaines connexions instables pourrait être imputé au recouvrement du corps par un matériau souple. L'hypothèse d'une décomposition en espace vide ne peut pas être écartée. Un nourrisson (individu 104/3) reposait à son contact. La femme (104/1) a été déposée contre la paroi sud du creusement, également en position conventionnelle: aucun os n'ayant été retrouvé en dehors du volume corporel initial, l'étude taphonomique conclut à un colmatage immédiat du corps. L'hypothèse d'un dépôt successif des corps permet d'expliquer, outre les dissemblances mentionnées, les différences d'altitudes notées entre le crâne de l'individu en position centrale et la main de l'individu 104/1, localisée quelques centimètres plus haut.

Cet unique exemple pourrait signaler un mode de fonctionnement collectif se rapprochant, comme l'a souligné

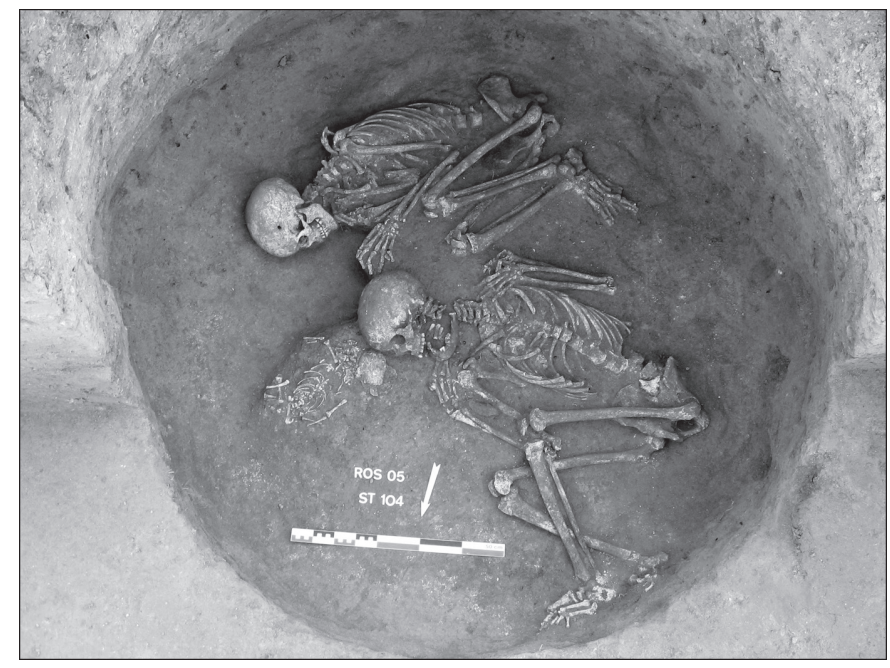

Fig. 10 - Leimen à Rosheim, vue de la sépulture multiple st. 104 (cliché: Ph. Lefranc, Inrap). 
C. Jeunesse, de celui des sépultures contemporaines en tombe à couloir de l'ouest de la France (Jeunesse, 2010). Le même parallèle avec les chambres mégalithiques de la façade atlantique a été établi par A. Beeching et E. Crubezy à propos de certaines sépultures chasséennes de Saint-Paul-Trois-Châteaux et de Montélimar/Chateauneuf-du-Rhône (Drôme) (Beeching, Crubezy, 1998). Récemment, le caractère collectif de plusieurs ensembles a été retenu par les fouilleurs du site chasséen du Crès à Béziers (Loison et al., 2003).

Les sépultures «à étages» de la rue des Violettes à Riedisheim, de la Sablière Maetz à Rosheim, d'Am Schluesselberg à Holtzheim, de l'Aérodrome à Colmar (fig. 10), de Hofstatt à Marlenheim/st. 650 (sites nos 12, 18, 27, 30 et 38), caractérisées par les dépôts successifs de corps séparés par des couches de sédiment plus ou moins épaisses, pourraient également transcrire un mode de fonctionnement collectif.

\section{DÉPÔTS SIMULTANÉS}

Une autre avancée réside dans l'analyse renouvelée des dépôts multiples où figure un individu "principal» en position conventionnelle, accompagné d'un ou plusieurs sujets en position désordonnée. S'appuyant sur plusieurs exemples chasséens, E. Crubézy avait, en 1991, prudemment avancé l'hypothèse d'une «différenciation sociale horizontale et/ou verticale»(Crubézy, 1991); les publications les plus récentes retiennent très volontiers, pour ces sépultures "asymétriques» de la province funéraire des fosses rondes, le modèle de l'accompagnement (Gallay, 2006; Jeunesse, 2010 ; Boulestin, 2008).

La sépulture multiple de la zone 3 st. 28 de Didenheim/ Morschwiller-le-Bas (site $n^{\circ} 33$ ) peut être rattachée à cette pratique. Elle a livré les corps de deux adultes et de deux enfants (fig. 11 et fig. 12, C et D). Un des adultes a été enterré en position désordonnée à une dizaine de centimètres au-dessus du fond de la fosse (fig. 11, individu 4). Le second adulte a été placé tête-bêche sur le premier, cette fois en position repliée, avec les mains ramenées devant le visage (individu 3). Il est lui-même recouvert par l'un des enfants dans une position également désordonnée (individu 2). Le second enfant a, quant à lui, été déposé au même niveau que les autres corps, mais à côté (individu 1). La topographie générale du squelette n'est pas respectée, mais la représentation osseuse semble satisfaisante. Le squelette a cependant conservé une certaine logique anatomique, ce qui plaide en faveur d'une décomposition au sein d'un contenant (Denaire, 2007).
Les autres exemples régionaux sont moins spectaculaires mais, si l'on accepte la proposition selon laquelle une asymétrie marquée dans le positionnement des corps peut refléter la pratique de l'accompagnement (Testart, 2004), il n'est pas illégitime de proposer d'y assimiler cinq autres dépôts simultanés: nous retiendrons comme probables témoins de cette pratique, et en nous limitant aux cas suffisamment documentés, les ensembles st. 109 de Marlenheim, st. 2/ zone 8 de Didenheim, sépulture 19 de RMS/rue Ampère à Reichstett, st. 49 de l'Aérodrome à Colmar et F1 de Soultz (sites $\mathrm{n}^{\text {os }}$ 18, 23, 30, 33 et 39).

À Marlenheim (site $n^{\circ} 18$ ), figurent trois individus en contact strict dont un adulte inhumé en position conventionnelle contractée et deux enfants en position désordonnée; le dépôt funéraire, constitué d'un grand fragment de gobelet brisé en deux parties, est - à l'instar de ce que l'on observe à Didenheim - clairement associé à l'individu fléchi, un des fragments ayant été directement déposé sur ce dernier.

La fosse 2/zone 8 de Didenheim/Morschwiller-le-Bas contenait les restes de quatre individus répartis sur deux niveaux: sur le fond du creusement étaient disposés deux enfants dont l'un en position fléchie et l'autre en position désordonnée (fig. 12, A et B; site no 33).

Le dépôt inférieur de la «sépulture à étage» de l'Aérodrome à Colmar, déjà citée, est constitué par un adulte reposant en position hypercontractée et un enfant déposé en appui partiel contre la paroi, un membre inférieur en extension, l'autre fléchi au niveau du genou (fig. 13, inhumations C et D; site $n^{\circ} 30$ ).

Les restes de quatre individus (un adulte et trois enfants) ont été découverts dans la fosse 1 de Soultz-HautRhin (site $n^{\circ} 39$ ). D’après les photographies prises lors de la fouille, l'adulte semble placé en position fléchie au centre de la fosse, tandis que les enfants ont été retrouvés contre la paroi de la fosse, en face de l'adulte, les pieds du plus âgé «accolés à la face latérale gauche du crâne de l'adulte » (Chatton, 1976).

Enfin, sur le site RMS/rue Ampère à Reichstett, la fosse 19 a livré deux individus adultes inhumés simultanément, l'un en position fléchie sur le flanc, l'autre en positon désordonnée (site no 23).

Dans tous les cas retenus, le principe d'asymétrie s'applique aux traitements des corps, en position arrangée ou non, ainsi qu'aux orientations toujours divergentes.

Nous n'insisterons pas davantage sur la thèse de l'accompagnement déjà abordée en détail par Jeunesse (2010) et par Gallay (2006) notamment, et qui peut être proposée 


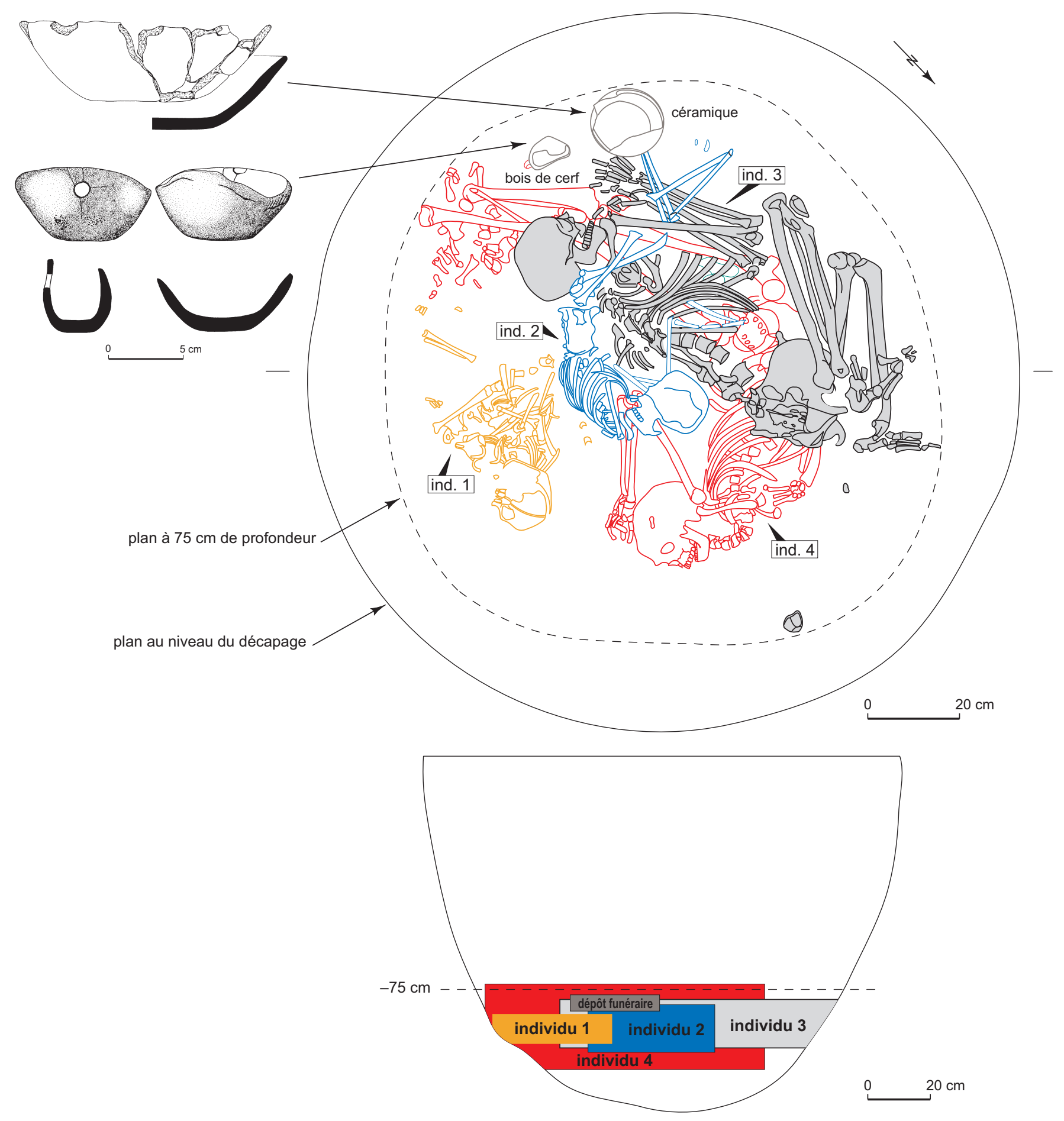

Fig. 11 - La Rocade ouest à Didenheim/Morschwiller-le-Bas, l'inhumation 28 et son mobilier (d'après A. Denaire, 2007). 

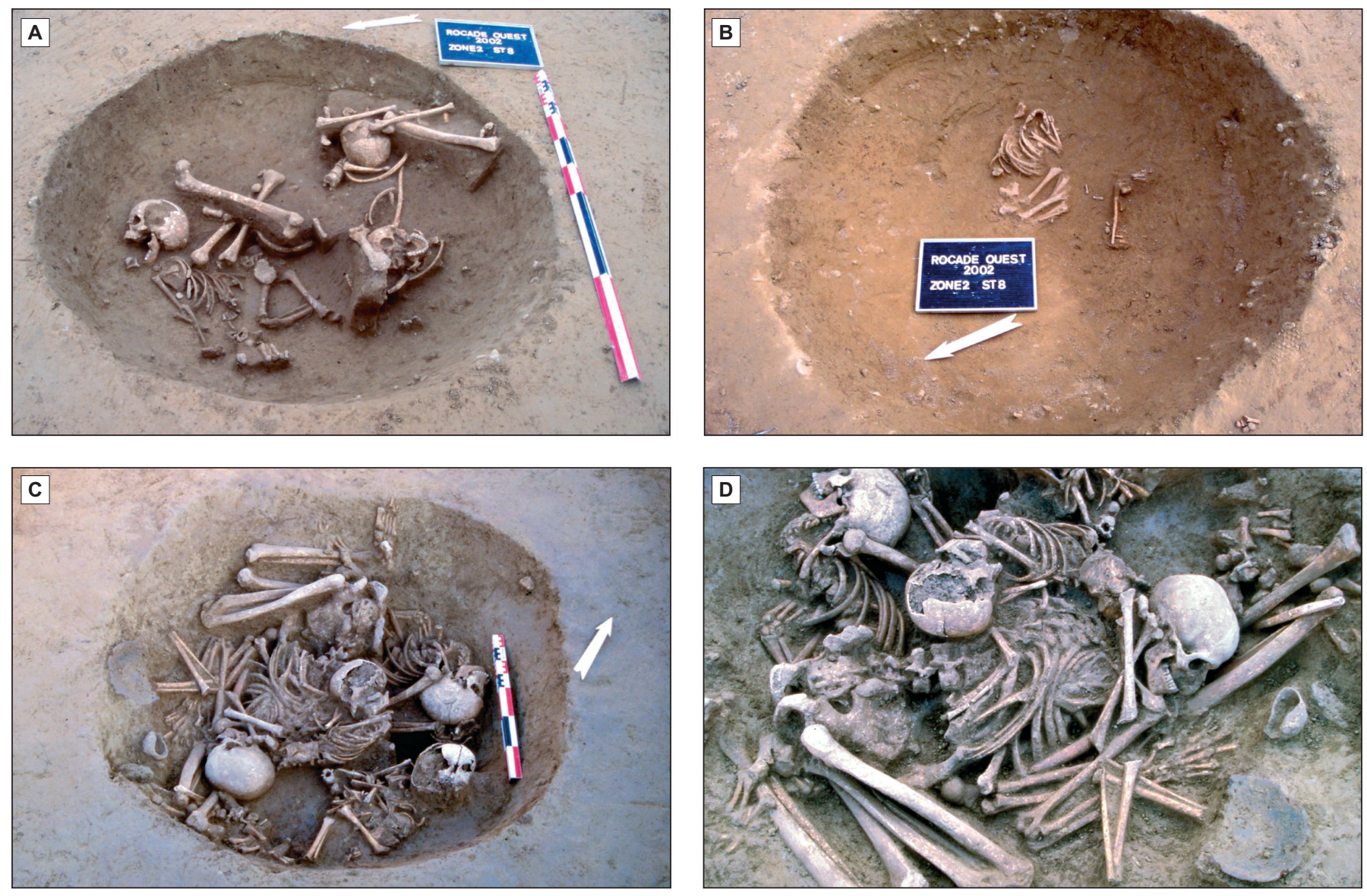

Fig. 12 - La Rocade ouest à Didenheim/Morschwiller-le-Bas, vue des inhumations 8 et 28:A, st. 8, vue du premier décapage (individus 1a, 1 lb et 2); $\boldsymbol{B}$, st. 8, vue du second décapage (individu 3); $\boldsymbol{C}$, st. 28, vue générale; $\boldsymbol{D}$, st. 28, vue rapprochée de l'individu 3 et du mobilier (clichés: Antea-Archéologie).

comme explication. Dans ce cas, l'intérêt de l'ensemble fosse 2/zone 8 de Didenheim/Morschwiller-le-Bas mérite d'être souligné car il montrerait que les adultes ne sont pas seuls à bénéficier de cette pratique. Un autre cas d'enfant en position "principale» est connu sur le site d'Aue à Bruchsal (Nickel, 1998). Cette caractéristique va, comme la pratique de l'accompagnement elle-même (Gallay, 2006), dans le sens d'un degré d'intégration sociale élevé (Lichardus, 1991).

Toutes les sépultures plurielles régionales ne procèdent certainement pas de la pratique de l'accompagnement: certains dépôts simultanés rassemblant trois enfants (Dorfburg à Katzenthal, Lerchenberg à Didenheim, fosse 5 et Hausbergen à Mundolsheim; sites $\mathrm{n}^{\text {os }} 22,31$ et 35), montrent des individus reposant tous en position conventionnelle et respectant la même orientation. En aucun cas, on ne peut évoquer une quelconque hiérarchie entre les individus. Même constat pour les jeunes enfants inhumés au contact d'adultes dans la fosse 104 de Leimen à Rosheim (premier dépôt; site $\mathrm{n}^{\mathrm{o}}$ 24), la fosse 1411 d'EntzheimGeispolsheim (site $\mathrm{n}^{\circ}$ 6) et la fosse 13 de Lerchenberg à Didenheim (site $\mathrm{n}^{\mathrm{o}} 31$ ). Ces sépultures multiples pourraient être reliées à des périodes de crises (épidémies, violences, etc.) affectant particulièrement les individus les plus faibles sans qu'il soit nécessaire d'invoquer la pratique de l'accompagnement (Boulestin, 2008).

\section{DÉPÔTS SECONDAIRES}

Affirmer que les ossements humains ont fréquemment été manipulés au cours du Néolithique récent relève du lieu commun. Les ossements isolés et les segments anatomiques (Teilbestatungen) retrouvés, parfois en masse, en position secondaire dans les remplissages des fossés d'enceinte Michelsberg (Koch, 1971; Charpentier, 1989; Biel, 1990; Behrends, 1998; Perrin, 2006) et les fosses de stockage, témoignent suffisam- 

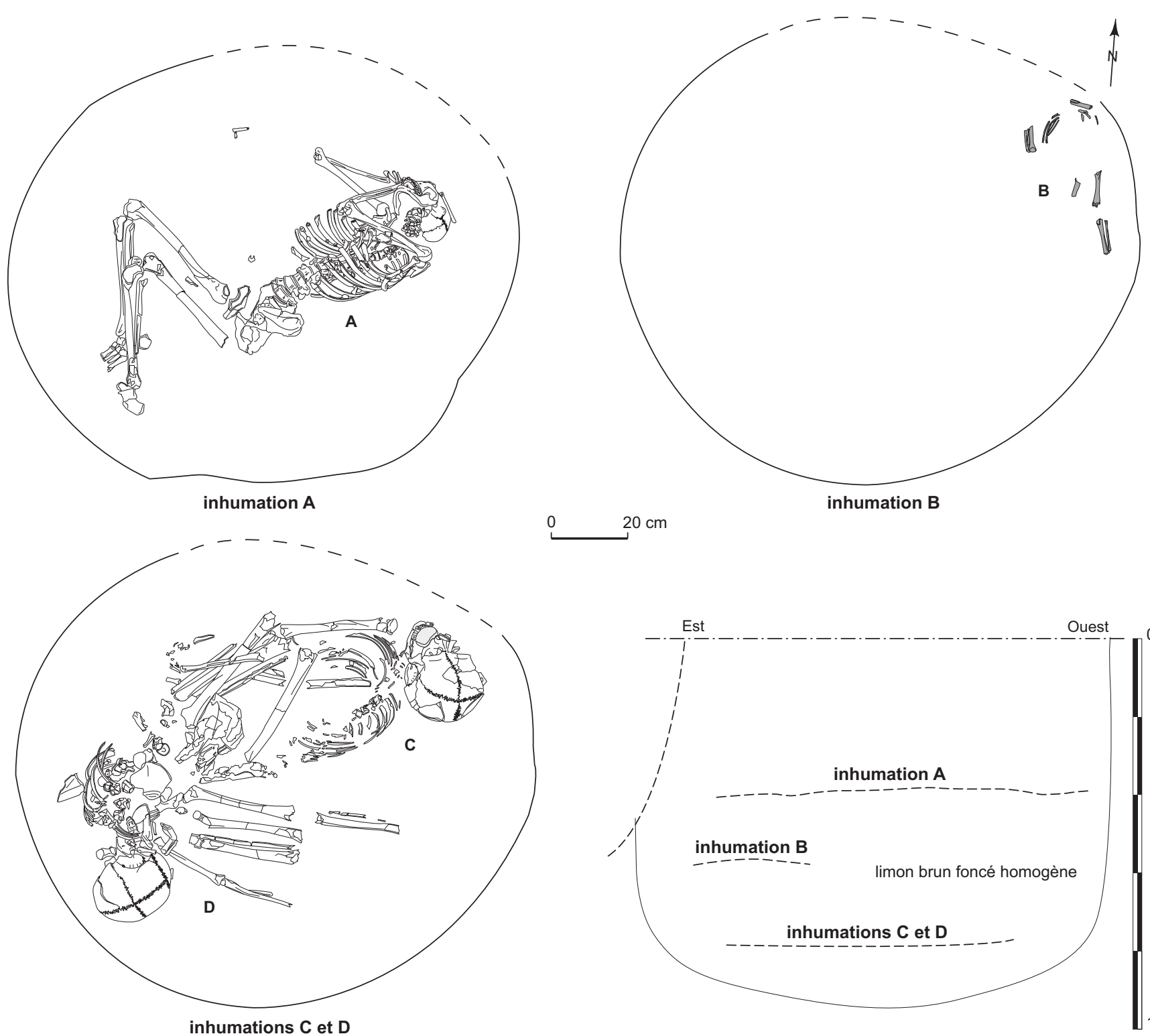

inhumation B

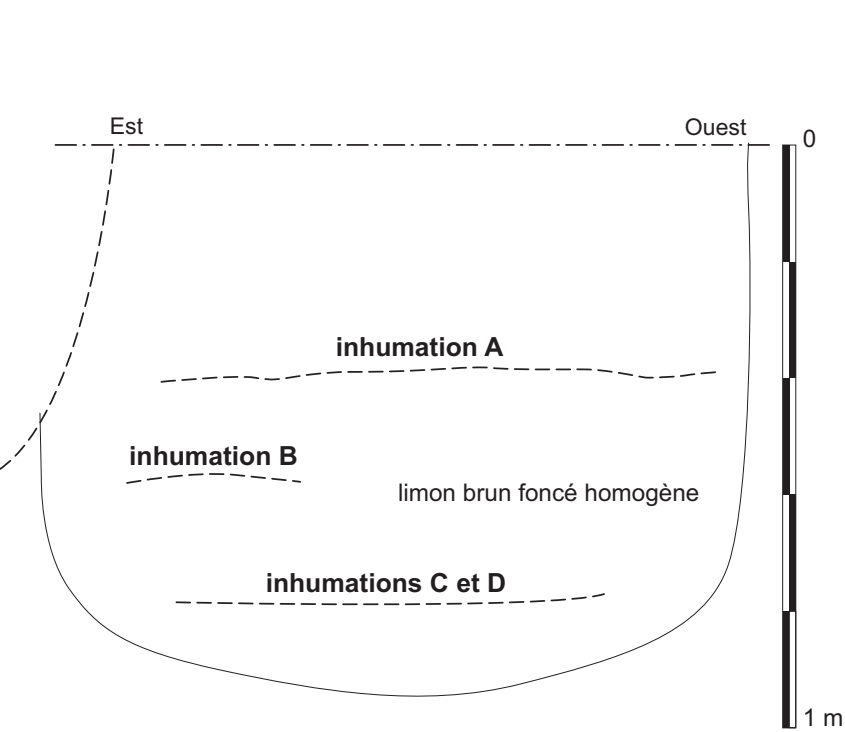

Fig. 13 - L'Aérodrome à Colmar, les trois niveaux d'inhumation de la structure 49 (DAO: Ch. Leyenberger, Inrap).

ment de la place éminente qu'occupe cette pratique dans la gestuelle mortuaire. On peut noter, avec C. Nickel (1998) que la moitié des individus constituant le corpus étudié, n'a pas bénéficié d'un dépôt primaire définitif.

Encore peu connus en Alsace, les ossements isolés sont fréquemment signalés sur les sites Michelsberg des régions voisines; le site de Michelsberg à Untergrombach figure parmi les plus riches en découvertes de ce type avec 172 ossements isolés, répartis dans une vingtaine de fosses et appartenant à un minimum de 34 individus
(Bonnet, 1899; Nickel, 1998). Les explications généralement avancées dans la littérature vont de la manipulation intentionnelle au remaniement involontaire de sépulture. Les fouilles récentes de Didenheim et de Colmar ont livré quelques éléments déterminants permettant de renforcer l'hypothèse de la manipulation intentionnelle (sites $n^{\text {os }} 30$ et 32).

En Alsace et dans le sud du Bade, des ossements en position secondaire sont aujourd'hui attestés dans 20 structures circulaires, une fosse ovale, un chablis, un fossé 
et une minière. On les rencontre quatre fois seulement en contexte détritique, dans trois fosses Munzingen respectivement fouillées à Leimen à Rosheim (site $\left.n^{\circ} 24\right)$, à Bruechel à Geispolsheim (site $\mathrm{n}^{\circ}$ 8) et à Munzingen (st. 49; site $n^{\circ} 47$ ), ainsi que dans une fosse Michelsberg à l'Aéroparc d'Entzheim (site $n^{\circ} 6$ ).

Quatre dépôts secondaires intentionnels ont été retrouvés dans des creusements accueillant également les inhumations d'individus en position primaire: c'est le cas des ossements des deux individus adultes retrouvés dans la partie supérieure du comblement de la fosse st. 8/zone 2 de Didenheim/Morschwiller-le-Bas déjà évoquée (site $n^{\circ} 33$ ), du bloc craniofacial d'Am Schluesselberg à Holtzheim (site $n^{\circ} 12$ ) et des crânes issus des structures 109 et 650 de Hofstatt à Marlenheim (site $\mathrm{n}^{\circ} 18$ ) ainsi que la fosse récemment fouillée à la ZAC des Collines à Didenheim et la structure 51 de Colmar (sites n 30 et 32). Il faudrait éventuellement compléter cette liste avec les fosses de Bollwiller et de Jechtingen, mais la documentation manque pour être formel (sites n 29 et 43).

Le site de l'Aérodrome à Colmar, site particulier n'ayant livré aucun mobilier d'origine détritique, est aujourd'hui l'ensemble régional qui a livré le nombre le plus important de dépôts secondaires (11 structures; site $n^{\circ} 30$ ): ils apparaissent huit fois dans des fosses où ils constituent le seul matériel osseux, deux fois dans des fosses recelant également des dépôts primaires et, enfin, au même niveau qu'un squelette de cervidé. Il est souvent difficile de trancher entre l'hypothèse d'un dépôt ou d'une présence fortuite dans le cas des ossements isolés; deux dépôts seulement (une dizaine d'ossements reposant sur un même niveau et un bloc craniofacial associé à un pendentif) peuvent être identifiés avec certitude à des dépôts volontaires.

\section{MANIPULATIONS POSTDÉCOMPOSITIONNELLES}

La possibilité d'une manipulation in situ d'individus s'étant décomposés dans des espaces non colmatés a été clairement énoncée à propos de la fosse 8/zone 2 de Didenheim/Morschwiller-le-Bas (Jeunesse, 2010). Les deux enfants reposant sur le fond de cette fosse ont fait l'objet d'une intervention postérieure à la disparition des chairs comme l'indiquent le prélèvement du crâne de l'individu en position contractée (fig. 13, B) et les déplacements d'ossements observés sur le sujet en position désordonnée (Denaire, 2007).

À l'Aérodrome à Colmar, deux cas de réintervention sur des corps s'étant décomposés dans des fosses demeurées accessibles ont récemment pu être mis en évidence par l'étude taphonomique (Lefranc, Chenal, 2010).

Pour l'enfant de la fosse 110 de ce site, les indices permettent de conclure à une décomposition en espace vide; le maintien en connexion de certaines articulations labiles autorise en outre à affirmer qu'il s'agit bien là d'un dépôt primaire. Le bloc craniofacial de cet enfant a été retrouvé à $80 \mathrm{~cm}$ au sud du corps et à peu près au même niveau que celui-ci (fig. 14, A). La position de la mandibule, demeurée en place en amont des vertèbres cervicales, et la présence d'une première incisive supérieure au niveau de cette dernière, permettent d'appréhender le moment du déplacement du crâne, intervenu après la décomposition complète des chairs. Cette manipulation, qui n'a pas perturbé le reste du squelette demeuré en connexion, ne peut, selon nous, relever que d'une intervention anthropique ${ }^{6}$.

Le cas de l'individu déposé dans la fosse 17 est encore plus spectaculaire: l'individu n'est que partiellement représenté par le crâne, les membres supérieurs et les mains, les deux clavicules, une fibula, quelques côtes et vertèbres (fig. 15). La présence de nombreux os en dehors du volume corporel indique une décomposition en espace vide. Les quatre premières vertèbres cervicales, en connexion stricte, ainsi que la connexion préservée de l'atlas et du bloc craniofacial permettent d'envisager, ici aussi, un dépôt primaire et une intervention anthropique sur un corps en cours de décomposition, intervention se traduisant notamment par le prélèvement d'importants segments anatomiques (le bassin et la plus grande partie des membres inférieurs notamment). Le positionnement particulier des clavicules, déposées côte à côte à proximité du crâne, ne laisse que très peu de doutes quant au caractère anthropique de l'intervention.

Le même constat vaut pour la structure 650/2 de Marlenheim (site $n^{\circ} 18$ ) : il s'agit d'une «tombe à étage» ayant livré deux niveaux d'inhumation. Au niveau supérieur figurait un adulte en position conventionnelle (650/1) ; sous cette inhumation, gisait un second individu en position primaire, privé de la totalité de son bassin et de ses membres inférieurs. Sa localisation, dix centimètres à peine sous le corps complet du second inhumé, permet d'exclure l'hypothèse d'un remaniement récent et d'envisager, ici aussi, la possibilité d'une manipulation d'origine anthropique.

6. Aucune trace d'activité d'animaux fouisseurs, parfois suspectés d'être à l'origine de tels déplacements - ce qui peut d'ailleurs être discuté lorsqu'il s'agit d'éléments anatomiques importants - n'a été mise en évidence ici. 

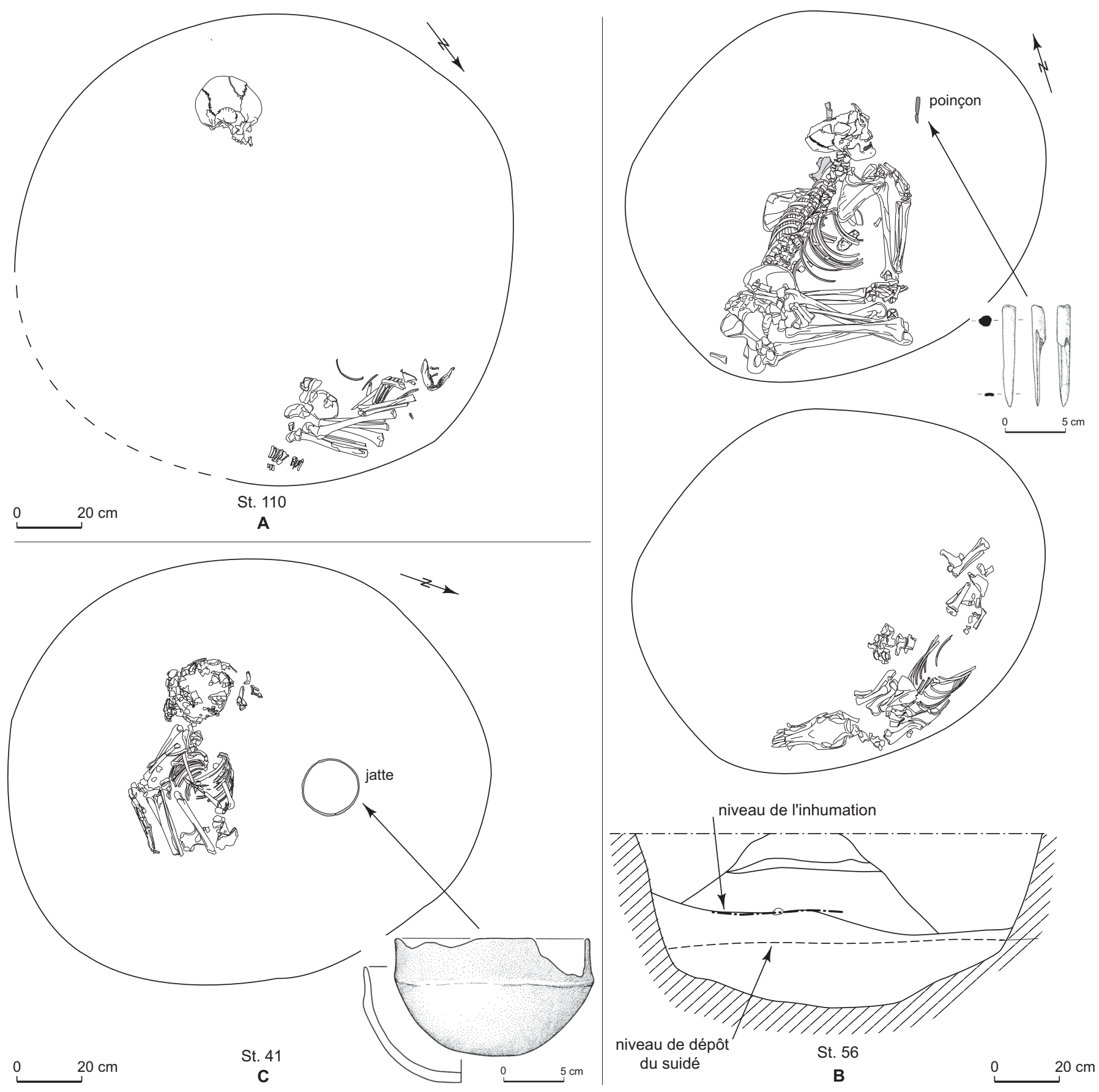

Fig. 14 - L’Aérodrome à Colmar: A, inhumation 110; B, l'inhumation et le dépôt de porc de la structure 56; C, inhumation 41 (DAO: Ch. Leyenberg, Inrap; dessins du mobilier: Ph. Lefranc, Inrap).

La mise en évidence de ce type de manipulation permet de répondre, au moins en partie, à la question soulevée par la présence des segments anatomiques en position secondaire dans des fosses de plan circulaire.
Il est plus que probable qu'au sein du corpus régional, d'autres inhumations en dépôt primaire dans des fosses circulaires aient fait l'objet d'interventions postdécompositionnelles discrètes, mais le caractère ancien de la plupart 


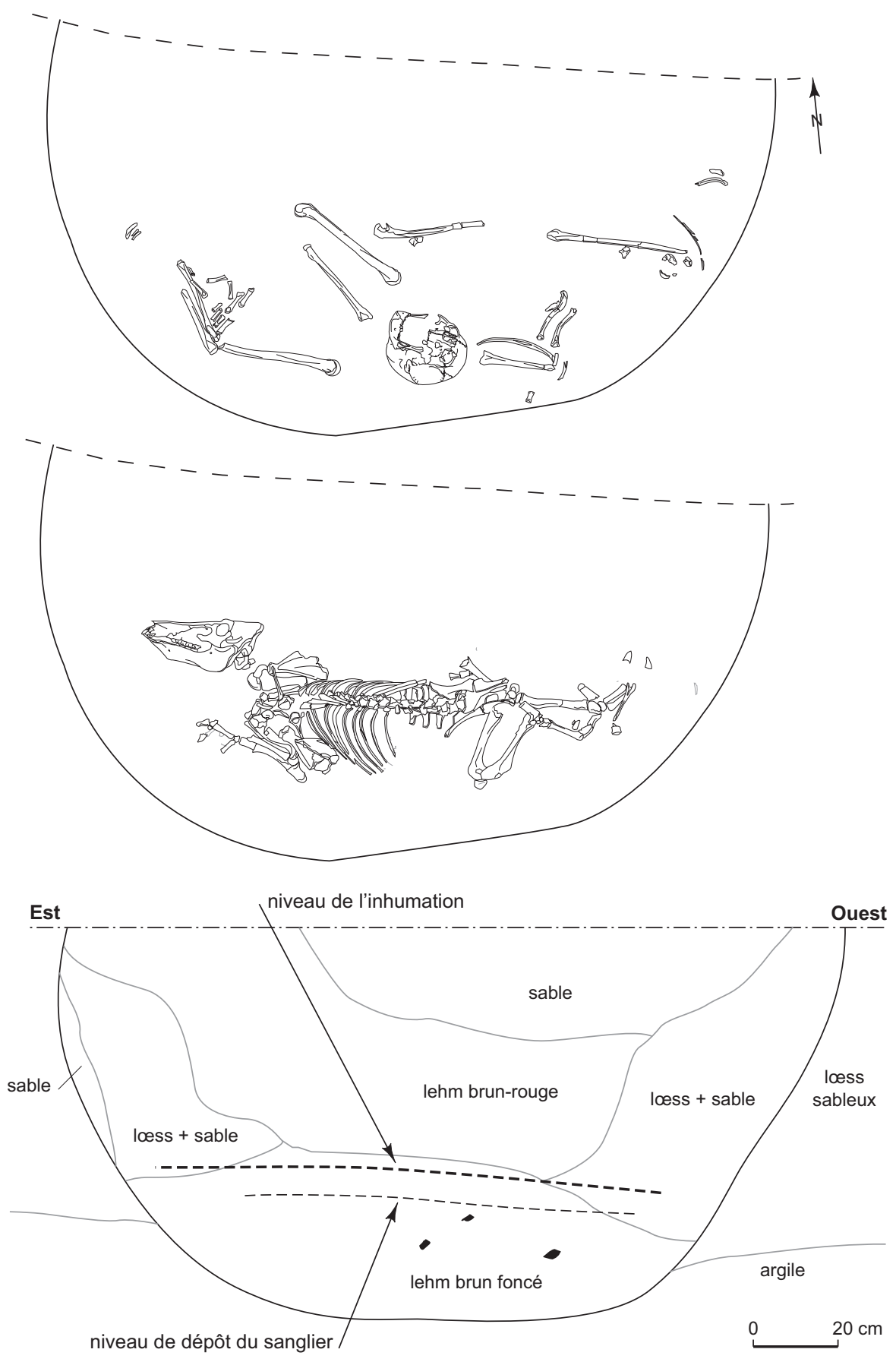

Fig. 15 - L’Aérodrome à Colmar, linhumation et le dépôt de sanglier de la structure 17 (DAO: Ch. Leyenberg, Inrap).

des découvertes ne facilite pas l'identification de tels gestes. Les manipulations de crânes sont par nature moins difficiles à identifier. À Didenheim/Morschwiller-le-Bas, cas déjà mentionné, le bloc craniofacial de l'enfant reposant en position repliée sur le fond de la fosse 8/zone 2, a clairement été prélevé après décomposition des chairs comme l'indique un petit fragment crânien demeuré en place (Denaire, 2007). C'est probablement également le cas là où sont signalés des squelettes acéphales, à Kiechlinsberg (Nickel, 1998) et à Aue à Bruchsal (fosse 5).

D'autres exemples régionaux montrent une position aberrante du crâne, dissocié du reste du squelette et 
repositionné à proximité: cette configuration s'observe notamment à Forlen à Geispolsheim (site n ${ }^{\circ}$ ) et à Entzheim (site $\left.n^{0} 4\right)^{7}$. À Entzheim, la déconnexion du crâne, de l'atlas et de l'axis a amené F. Lambach à évoquer l'existence d'une structure périssable qui aurait surélevé la tête lors de l'inhumation ${ }^{8}$; on pourrait également proposer une interprétation faisant intervenir une manipulation postdécompositionnelle mais les observations taphonomiques disponibles sont insuffisantes.

L'individu mis au jour à Forlen à Geispolsheim offre un exemple très convaincant - quoique difficile à discuter dans le détail - de manipulation: le crâne de cette femme adulte repose, en connexion avec la mandibule, contre le bord externe des premières côtes droites. D'après l'étude taphonomique réalisée par E. Boës, seule une intervention anthropique sur le corps frais (décollation) ou en cours de décomposition, peut expliquer sa position particulière (Billoin et al., 2005).

L'intérêt porté aux crânes dans la gestuelle mortuaire du Néolithique récent apparaît aussi dans les dépôts observés dans quelques fosses-silos, notamment sur les sites d'Am Schluesselberg à Holtzheim (site $\mathrm{n}^{\mathrm{o}} 12$ ), de Hofstatt à Marlenheim (site $\mathrm{n}^{\circ} 18$ ), de Trummelmatten à Merxheim ( site $n^{\circ} 36$ ) et de l'Aérodrome à Colmar (site no 30). Sur ces deux derniers sites, les crânes étaient accompagnés d'éléments de parure: respectivement un collier de perles et un pendentif sur défense de suidé.

Ces exemples trouvent d'intéressants parallèles à Michelsberg à Untergrombach où, s'il faut en croire les données publiées par Bonnet à la fin du XIX $^{\mathrm{e}}$ s., les deux crânes déposés dans la fosse 25, entourés de couronnes de pierres, ont véritablement été mis en scène; rappelons également le dépôt observé dans la fosse 50 du même site (Bonnet, 1899).

Les fossés Michelsberg livrent régulièrement, parmi des restes humains fragmentaires, des crânes bénéficiant d'un traitement spécifique: les exemples les plus spectaculaires sont ceux de Hetzenberg à Neckarsulm-Obereisesheim et d'Altenberg à Heidelsheim (Kreis Bruchsal), où les crânes apparaissent regroupés, en nombre variable, au niveau des têtes des segments de fossés. Ils sont fréquemment associés à

7. La déconnexion du crâne et de la mandibule de l'adulte de la fosse 1 de Didenheim/Morschwiller-le-Bas ne semble pas d'origine humaine, mais a été provoquée, d'après les fouilleurs, par le passage d'un fouisseur (Denaire, 2007).

8. L'hypothèse d'un support en matière périssable a également été évoquée pour la tombe Michelsberg ancien de Matzenheim (Toullec, 2008). des vases presque entiers, caractère se retrouvant également plus à l'est, sur le site d'Altheim (Nickel, 1998).

\section{LE REGRUTEMENT}

L'étude du recrutement au sein d'un cimetière ou d'une nécropole permet classiquement de mettre en évidence d'éventuelles anomalies dans le profil de mortalité de la population inhumée et donc de se rendre compte si elle est représentative d'une population naturelle. Autrement dit, la population inhumée a-t-elle subi une sélection, motivée par l'âge ou le sexe des défunts?

Afin de mener à bien ce type d'étude, il est nécessaire que le corpus disponible soit conséquent et que les méthodes d'estimation de l'âge au décès et de détermination du sexe soient fiables.

Compte tenu de la durée considérée (600 ans), de la dispersion géographique des découvertes et de la part importante des squelettes n'ayant pas bénéficié d'études anthropologiques, il paraît aventureux de se lancer ici dans une analyse détaillée du recrutement.

En effet, les seuls individus pour lesquels nous connaissons les méthodes employées pour mener à bien l'étude biologique proviennent de sites fouillés récemment et ne constituent qu'une minorité du corpus (28 individus adultes et 10 individus immatures) :

- Forlen à Geispolsheim: 1 adulte (site no 9 ) ;

- Gougenheim, diagnostic LGV 2008: 2 adultes (site no 10);

- Altmatt, les Abattoirs et Am Schluesselberg à Holtzheim: 4 adultes (site $\left.\mathrm{n}^{\circ} 12\right)$;

- Complexe sportif d'Ingenheim, diagnostic LGV 2008: 1 adulte (site $\left.\mathrm{n}^{\circ} 14\right)$;

- Contournement routier de Marlenheim: 1 adulte (site $\left.\mathrm{n}^{\mathrm{o}} 17\right)$;

- Hofstatt à Marlenheim: 3 adultes et 2 immatures (site $\left.\mathrm{n}^{\circ} 18\right)$;

- RMS/rue Ampère à Reichstett: 3 adultes et 1 immature (site $\left.n^{\circ} 23\right)$;

- Leimen à Rosheim: 4 adultes et 3 immatures (site $\left.n^{\circ} 24\right)$;

- Aérodrome à Colmar: 9 adultes et 10 immatures (site $\left.\mathrm{n}^{\circ} 30\right)$.

Pour ces individus, les méthodes utilisées pour déterminer le sexe sont relatives à l'os coxal (Bruzek, 2002 ou Murail et al., 2005), qui est le seul os du squelette humain permettant une diagnose fiable du fait de son adaptation morphologique, pour les femmes, à la parturition. L'âge des immatures est estimé par l'intermédiaire de la minéralisation dentaire (Moorrees et al., 1963a et b) ou, à défaut 
de restes dentaires, de la croissance osseuse (Maresh, 1970 cité par Scheuer, Black, 2000) et celui des adultes par l'intermédiaire de la maturation osseuse (Owings-Webb, Suchey, 1985).

Il est aisé de se rendre compte que le nombre d'individus ayant bénéficié de telles méthodes est trop faible pour mettre en place une étude fiable et pertinente du recrutement.

Le corpus disponible en Alsace est cependant prometteur. D’après les données issues des fouilles anciennes, 93 individus adultes, dont 26 hommes et 21 femmes, et 44 individus immatures ont été mis au jour. Il s'avère aujourd'hui indispensable d'effectuer une révision du matériel ostéologique en utilisant les méthodes reconnues actuellement comme étant les plus fiables et les mieux adaptées afin de pouvoir procéder à une étude approfondie du recrutement pour cette période, dans cette région.

La fouille récente du site de Gougenheim, situé sur le tracé de la ligne LGV Est et pour laquelle l'étude anthropologique est en cours, va également permettre d'enrichir les données actuelles en doublant le corpus actuellement disponible (site $\mathrm{n}^{\mathrm{o}} 10$ ).

La population inhumée pourra alors être étudiée, tout d'abord dans sa globalité (proportion d'immatures par rapport aux adultes; taux de masculinité), puis en fonction des modes d'inhumation au sein des différentes cultures ou aires culturelles identifiées.

\section{LES TRACES DE VIOLENGE}

La reconnaissance de la pratique de l'accompagnement au Néolithique récent pose implicitement la question de la mise à mort des accompagnants. Bien entendu, cette dernière ne laisse pas nécessairement de traces sur les os. Il est toutefois légitime de poser cette question et de rechercher sur les cadavres les indices de violence. Malheureusement, peu de squelettes, même parmi les découvertes récentes, ont bénéficié d'un tel examen. Il est donc impossible d'estimer la fréquence de ces traces. Cette lacune est d'autant plus regrettable que l'étude entreprise sur les squelettes de Leimen à Rosheim montre qu'une telle approche n'est pas vaine (Lefranc et al., 2007). À notre connaissance, en Alsace, six individus dont trois adultes et trois enfants, présentent des traces de coups violents potentiellement mortels, tous portés sur le crâne (Boës, 2007) :

- Lerchenberg à Didenheim, F13: 1 enfant avec un enfoncement du crâne (site ${ }^{\circ} 31$ );

- Hausbergen à Mundolsheim, MAS 28014: 1 adulte avec enfoncement de l'arrière-crâne (site $\left.n^{\circ} 22\right)$;
- Violette à Riedisheim: 1 adulte avec un enfoncement du crâne (site $\left.n^{\circ} 38\right)$;

- Leimen à Rosheim, st. 107: 1 enfant avec un enfoncement de l'arrière-crâne (site $\left.n^{\circ} 24\right)$;

- Leimen à Rosheim, st. 171: 1 enfant avec un enfoncement du crâne;

- Lotissement Sainte-Odile à Rosheim : 1 femme adulte avec un enfoncement du crâne (site $n^{\circ} 25$ ).

\section{LES DÉPÔTS DE MOBILIER ASSOCIÉS AUX RESTES HUMAINS}

On a, depuis les premières découvertes d'inhumations en fosses rondes, beaucoup discuté du rituel funéraire, l'une des principales questions étant d'établir si le mobilier recueilli dans les différents niveaux des creusements était à mettre en rapport avec les funérailles ou s'il s'agissait d'un mobilier d'origine détritique. La première hypothèse a été défendue dès le début des années 1970 par R. Schweitzer pour qui la présence de fonds rubéfiés et de couches de charbons trahissait un rituel funéraire à Riedisheim (site $n^{\circ} 38$ ) et à Didenheim (site $n^{\circ} 31$ ). La même thèse a été soutenue par J. Lichardus, avec des arguments proches de ceux mis en avant par R. Schweitzer (Lichardus, 1986).

Soutenant une autre hypothèse, C. Jeunesse a souligné, d'une part, que le mobilier figurant dans ces silos est le plus souvent pauvre et d'une composition tout à fait comparable à celui recueilli dans les fosses d'habitat et, d'autre part, que la présence d'un fond rubéfié ou de couches de cendres ne constituait aucunement un argument déterminant, ces caractères relevant davantage d'une technique d'assainissement et de consolidation des silos céréaliers (Jeunesse, 1982). Les découvertes de Bruechel à Geispolsheim ont, quelques années plus tard, définitivement démontré que ces aménagements n’ont rien de spécifiquement funéraire (Jeunesse, Sainty, 1986). La fouille récente du site de l'Aérodrome à Colmar (site $\left.n^{\circ} 30\right)$ permet d'apporter un nouvel élément déterminant à cette discussion: ce site riche en inhumations primaires et en dépôts secondaires, constitué de plus de 70 fosses de type silo, n'a livré aucun élément détritique. Ces creusements, localisés à l'écart des zones d'activités, n’ont jamais été réutilisés à d'autres fins que «funéraires» et ne présentent aucun des caractères autrefois mis en avant par Lichardus ou Schweitzer.

Nous considérons comme dépôts avérés les objets complets ou subcomplets localisés au même niveau que le squelette. Ils sont généralement constitués d'un ou deux objets, essentiellement des récipients en céramique, plus 
rarement des objets en os ou en bois de cerf ou du matériel de mouture. Aujourd'hui, les dépôts funéraires attestés concernent environ $10 \%$ des inhumations en fosses rondes du sud de la plaine du Rhin supérieur.

La catégorie la plus fréquente est la céramique que l'on rencontre à cinq reprises: à Didenheim/Morschwillerle-Bas (site no 33; fig. 11), sur le site RMS/rue Ampère à Reichstett (site $n^{\circ}$ 23), à l'Aérodrome à Colmar (fig. 14, C; site $n^{\circ} 30$ ), à Leimen à Rosheim (site $\left.n^{\circ} 24\right)$, et à Hofstatt à Marlenheim (site $n^{\circ} 18$ ).

Les dépôts sont constitués d'un ou deux récipients de type jatte (3 occurrences) ou gobelet (2 occurrences). On note que la majorité des récipients n'ont pas été déposés entiers mais ont été préalablement brisés. Ce rituel qui marque déjà, à l'échelle régionale, la grande majorité des dépôts funéraires du Néolithique ancien et moyen, est signalé dans la fosse 102 du site RMS/rue Ampère à Reichstett où une jatte complète associée à quelques fragments d'une grande jarre ont été intentionnellement déposés à proximité du corps (site no 23), ainsi qu'à la Rocade ouest de Didenheim/ Morschwiller-le-Bas où seule une moitié de jatte a été dépo-

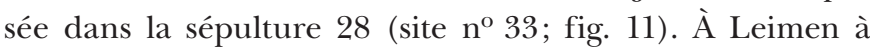
Rosheim, l'individu inhumé dans la fosse 94 était accompagné par un tulipiforme entier et par un grand fragment de Beutelbecher (fig. 7; site $\mathrm{n}^{\mathrm{o}}$ 24). Enfin, le dépôt de Hofstatt à Marlenheim consiste en une moitié de gobelet, elle-même brisée en deux fragments dont l'un a été déposé au contact de l'individu en position fléchie (site $\mathrm{n}^{\mathrm{o}} 18$ ).

En contexte Michelsberg, le dépôt de récipients préalablement brisés se rencontre également sur le site de Grossvillars à Oberderdingen (Landkreis Karlsruhe), où deux grands fragments de bouteille à couronne d'anses ont été placés sur le crâne et sur le bassin d'un individu inhumé dans une fosse-silo (Stauch, Banghard, 2002).

Outre-Rhin, toujours en contexte Michelsberg, les inhumations en fosses rondes ayant livré des céramiques - plus ou moins bien documentées -, sont au nombre de six. À l'exception de la bouteille d'Oberderdingen, les types représentés sont les mêmes qu'en Alsace et les dépôts ne sont constitués que par un unique objet: on note une jatte carénée à Im Tale Links à Bad-Kreuznach, des gobelets tulipiformes déposés chaque fois en un unique exemplaire à Nauberg à Bad-Kreuznach, Stuttgart-Münster et Wolfenweiler-Leuterberg (Nickel, 1998).

Les objets en matières dures animales apparaissent dans quatre sépultures régionales: il s'agit des outils en os observés à Colmar (site no 30 ; fig. 14, B), à la Sablière Lienhardt à Lingolsheim (site $\left.\mathrm{n}^{\circ} 16\right)$ et à l'Aéroparc à
Entzheim (sép. 1266; site $\mathrm{n}^{\circ} 6$ ), ainsi que d'un gobelet en bois de cerf importé de Suisse occidentale et déposé auprès de l'inhumation principale de la st. 28 de Didenheim/ Morschwiller-le-Bas (site $\mathrm{n}^{\circ} 33$; fig. 11). D'autres gobelets du Cortaillod ont été découverts dans une minière de Kachelfluh-Höhle à Kleinkems (site $n^{\circ} 45$ ), dans une petite niche accueillant les restes partiels de plusieurs individus (Nickel, 1998).

Des parures apparaissent en position fonctionnelle, à Altmatt à Holtzheim (un collier composé de perles cylindriques en calcaire, d'un galet plat perforé et de coquillages; site $n^{\circ} 12$ ) et à Didenheim/Morschwiller-leBas (zone 2/st. 8: un galet perforé et deux craches de cerf; site $\left.n^{\circ} 33\right)$. On rencontre également des parures déposées auprès de crânes isolés, dans la fosse 57 de Trummelmatten à Merxheim (site $n^{\circ}$ 36) et à l'Aérodrome à Colmar (une défense de suidé perforée). Le collier de Merxheim est composé de onze perles dont quatre en forme de hache qui évoquent les exemplaires Cortaillod ancien de Suisse occidentale.

Enfin, trois structures ont livré du matériel de mouture, au Lotissement Sainte-Odile à Rosheim (site no 25), à Leimen à Rosheim, st. 104 (site $n^{\circ}$ 24) et à Marlenheim (site $\mathrm{n}^{\mathrm{o}}$ 18). À Rosheim, la meule a été déposée au même niveau et au contact de l'individu inhumé. Ce n'est le cas ni à Rosheim ni à Marlenheim où le matériel de mouture repose sur le fond du creusement, à quelques centimètres au-dessous des corps; cependant les similitudes existant entre ces deux ensembles nous amènent à nous demander s'il ne s'agit pas là de dépôts funéraires plutôt que de rejets domestiques.

Pour conclure cet inventaire, nous soulignerons que les dépôts funéraires sont, sans exception aucune, destinés à des individus en position conventionnelle. Dans le cas des sépultures plurielles ayant livré des dépôts funéraires (Didenheim et Marlenheim), ces derniers sont clairement associés aux seuls individus en position contractée.

\section{DES DÉPÔTS NON FUNÉRAIRES ?}

Les corps en position non conventionnelle se rencontrent en majorité dans le cadre des inhumations multiples, nous l'avons vu, mais il existe également des exemples de corps isolés. Pour s'en tenir au Rhin supérieur, citons les exemples de la fosse Knobel II/3 à Munzingen (site $\mathrm{n}^{\circ} 47$ ), de la fosse 2 de Lerchenberg à Didenheim (site $n^{\circ} 31$ ), de la F.152 RMS/rue Ampère à Reichstett (site $n^{\circ} 23$ ) de Gougenheim/st. 52 (site $n^{\circ} 10$ ), Ingenheim (site $n^{\circ} 14$ ) et de 


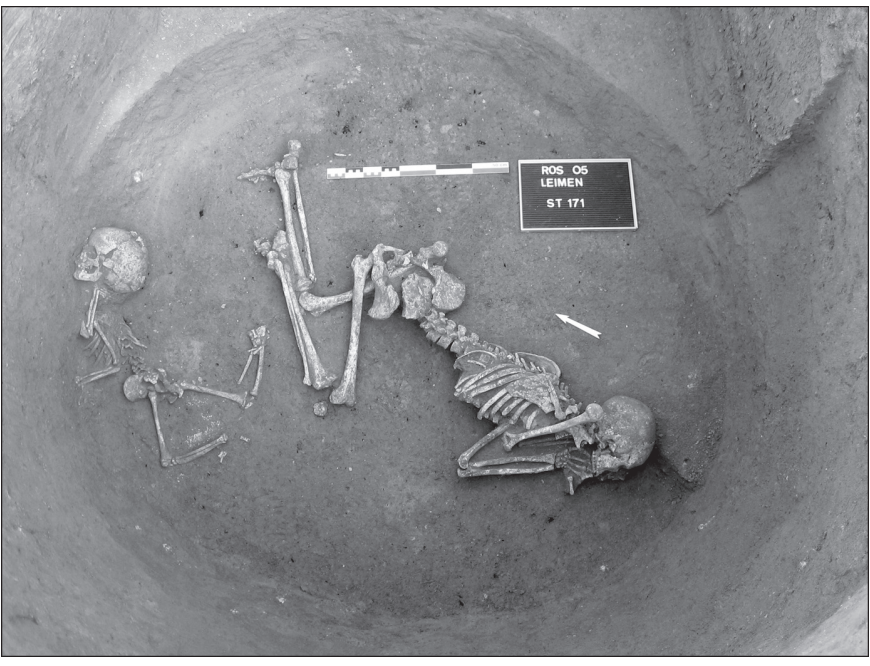

Fig. 16 - Leimen à Rosheim, vue des deux individus en position non conventionnelle de la structure 71 (cliché: Ph. Lefranc, Inrap).

l'Aérodrome à Colmar (site no 30). Nous connaissons également un ensemble, à Leimen à Rosheim/st. 171 (fig. 16; site $\mathrm{n}^{\circ} 24$ ), où figurent deux corps en position non conventionnelle: une femme adulte en procubitus et un enfant portant de très nettes traces de fracturation crânienne, reposant sur un même niveau et, selon toute probabilité, déposés simultanément (Lefranc, Boës, 2006; Lefranc et al., 2007).

L'interprétation de ces dépôts d'individus affectant la même position désordonnée que les «accompagnants" des inhumations multiples est délicate. On peut poser l'hypothèse de fosses "satellites de la sépulture principale " (Boulestin, 2008), en rappelant que, sur la plupart des sites explorés sur de grandes surfaces, figurent également des inhumations d'individus en position conventionnelle; la proposition paraît recevable mais elle est difficilement démontrable.

Seconde hypothèse, il pourrait s'agir de sépultures de relégation dans le sens défini par A. Villes pour les inhumations en silos de La Tène ancienne (Villes, 1986). L'idée est celle de l'existence de plusieurs degrés du rituel funéraire, les inhumations en silos, celles d'individus n'ayant pas accès à la nécropole, occupant l'échelon le plus bas. Pour le Néolithique récent, on peut retenir de cette proposition l'hypothèse d'une gradation du rituel pouvant se traduire par des gestes très particuliers - ou par une absence de gestes autres que le dépôt en silo - réservés à certaines catégories de la population auxquelles la position «conventionnelle» est refusée. Si l'on envisage un instant cette possibilité, il

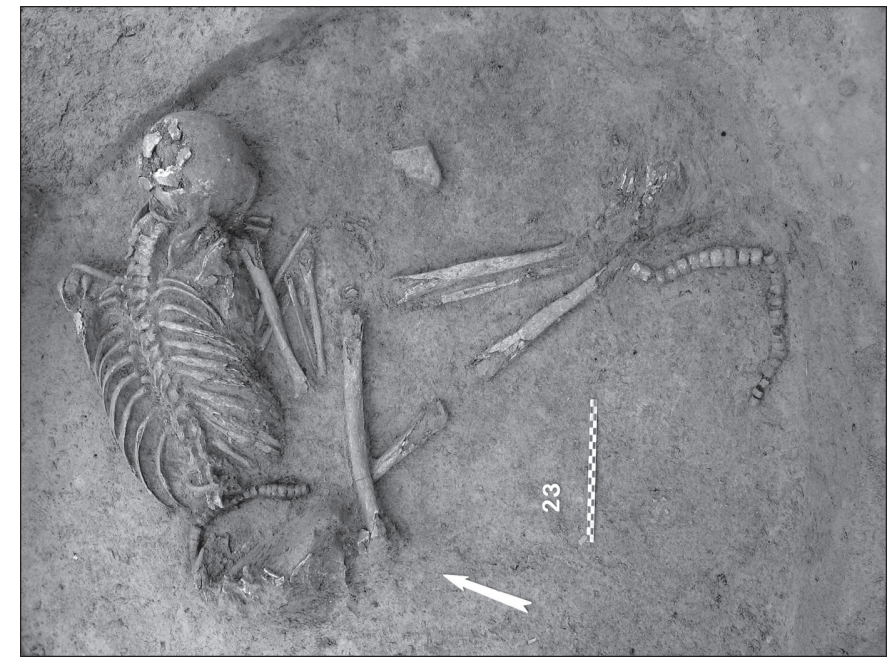

Fig. 17 - L'Aérodrome à Colmar, vue de l'individu en position non conventionnelle de la structure 23 et des deux «colliers» de perles en cuivre in situ (cliché: Ph. Lefranc, Inrap).

faut alors admettre que ces mêmes catégories sont fortement impliquées dans le rituel de l'accompagnement.

On sait que l'hypothèse de la relégation sociale à La Tène est mise en concurrence par certains protohistoriens avec celle des «silos-offrandes», concept forgé récemment et impliquant l'offrande aux dieux d'un individu ou d'un animal à forte valeur symbolique et sociale (Delattre et al., 2000); c'est la troisième hypothèse.

La découverte récente à l'Aérodrome à Colmar d'un individu inhumé en position désordonnée et accompagné de deux objets composés de perles en cuivre (fig. 17; site $\mathrm{n}^{\circ} 30$ ) nous amène à ouvrir le débat pour le Néolithique récent. Le sujet repose en procubitus dans le remplissage d'une fosse circulaire (st. 23A), membre inférieur gauche tendu formant un angle de $90^{\circ}$ avec le tronc, et cuisse droite fléchie sur la droite du tronc. Au même niveau et, dans un cas, en contact strict avec le corps, ont été découverts deux «colliers» totalisant 56 perles en cuivre de type Mondsee pour un poids d'environ $400 \mathrm{~g}$, appartenant à un type bien connu dans le Cortaillod tardif (Sangmeister, Strahm, 1971; Ottaway, Strahm, 1974). L'assimilation de ces objets à des parures ne va pas de soi: certains auteurs les identifient à des lingots, voire à une protomonnaie utilisée dans le cadre de transactions sociales (Ottaway, Strahm, 1975). Quel que soit leur statut, ces deux objets importés doivent être considérés comme rares et investis d'une forte valeur sociale (Pétrequin, Pétrequin, 1993; Klassen et al., 2007).

L'improbable association entre objets socialement valorisés et corps en position non conventionnelle nous incite 
à considérer comme très faible l'hypothèse du dépôt funéraire dans la mesure où, nous l'avons souligné, tous les dépôts d'objets avérés accompagnent des individus en position conventionnelle. L'hypothèse d'un «accompagnant» déposé dans une fosse satellite, qui n'explique pas la présence de ces objets, ne nous paraît pas beaucoup plus satisfaisante et, enfin, il semble que l'on puisse écarter sans trop de discussion l'éventualité d'une sépulture de relégation: si l'absence éventuelle de gestes funéraires pour les individus inhumés en position «inorganisée» peut aller dans le sens de la relégation sociale, rien ne semble justifier, dans cette hypothèse, la présence à leurs côtés d'objets socialement valorisés.

S'agissant d'un unicum, nous ne pouvons malheureusement que spéculer sur le sens de ce dépôt qui nous amène néanmoins à nous interroger sur l'éventuelle existence, au Néolithique récent, de dépôts humains réalisés en dehors du cadre funéraire. Si l'on écarte l'hypothèse funéraire, comme nous avons tendance à le penser, il faut se tourner vers d'autres types de motivations, notamment vers celles pouvant justifier l'enfouissement d'objets socialement valorisés. Ces dépôts qui traversent tout le Néolithique (Lichardus-Itten, 1991 ; Jeunesse, 1998; Klassen et al., 2007) ont été abondamment étudiés et ont fait l'objet d'interprétations diverses: on retiendra ici celle de l'offrande faite à des «puissances surnaturelles» (Pétrequin et al., 2002), et celle qui évoque un aspect secondaire de la cérémonie du potlatch durant laquelle la destruction somptuaire de certains biens intervient dans le cadre de la compétition sociale entre élites (Klassen, 2001). Les deux propositions ne sont d'ailleurs pas tout à fait antagonistes puisque, si l'on se réfère au texte de $\mathrm{M}$. Mauss, ces destructions sont «très souvent sacrificielles» et «bénéficiaires pour les esprits » qui assistent aux cérémonies (Mauss, 1924-1925, éd. 2007).

En l'absence de référent, la plupart des hypothèses formulées plus haut ne peuvent être, en toute objectivité, définitivement écartées (notamment celle de l'accompagnement), mais nous remarquerons néanmoins, tout en gardant la distance nécessaire, que dans une société à haut degré d'intégration et qui pratique de surcroît l'accompagnement funéraire, comme cela semble être le cas pour nos sociétés du Néolithique récent/Chalcolithique ancien, l'hypothèse de la mise à mort d'individus aux cours de cérémonies non funéraires n'a en théorie rien d'irrecevable: en témoignent les sociétés de la côte nord-ouest du Canada où l'accompagnement est attesté et où la mise à mort ostentatoire d'esclaves, considérés comme éléments de richesse, est une des composantes des potlatch de destruction (Testart, 2001).
Le problème se pose dans les mêmes termes pour les dépôts d'animaux retrouvés sur les sites régionaux dont nous ne savons s'il faut, le cas échéant, les relier à des inhumations proches ou les considérer comme des dépôts en tant que phénomène à part entière et sans rapport nécessaire avec les gestes funéraires.

\section{LES DÉPÔTS D’ANIMAUX}

\section{LE CORPUS RÉGIONAL}

L'un des traits marquants relevés sur plusieurs des sites régionaux concernés par la pratique des inhumations en fosses est représenté par la découverte de squelettes d'animaux complets ou de parties de squelettes. Les données les plus récentes et les mieux documentées à ce sujet concernent les sites de Leimen à Rosheim et de l'Aérodrome à Colmar (sites n ${ }^{\text {os }} 24$ et 30).

Dans le premier cas, à Rosheim, un squelette d'un faon de cerf a été disposé à mi-hauteur du comblement du silo 74, contre le bord ouest de la fosse (fig. 18). L'état d'éruption dentaire (d4 en tout début d'usure et M1 inférieure en cours de sortie) permet d'estimer qu'il s'agit d'un jeune de 4 à 5 mois, soit un animal mort à la fin de l'été. Il s'agit d'un squelette complet dont la totalité des os a pu être collectée, y compris les plus discrets comme les sésamoïdes ainsi que l'ensemble des parties des extrémités et les plus fragiles comme les sternèbres et les cartilages costaux. La position de l'animal, enroulé sur lui-même, les quatre membres réunis sous lui, reflète un soin particulier et laisse penser qu'il s'agit d'un dépôt intentionnel plutôt que d'un cadavre évacué dans une fosse en cours de comblement. Dans la partie inférieure du remplissage, à quelques centimètres en dessous du dépôt de ce faon, a été mise au jour une hache perforée en bois de cerf, ainsi qu'un fragment de plat à cuire et un bord de vase typique du Munzingen. Le silo 87, localisé au nord du site, a livré les restes du squelette complet d'un chien assez âgé d'après l'état d'usure des dents. Les ossements ne présentent pas plus de traces d'exploitation que ceux du squelette du faon. L'animal reposait, plaqué contre la paroi, à $10 \mathrm{~cm}$ au-dessus du fond du silo. La position de l'animal, les membres et le cou tendus évoquent cependant une manipulation un peu moins précautionneuse que dans le cas du squelette de faon, comme si l'animal avait été saisi par ses pattes pour être disposé dans la fosse sur le dos. Les membres inférieurs étaient posés sur un fragment de meule en grès. Dix centimètres au-dessus du squelette de chien, à proximité de la paroi nord, étaient 


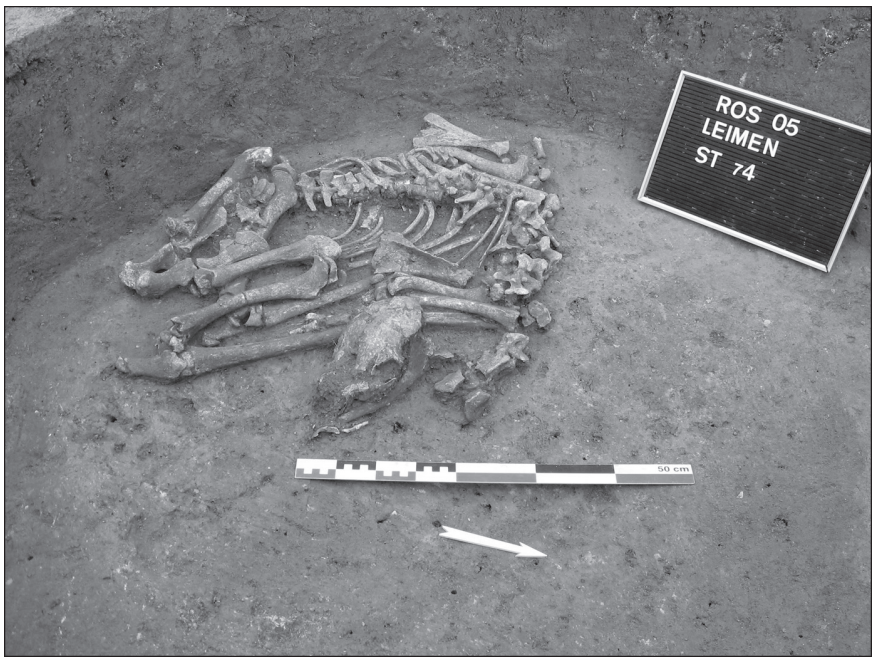

Fig. 18 - Leimen à Rosheim, vue du dépôt de faon de la structure 4 (cliché: Ph. Lefranc, Inrap).

disposés les restes, en connexion, des extrémités de deux pattes postérieures d'un jeune porc (fig. 19). Un peu plus haut et dans le même secteur figurait la partie supérieure d'un crâne de porc reposant sur sa face supérieure.

Sur le site de l'Aérodrome à Colmar, plusieurs fosses circulaires intègrent, à des niveaux de remplissage variables, des ensembles d'ossements d'animaux:

- le squelette d'un jeune cerf, disposé dans le comblement inférieur du silo 33 (fig. 20). Il s'agit d'une dépouille complète dont la plupart des éléments sont en connexion anatomique et se présentent en position primaire à l'exception du crâne complètement disloqué, dont les différentes parties (mandibules, occipital...) sont isolées et dispersées sur toute la surface du niveau de dépôt. Le squelette appendiculaire est complet et en connexion anatomique à l'exception de l'extrémité du membre antérieur gauche (à partir du métacarpe) et des éléments supérieurs (humérus, radius) isolés et éparpillés. L'âge de l'animal peut être estimé à 4-5 mois d'après l'état d'éruption et d'usure de la dentition (d4 en début d'usure, M1 en cours de sortie);

- des restes du rachis et d'éléments crâniens d'un autre jeune cerf dans le silo 48. Il s'agit principalement des vertèbres thoraciques et de côtes en connexion, de deux vertèbres cervicales isolées, des deux mandibules et du crâne très fragmenté et réduit à quelques-unes de ses parties (occipital, zygomatiques...) L'âge estimé de l'animal est très proche de celui de la fosse 33 (d4 en tout début d'usure et M1 en cours de sortie);

- les restes du squelette d'un jeune sanglier dans la fosse 17 (fig. 21) disposé sur le côté droit, les membres

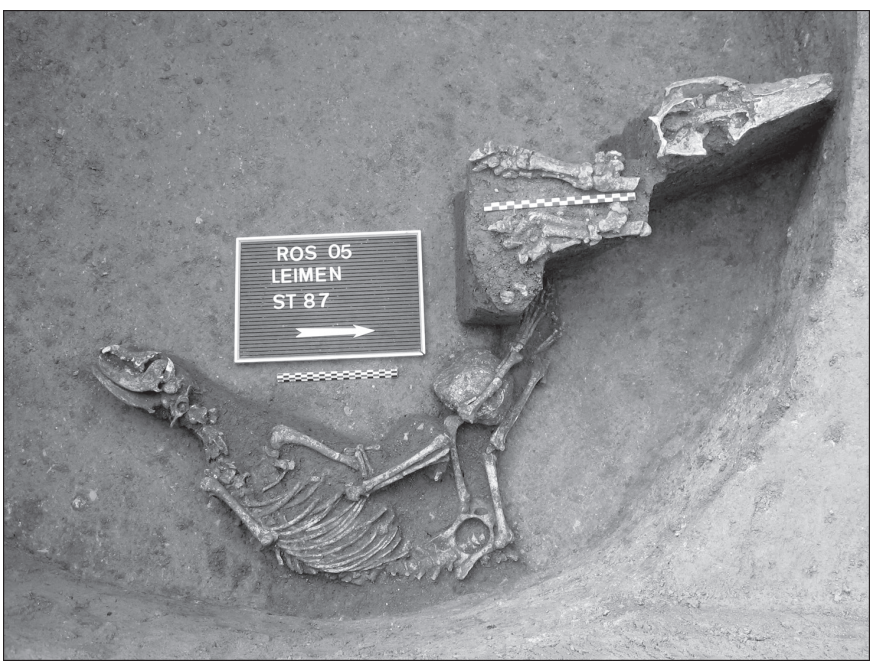

Fig. 19 - Leimen à Rosheim, vue des dépôts de la structure 87: chien, pattes postérieures et crâne de porc (cliché: Ph. Lefranc, Inrap).

antérieurs repliés et les membres postérieurs en extension. Il s'agit d'un mâle d'environ 10-12 mois (M2 en cours de sortie), dont la plupart des parties du squelette sont attestées, à l'exception des vertèbres cervicales (à partir de l'axis), et se présentent en connexion anatomique;

- le squelette incomplet d'un porc domestique, de sexe mâle, dont l'âge peut être estimé à 14-16 mois (M2 en début d'usure, M3 non sortie) en position allongée contre la paroi de la fosse 56. La plupart des éléments du squelette sont attestés, mais la préservation des connexions articulaires se limite au crâne, aux membres antérieurs et à une partie du rachis (fig. 14, B);

- des vestiges très résiduels du squelette d'un suidé de quelques semaines dont seuls les éléments les plus conséquents (principaux os longs, mandibule...) ont été préservés.

L'hypothèse de l'évacuation de dépouilles d'animaux écartés de l'alimentation pour des raisons sanitaires (épizooties, mortalité naturelle) ne peut être purement et simplement éliminée pour expliquer la présence de carcasses complètes d'animaux le plus souvent jeunes. La présence d'animaux sauvages, dont les dépouilles ont été transportées sur le site, incite cependant à explorer d'autres scénarios et à intégrer différentes caractéristiques particulières dont la mise en évidence repose en grande partie sur des méthodes de fouille et des observations de terrain précises.

Ces vestiges forment des ensembles bien individualisés, qui se présentent groupés au sein d'un même niveau, séparés d'autres concentrations de restes lorsque le comblement est formé de dépôts successifs. Cette configuration peut être invoquée dans le cas de la fosse 56 du site de l'Aérodrome 


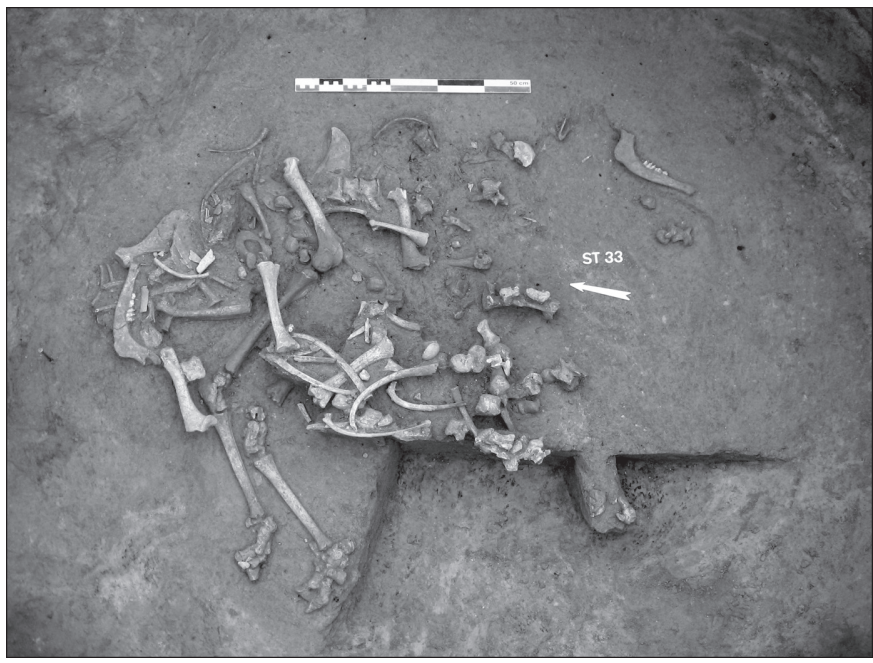

Fig. 20 - L’Aérodrome à Colmar, vue du faon remanié de la structure 33 (cliché: Ph. Lefranc, Inrap).

à Colmar, où une faible épaisseur de sédiment s'intercale entre la carcasse du porc et le squelette humain déposé au-dessus (fig. 14, B). Les espèces concernées restituent une sélection qui ne se superpose pas exactement à celle sur laquelle repose l'alimentation carnée dans laquelle le porc tient une place importante mais qui n'intègre que très marginalement les produits de la chasse du cerf et du sanglier et, de façon encore plus anecdotique, le chien. La disposition du faon de Leimen à Rosheim (fig. 18), de même que celle du sanglier de Colmar (fig. 21), évoque un dépôt soigneux dont l'intentionnalité ne fait guère de doute. La préservation des connexions anatomiques suggère qu'il s'agit de restes en position primaire dont la décomposition a eu lieu sur place et, selon toute vraisemblance, en espace colmaté. Cette caractéristique est loin d'être systématique et ne se vérifie pour aucun des autres squelettes évoqués ci-dessus. Les restes du squelette de faon le plus complet de Colmar (fig. 20) apparaissent partiellement éparpillés. Les mandibules sont séparées du crâne et dispersées, le crâne est déstructuré et les diverses parties (occipital, zygomatiques, prémaxillaires) gisent en position très désordonnée. L'inventaire détaillé des parties anatomiques révèle l'absence de l'extrémité du membre antérieur droit. De même les restes du rachis du faon de la fosse 32 étaient partiellement disloqués (mandibules éloignées de leur position anatomique, déconnexion du crâne) et le rachis apparaît privé des vertèbres cervicales. Le squelette de porc de la fosse 56 est partiellement disloqué; la plupart des vertèbres lombaires sont séparées de la colonne vertébrale et semblent avoir été redisposées à proximité; les principaux os

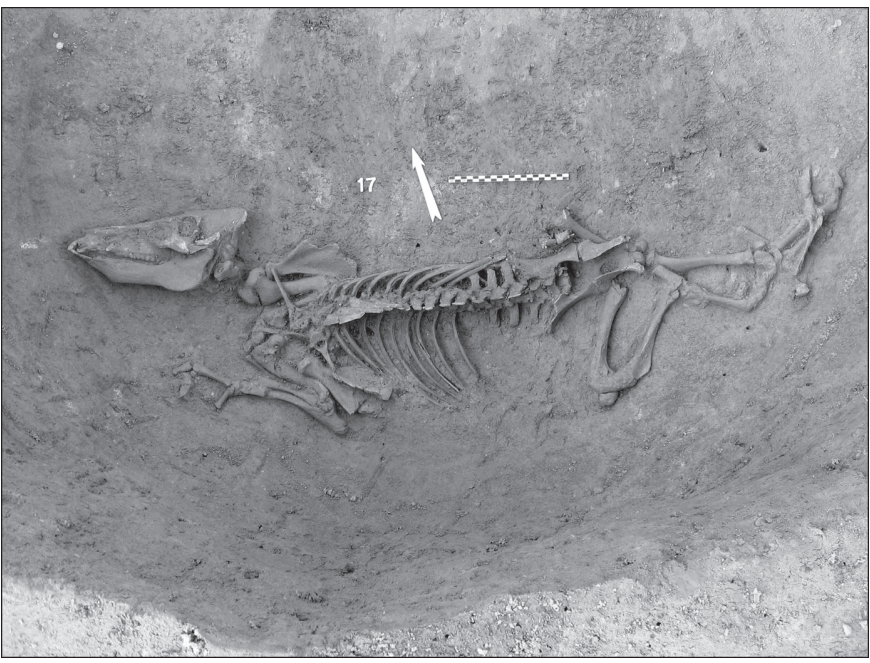

Fig. 21 - L'Aérodrome à Colmar, vue du sanglier de la structure 17 (cliché: Ph. Lefranc, Inrap).

des membres postérieurs sont dissociés et reposent groupés près du squelette dans une position qui évoque une manipulation de type «rangement». L'inventaire anatomique détaillé fait aussi apparaître des lacunes qui concernent principalement des éléments des extrémités (phalanges, carpes, tarses, patella gauche...) qu'il est difficile d'imputer aux techniques de fouille. Ces incohérences dans la répartition des éléments squelettiques et les lacunes dans l'inventaire des parties ne peuvent s'expliquer par les effets de la taphonomie ni par des perturbations occasionnées par des animaux fouisseurs. Elles laissent au contraire entrevoir la possibilité d'interventions postérieures à la décomposition et de manipulations qui se déroulent dans la fosse. Ces remaniements impliquent que les carcasses animales aient pu, dans certains cas, rester accessibles et laissent entrevoir un fonctionnement d'une complexité jusque-là insoupçonnée qui conforte l'idée qu'il s'agit de véritables dépôts dont le fonctionnement présente plus d'une convergence avec celui des dépôts humains.

\section{LE CORPUS DE COMPARAISON}

Ces dépôts de squelettes ou de parties de squelettes d'animaux dans des fosses circulaires peuvent être rapprochés de ceux connus dans un contexte régional plus large. Les suidés et les chiens semblent les plus fréquemment impliqués, alors que d'autres animaux, comme les caprinés, qui n'ont été signalés qu'à Beauséjour à Riedisheim (Schweitzer, Fulleringer, 1973) où des espèces sauvages semblent plus épisodiquement concernés. Ces dépôts impli- 
quent des dépouilles complètes comme à Bruechel à Geispolsheim (Poulain, 1987) où les squelettes de deux jeunes suidés datés du Munzingen occupent les niveaux supérieurs de la fosse 6, à l'Abattoir à Holtzheim avec deux individus (st. 13 et st. 109), à Hofstatt à Marlenheim (structure 166) (Châtelet, 2009) où les restes d'un très jeune porcelet sont directement associés à un dépôt de plusieurs vases ou encore à Forlen à Geispolsheim où les restes d'un individu supplémentaire ont été mis au jour (Lefranc et al., à paraître) et enfin, le suidé découvert en RMS/rue Ampère à Reichstett au-dessus d'une sépulture humaine attribuée au Néolithique récent (site $\mathrm{n}^{\mathrm{o}} 23$ ). L'observation de marques de découpe et de cuisson sur l'un des animaux de Holtzheim et sur les suidés de Rosheim fait défaut ailleurs. La pratique du dépôt de carcasses complètes concerne aussi le chien comme à la Sablière Maetz à Rosheim (Thévenin et al., 1978; Poulain, 1977), où deux individus sont signalés (fosse 2), à Altmatt et aux Abattoirs à Holtzheim (Lefranc, 2001), où deux individus supplémentaires (respectivement st. 22 et st. 10) complètent la série.

Ces comparaisons peuvent être élargies à une échelle géographique qui englobe l'aire d'extension des cultures du Mittelneolithikum et des cultures postérieures du Néolithique récent, soit une zone qui s'étend entre l'Allemagne du sud et l'Autriche occidentale. Sur le site Baalberg de Weissenfels, qui présente la configuration la plus imposante, plusieurs dépôts ont été découverts impliquant pas moins de 24 bovins et une vingtaine de chiens (fosse 27), complétés par 6 crânes de bovins, un crâne de chien et un squelette de chien complet (Preuss, 1966). Sur le site de Ludwigsburg attribué à la culture de Schwieberdingen est attesté un dépôt constitué de 3 squelettes de porcelets, 2 squelettes de jeunes chèvres d'environ 9-10 mois et du squelette d'un chiot sans le crâne (Nobis, 1977). D'autres parallèles peuvent être convoqués dans la culture de Münchshöfen, qui concernent aussi des porcs comme à Burgweinting (Dallmeier, Froschauer, 1995) et à Mamming (Kreiner, Van den Driesch, 1992). Sur ce dernier site, un squelette de chevreuil est également attesté.

Sur bon nombre de ces sites, les dépôts d'ossements animaux apparaissent au voisinage de restes humains, au sein d'une même structure ou dans une fosse distincte mais située à proximité immédiate. Les configurations les plus remarquables sont celles qui réunissent au sein d'une même structure des dépôts de carcasses animales et des squelettes humains comme à Colmar (fosses 17 et 56), à Reichstett, probablement aussi à Riedisheim, sur le site Baalberg de Weissenfels. Dans tous ces cas, les ossements animaux et les vestiges humains forment des ensembles séparés qui résultent d'épisodes successifs, de sorte qu'un lien direct entre les deux types de dépôts ne peut être formellement établi - ce qui ne permet pas de leur conférer une signification funéraire. Si l'on considère cependant que ces dépôts marquent des ensembles de structures plus vastes qui s'organisent autour de sépultures en fosses circulaires et que dans certains cas ces dépôts d'animaux alternent avec des sépultures au sein d'une même structure, on peut avancer l'explication qu'il pourrait s'agir d'offrandes ou de dépôts de nature symbolique. Cet aspect est souligné par la présence de mobilier associé directement aux restes d'animaux comme sur le site de Hofstatt à Marlenheim où la disposition des restes du squelette d'un très jeune porcelet au contact direct d'un groupe de céramiques compose un dépôt remarquable. Sur le site d'Aue à Bruchsal, les découvertes d'ossements animaux s'organisent en différents ensembles, dont certains en association avec des restes humains ou dans leur proche voisinage (Behrends, 1988; Nickel, 1998). L'une de ces associations comprend une paire de chevilles osseuses d'aurochs disposée au-dessus d'un squelette humain ainsi que le squelette presque complet d'un porc domestique disposé au-dessus d'une sépulture multiple (structure 162) (Steppan, 1998). La dimension symbolique qui s'attache aux dépôts de carcasses complètes ou de parties de squelettes évoqués ci-dessus peut recouvrir d'autres types de restes osseux qui font très régulièrement partie du mobilier des fosses accueillant des inhumations comme les crânes d'animaux, le plus souvent disposés à l'envers signalés sur bon nombre de ces sites (Bruchsal, sépulture 5; Untergrombach, fosse 114; voir Nickel, 1998). Les bovins et les suidés semblent particulièrement bien représentés, c'est probablement dû à un intérêt et un traitement particuliers qui peuvent expliquer la préservation de ces pièces très fragiles et susceptibles d'être fragmentées du fait de l'exploitation bouchère.

La présence de squelettes entiers ou de parties de squelettes, l'association de pièces sélectionnées comme les crânes sont par ailleurs aussi des caractéristiques qui rapprochent ces vestiges découverts dans des secteurs domestiques de ceux associés aux enceintes du Michelsberg du Bassin parisien ou d'Allemagne du sud-ouest. Parmi le mobilier de la double enceinte de Maizy (Aisne), se distinguent des concentrations d'ossements entiers et en connexion de jeunes suidés, d'un jeune cerf, d'un nouveau-né de chevreuil et de mandibules isolées de putois, de blaireau et de castor et d'os long de chat sauvage, de martre et de castor. Sur les sites à fossés interrompus de la Bassée en Seine-et- 
Marne, a été soulignée la présence fréquente de crânes isolés (un crâne de porc à Gravon en F2, à Noyen en Fd 13), de segments anatomiques en connexion parmi des restes aux caractéristiques classiques de rejets culinaires (Noyen Fd 7, Fd 13, Fd 15, Gravon F2; Tresset, 1988 et 1996). Les vestiges osseux d'animaux associés aux fossés de l'enceinte de Hetzenberg à Neckarsulm-Obereisesheim (Beyer, 1970; Biel, 1990) correspondent à des squelettes ou des parties de squelettes parmi lesquels ont été dénombrés 6 bœufs et 8 moutons (dont quelques individus de quelques mois) et proviennent en grande majorité du fossé intérieur, d'un niveau situé au-dessus de dépôts humains. Les ensembles les plus importants se rapportent à des extrémités de moutons, une carcasse de mouton dont il manque les extrémités, plusieurs rachis de bouf domestique, une carcasse de bovin amputée d'un des membres postérieur et antérieur, deux bucranes de bœuf... L'étude de ces restes a souligné la rareté des traces de découpe et mis en évidence la possibilité de remontages entre des parties de squelettes déposées à différents endroits du fossé (Beyer, 1970), suggérant que ces dépôts ne participent pas forcément d'événements uniques mais bien plutôt d'une succession d'opérations disjointes dans le temps. La majorité des restes de faune se répartit dans le fossé interne avec comme exception notable la découverte dans le fossé intermédiaire d'un ensemble de 11 mandibules de bœuf, provenant d'au moins 8 individus (Beyer, 1970). L'ensemble de faune, assez conséquent, issu des fossés du site d'Aue à Bruchsal, présente, entre autres, comme particularité d'intégrer de nombreux restes d'aurochs dont la distribution anatomique fait apparaître une nette surreprésentation des parties crâniennes (Steppan, 2003). Cette caractéristique concerne aussi les suidés et les bovins domestiques. La disposition préférentielle de nombreux bucranes près des interruptions des fossés a été soulignée (Behrends, 1998). Plus de la moitié (environ $60 \%$ ) des ossements d'animaux issus de ce site se rapportent à des parties de squelettes en connexion d'animaux domestiques et sauvages : les tronçons de colonnes vertébrales et des extrémités des membres se rapportant à la plupart à des animaux sauvages et domestiques (bœufs, porcs, renards, aurochs, cerfs, chevreuils) semblent les plus fréquents.

Ces dépôts associés aux fossés d'enceinte recouvrent une grande diversité de traitement des os animaux à laquelle semble faire pendant une variabilité non moins importante des restes humains qui sont assez régulièrement découverts dans les mêmes structures et attestés sous forme de parties de squelettes, de restes isolés et dans certains cas fragmentés. Cependant, comme dans le cas des dépôts en fosses, aucune relation directe ne peut être établie entre les ossements humains et les restes animaux dont l'origine ne revêt pas de fait un caractère funéraire. L'existence, sur le site de Mairy (Ardennes), d'importants dépôts de carcasses animales qui s'organisent de façon complètement disjointe des sépultures, pourtant installées à proximité, souligne l'indépendance des deux formes de pratique. Les importants lots de faune recueillis sur ce site se répartissent entre, d'une part, des fosses-silos et, d'autre part, des épandages d'ossements situés à proximité des palissades associées au fossé (Arbogast, 1989 et 1998). Ces épandages sont formés de quelques squelettes plus ou moins complets mais surtout de portions de squelette en connexion, rachis de porc, membres de petits ruminants, colonne vertébrale de bovins. Ils ne subsistent en tant que tels que dans certains secteurs circonscrits, de quelques mètres de longueur, qui alternent avec des zones moins denses dans lesquelles ne subsistent que des vestiges épars qui pourraient correspondre à des vestiges de dépôts plus importants qui auraient été déplacés pour être enfouis dans les fosses-silos après une phase de décomposition. Cette hypothèse permet de rendre compte des caractéristiques très particulières par lesquelles se signalent les dépôts d'ossements d'animaux contenus dans les fosses-silos (Arbogast, 1998) :

- la variabilité qui caractérise les lots de faune associés à chacune des fosses-silos qui seraient constitués de portions des dépôts;

- la préservation de certaines connexions anatomiques et les nombreuses possibilités de restitutions articulaires en relation avec la présence d'ensembles anatomiques plus ou moins complets dans la zone des palissades;

- l'étroite imbrication des restes d'un grand nombre d'animaux qui marque le remplissage de certaines fossessilos et qui ne peut s'expliquer que par un décharnement préalable.

La possibilité d'une collecte des éléments de carcasses déposées près du fossé laisse par ailleurs supposer une décomposition à l'air libre, ou du moins en espace non colmaté et accessible, dont témoignent certaines traces d'érosion (desquamation attribuable aux effets de l'exposition à l'air libre) observées sur des os provenant des fosses ainsi que de nombreuses marques rapportables à des escargots carnivores (zonitidés) qui interviennent sur des os non enfouis et qui s'expliquent difficilement dans le cadre d'un enfouissement direct dans les silos. La présence des restes humains est strictement circonscrite aux deux sépultures individuelles implantées dans ce même secteur des palissades, au voisinage de ces épandages d'ossements animaux 
sans qu'il soit possible de préciser, dans l'état actuel de la documentation, s'il existe un lien entre ces structures (Marolle, 1989).

$\mathrm{Au}$ sein du corpus des dépôts d'animaux attestés en contexte Michelsberg ou Munzingen se dessinent les contours d'une pratique qui se décline sous différentes formes et à différents degrés d'intensité mais dont certaines caractéristiques apparaissent de façon récurrente. Ces dépôts impliquent des dépouilles complètes ou des segments assez importants de carcasses, d'animaux domestiques et sauvages, dont l'exploitation économique est soit absente, soit très peu intensive. L'organisation de ces dépôts en ensembles bien individualisés et stratigraphiquement distincts des sépultures avec lesquelles ils voisinent, parfois au sein d'une même structure, suggère qu'ils ne revêtent pas un caractère strictement funéraire. L'idée d'un fonctionnement symbolique s'appuie sur différents indices qui laissent soupçonner que la formation de tels assemblages a pu procéder d'une succession d'interventions disjointes dans le temps et s'inscrit dans une séquence de manipulations dont certaines se déroulent ou se continuent au-delà du dépôt des squelettes d'animaux et dont la finalité - cérémonielle ? cultuelle ? - nous échappe, de même que leur articulation avec les sépultures. Ces caractéristiques semblent partagées par un certain nombre de sites de la sphère chasséenne, dans le nord de la France où les dépôts d'animaux du fossé de Boury-en-Vexin (Oise) (Méniel, 1987) apparaissent assez analogues à ceux de Mairy, de même que dans le domaine méridional où, à l'occasion de fouilles de nombreuses inhumations en fosses, a pu être souligné le statut particulier de certains ensembles d'ossements d'animaux (Beeching, Crubézy, 1998) sans qu'il soit possible de développer ces comparaisons en raison du faible nombre de données publiées.

\section{LES INHUMATIONS DU SUD DE LA PLAINE DU RHIN SUPÉRIEUR DANS LE CADRE DE LA PROVINCE DES TOMBES EN FOSSES CIRCULAIRES}

Dans le Chasséen, les inhumations en fosses rondes ne représentent qu'une facette des nombreux rituels funéraires qui caractérisent cette culture (Lange, Poulain, 1984; Crubézy, 1991; Debut, Masset, 1991; Boujot et al., 1991; Beeching, Crubézy, 1998; Beeching, 2003; Beyneix, 2003). C'est également le cas dans la culture de Baalberg qui a livré de nombreuses petites nécropoles où les individus sont inhumés selon des règles strictes (Preuss, 1966; Kubenz, 1994). C'est avant tout l'absence de nécropole et l'extrême rareté des inhumations autres qu'en fosses rondes qui caractérisent l'aire Michelsberg/Munzingen, même si quelques indices nous montrent que parallèlement à cette pratique, existent d'autres sépultures régulières, aujourd'hui trop peu nombreuses pour que l'on puisse tenter d'en définir une norme. Cette situation est comparable à celle rencontrée en BasseBavière, dans la culture contemporaine de Münchshöfen où l'on ne connaît, à une exception près (Böhm, Schmotz, 1991), que des inhumations en habitat, soit dans de grandes fosses d'extraction, soit dans des fosses rondes (Böhm, 1981; Böhm, Pielmeier, 1993; Kreiner, 1995).

Il est frappant de constater qu'au niveau des gestes mortuaires, tous les aspects reconnus dans notre domaine d'étude se rencontrent à l'identique d'une extrémité à l'autre de la province funéraire des tombes en fosses circulaires. Ce constat vaut pour les positions affectées par les individus, pour les caractéristiques des tombes multiples, le rythme des dépôts (tombes à étages), ou encore pour les indices de manipulations ou les dépôts secondaires.

Les sépultures plurielles chasséennes de Saint-PaulTrois-Châteaux (fosse 69) et du Gournier à Montélimar (fosse EDF6) ont déjà été analysées sous l'angle de l'accompagnement (Boulestin, 2008; Jeunesse, 2010). Nous nous contenterons donc de souligner que ces deux ensembles sont strictement comparables à ceux de Didenheim ou de Bruchsal (Nickel, 1998) et qu'ils offrent de grandes similitudes avec plusieurs inhumations multiples Münchshöfen et Baalberg.

Pour la première de ces cultures, on retiendra une fosse d'habitat de Grossmehring (Schröter, 1996) qui a livré les restes de six individus dont un adulte masculin couché sur le dos, jambes fléchies, et cinq individus en procubitus parmi lesquels figurent un homme, une femme, un enfant et deux nouveau-nés. L'asymétrie est encore renforcée par le dépôt d'une bouteille au contact de l'adulte inhumé sur le dos. Il est difficile de ne pas reconnaître ici les traits qui signalent l'accompagnement dans les ensembles chasséens cités, qu'il s'agisse de l'inhumation sur le ventre ou du dépôt de mobilier à proximité de l'inhumation principale (Beeching, Crubézy, 1998). Peut-être faut-il également citer la double inhumation de Straubing qui associait, au sein d'un creusement de type silo, deux individus adultes dont un fléchi sur le côté et un déposé sur le dos (Böhm, Pielmeier, 1993). Pour la culture d'Altheim qui succède à celle de Münchshöfen, nous disposons de la fosse II du site d'Inningen, fouillée dans les années 1930, qui recelait six squelettes imbriqués dont un seul, le dernier déposé, était en position fléchie, les autres gisant en position désordon- 
née; des traces de violences ont également été remarquées sur plusieurs crânes (Maier, 1965).

Le corpus des restes humains en fosses rondes de la culture de Baalberg est relativement étoffé mais il s'agit, pour l'essentiel, d'ensembles anciennement fouillés et parfois documentés de façon sommaire. On peut néanmoins suspecter la pratique de l'accompagnement dans plusieurs fosses circulaires, notamment à Rossleben où un des niveaux d'inhumation a livré deux adultes déposés simultanément: l'un d'eux en position fléchie, l'autre couché sur le dos et présentant de surcroît un important traumatisme crânien (Preuss, 1966). On peut également retenir la fosse de Gehofen qui a livré six individus (deux adultes et quatre enfants) : la position des enfants n'est pas connue mais l'un des adultes était en position repliée et l'autre gisait sur le ventre (ibid.). Enfin, la fosse 27 du site de Weissenfels, site ayant livré huit fosses rondes contenant des restes humains, recelait les restes de quatre individus répartis sur trois niveaux; au niveau inférieur gisaient un adulte en position fléchi, orienté est-ouest, ce qui correspond à la norme des inhumations régulières en nécropole, et un enfant en position désordonnée. Deux autres niveaux d'inhumation recelaient respectivement un enfant et un adulte, orientés nord-sud et déposés sur le ventre. D’autres inhumations multiples, peu documentées, sont signalées à Pirkau (quatre individus et des traces de violences), Wildschütz et Preusslitz (Preuss, 1966).

Les dépôts secondaires sont bien attestés dans les ensembles Baalberg: on doit souligner la fréquence des dépôts de crânes, par exemple à Wansleben (Fischer, 1956), Obermörlen, Weissenfels, Pirkau, Braunsdorf et KlosterGröningen (Preuss, 1966). Des segments de squelettes infracrâniens épars sont signalés sur la majorité des sites.

Les manipulations de corps in situ sont plus difficiles à identifier: cela semble néanmoins être le cas à Kleinalsleben où l'on observe un squelette acéphale; à Wildschütz où il semble que les squelettes des trois individus inhumés aient été fortement remaniés (Preuss, 1966) ainsi qu'à Erfurt-Melchendorf (Bahn, 1989), site ayant livré une fosse contenant les restes partiellement déconnectés d'un adulte dont le crâne a de surcroît été prélevé. Les auteurs de la fouille hésitent cependant entre un dépôt secondaire et une manipulation intervenue in situ sur un cadavre en partie décomposé; la conservation de certains segments en position anatomique, notamment le bras et la main droite, pourrait plaider en faveur de la première hypothèse. En contexte Münchshöfen, nous renvoyons à la sépulture multiple de Grossmehring, déjà mentionnée, et pour laquelle les auteurs de la fouille signalent des déplacements d'ossements sur tous les individus inhumés: dans deux cas, il s'agit d'un déplacement du crâne, repositionné à distance, et dans un cas, de la manipulation des membres inférieurs de l'individu déposé sur le dos.

Enfin, le dernier point que nous voudrions souligner réside dans l'existence de creusements accueillant uniquement des corps en position désordonnée: c'est notamment le cas à Zauschwitz où deux niveaux d'inhumation ont respectivement livré un et deux individus en procubitus. Les fouilleurs évoquent l'hypothèse de corps jetés depuis l'ouverture de la fosse (Coblenz, Fritsche, 1973). Cette configuration n'est pas sans rappeler celle des individus isolés du corpus du Rhin supérieur - notamment l'inhumation 23 de Colmar - et suscite les mêmes interrogations quant à leur statut.

Les analogies avec le corpus du sud de la plaine du Rhin supérieur sont évidentes et il paraît inutile d'insister davantage sur la communauté de pratiques qui caractérise les groupes observant l'inhumation en fosses rondes, particulièrement les cultures de Baalberg et de Michelsberg dont les liens ont déjà été soulignés (Jeunesse et al., 2003). Il nous semble que la grille de lecture proposée pour les inhumations en fosses rondes du Michelsberg, du Munzingen ou du Chasséen peut, sans trop de difficultés, être transposée aux corpus des cultures plus orientales. Les interprétations proposées par les chercheurs d'Europe centrale sont cependant très éloignées de celles que nous retenons ici et qui intègrent l'ensemble des données au sein d'un système structuré. Dans les publications d'Allemagne orientale, les inhumations en fosses rondes sont parfois assimilées à des fosses à offrande (Opfergruben ou Depositgruben; Döhle et al., 1992), ou comme des structures trahissant des pratiques cultuelles fort complexes ${ }^{9}$ (Bahn, 1989), conception reposant sur une hypothèse assez proche de celle jadis soutenue

9. C'est en ce sens que sont interprétés, par exemple, les dépôts de Weissenfels, d'Erfurt-Melchendorf et Halle-Ammendorf. À nos yeux, ces dépôts ne diffèrent pas fondamentalement de ceux du sud de la plaine du Rhin et l'on y retrouve notamment, aux côtés des restes humains en dépôts secondaires ou des corps en position désordonnée, des individus en position régulière, souvent orientés selon la norme Baalberg. Les ossements humains mêlés à des restes d'animaux de Halle-Ammendorf ou de Weissenfels évoquent les restes en position secondaire du site Michelsberg d'Untergrombach découverts en contextes détritiques, et il ne paraît pas nécessaire d'invoquer l'existence de Kannibalmahlzeit (Kubenz, 1994). Dernier exemple, l'Opfergrube d'Alsleben (Döhle et al., 1992) ayant livré une couche inférieure riche en tessons et en ossements d'animaux est interprétée comme fosse à offrande recelant les restes enfouis d'un sacrifice (Opfermahlzeit), là où nous ne voyons pour notre part qu'une couche détritique. 
par J. Lichardus (Lichardus, 1986) qui, nous l'avons déjà mentionné, considérait que les éléments présents à tous les niveaux des creusements utilisés comme fosses sépulcrales, et notamment ce que nous considérons ici comme des couches détritiques, participaient du rituel funéraire.

Cette contribution à l'étude des dépôts en fosses circulaires du Néolithique récent soulève certainement davantage d'interrogations qu'elle n'apporte de réponses. Les avancées qui nous paraissent les plus significatives pour notre région d'étude sont celles ayant trait à la définition d'un système funéraire complexe mais cohérent, et à la mise en évidence de manipulations postdécompositionnelles intervenant sur les restes humains comme sur les restes d'animaux.

Le problème de l'origine de la tradition des inhumations en fosses circulaires n'est pas totalement résolu mais l'hypothèse d'une racine chasséenne et d'une introduction de cette pratique dans le sud de la plaine du Rhin par l'intermédiaire du Bischheim occidental du Rhin supérieur peut être posée.

Outre l'architecture des fosses sépulcrales dont nous avons souligné qu'il s'agissait le plus souvent de fosses de stockage désaffectées, la tradition funéraire du Néolithique récent régional s'illustre par la relative fréquence des sépultures multiples où figurent un individu fléchi en position conventionnelle, parfois accompagné de mobilier céramique ou sur matière dure animale, et un nombre variable d'individus en position désordonnée. Le principe d'asymétrie qui semble régir ce type de dépôt a récemment été interprété en termes d'accompagnement, hypothèse à laquelle l'analyse du riche corpus alsacien, nous amène à souscrire. Les cas de dépôts simultanés non asymétriques sont assez peu fréquents et associent systématiquement adultes et enfants en bas âge ou enfants entre eux, mais, le fait doit être souligné, jamais deux adultes. L'hypothèse d'une gestion collective, évoquée pour certains ensembles chasséens, ne repose aujourd'hui que sur un unique ensemble régional et reste une hypothèse à confirmer pour le sud de la plaine du Rhin. Les sépultures «à étages»y sont fréquentes mais l'intentionnalité du geste, bien que vraisemblable, ne peut être formellement démontrée. Seul l'ensemble de Leimen à Rosheim (st. 104) montre un cas de dépôts successifs où le second individu inhumé a été déposé alors que le premier inhumé, s'étant décomposé en espace non colmaté, était encore visible.

Les fouilles récentes, et tout particulièrement celle menée sur le site de l'Aérodrome à Colmar, ont permis de mettre clairement en évidence l'existence de réinterventions sur les restes humains et sur les dépôts d'animaux, dépôts dont nous retenons le caractère symbolique. Il s'agit soit du repositionnement dans la fosse, soit du prélèvement de segments anatomiques, gestuelle qui rappelle, à une échelle plus modeste, la gestion complexe des restes osseux observée dans les enceintes. Cette observation permet partiellement d'expliquer la présence dans les fosses circulaires de dépôts secondaires d'ossements humains sous forme de fragments isolés ou de parties anatomiques.

Enfin, l'hypothèse de dépôts humains réalisés en dehors du cadre funéraire nous est suggérée par l'ensemble 23 de Colmar où voisinent un individu en position non conventionnelle et deux colliers de perles en cuivre. L'idée d'une inhumation périphérique dépendante d'une inhumation principale localisée dans un autre creusement - variante du modèle de l'accompagnement - ne rend pas compte, à nos yeux, de cette improbable association. Nous sommes tout à fait conscients que l'hypothèse de dépôts non funéraires - hypothèse que l'on pourrait étendre aux nombreux individus isolés et en positions non conventionnelles des corpus Michelsberg/Munzingen et Baalberg - ne repose que sur de maigres données, suffisamment troublantes néanmoins pour nous amener à ne pas écarter cette piste de recherche.

L'analyse des corpus des inhumations des différentes régions constituant la province funéraire des tombes en fosses circulaires récemment définie par C. Jeunesse (Jeunesse, 2010) permet de souligner la grande proximité des traditions mortuaires, qu'il s'agisse de la pratique de l'accompagnement que l'on retrouve du Chasséen au Baalberg, des manipulations postdécompositionnelles que l'on peut identifier sur plusieurs ensembles Münchshöfen et Baalberg, des dépôts d'animaux entiers et, peut-être, des dépôts humains non funéraires. 


\section{CATALOGUE}

\section{BAS-RHIN}

\section{ACHENHEIM (TUILERIE E. SCHAEFER)}

Bibliographie: Schaeffer, 1926; Eich-Francke, 1967; Lüning, 1968.

\section{Sépulture double de janvier 1922}

Inhumation de deux adultes dans une fosse circulaire (diam.: 1,70 m; prof.: 2,50 m) ; une femme (35/60 ans) et un individu de sexe non déterminé (40/65 ans).

Position: «assis» (comprendre probablement «fléchis»). Orientation: non renseignée.

Mobilier: plusieurs tessons, fragments d'os animaux et fragments de torchis (mobilier détritique).

Datation: Michelsberg.

\section{Sépulture de février 1922}

Inhumation d'un enfant (12/15 ans) dans une fosse circulaire (diam. : 1,30 m; prof.: $1,50 \mathrm{~m}$ ).

Position: d'après Schaeffer, l'individu reposait «assis», membres inférieurs fléchis ramenés vers le corps et membres supérieurs repliés.

Orientation: non renseignée.

Mobilier: tessons, une lame de hache dans une gaine en bois de cerf, fragments d'os animaux et fragments de torchis (mobilier détritique).

Datation: Michelsberg.

\section{Sépulture de mars 1924}

Inhumation d'un adulte de sexe masculin (45/75 ans) dans une fosse circulaire (diam.: 0,70 m ; prof. : 1,20 m). Position: membres supérieurs repliés et membres inférieurs fléchis. «La position des jambes par rapport au crâne indiquait clairement que le mort avait été placé dans la fosse la tête en bas» (Schaeffer, 1925-1926).

Orientation: non renseignée.

Mobilier: tessons, une lame de silex et fragments d'os animaux (mobilier détritique).

Datation: Néolithique récent probable.

\section{ECKBOLSHEIM (PARC D'ACTIVITÉS)}

Bibliographie: Henigfeld, 2005.

\section{Sépulture 412}

Inhumation d'un adulte de sexe masculin (30/49 ans) dans une fosse quadrangulaire aux angles arrondis, ajustée à la taille du corps (dim. : 1,68 m x 0,95 m).

Position: le corps repose sur le flanc gauche, membres inférieurs fléchis à gauche, membre supérieur droit ali- gné le long du corps et avant-bras gauche fléchi. L'étude taphonomique conclue à un colmatage immédiat.

Orientation: est-ouest.

Mobilier: aucun.

Datation: BORS d'après une datation radiocarbone (GrN-30114, $5380 \mathrm{BP} \pm 40$ ans, soit 4330-4170 av. J.-C. à $1 \sigma)$.

\section{ENTZHEIM (BASE AÉRIENNE)}

Bibliographie: Sauer, 1959; Lambach, 1986; Eich-Francke, 1967 ; Lüning, 1968.

\section{Fosse 1958}

Inhumation d'un enfant (environ 3 ans) découverte à 1,30 $\mathrm{m}$ de profondeur; creusement non renseigné.

Position et orientation: non renseignées.

Mobilier: tessons de 3 vases et 3 outils en os.

Datation: Munzingen B.

\section{ENTZHEIM (SABLIÈRE OESCH)}

Bibliographie: Schmitt, 1974; Lambach, 1986.

Inhumation d'un adulte de sexe masculin (35/65 ans) dans une fosse circulaire (diam.: 1,80 m env.; prof.: 0,40 m env.)

Position: individu couché sur le côté droit, membres inférieurs fléchis. La déconnexion du crâne, de l'atlas et de l'axis a amené F. Lambach à évoquer l'existence «d'une structure périssable qui aurait surélevé la tête lors de l'inhumation".

Orientation: nord-est/sud-ouest.

Mobilier: aucun.

Datation: éventuellement BORS d'après le contexte mais l'attribution au Michelsberg reste possible.

\section{ENTZHEIM (ZAC LES TERRES DE LA GHAPELlE 2)}

Bibliographie: Schneikert, 2006.

Le site, en cours de fouille au printemps 2009 (fouilles P. Lefranc, inédit), a livré une inhumation d'enfant orientée sud-est/nord-ouest, tête au sud-est, attribuée au Bischheim rhénan.

Mobilier: céramique décorée, perles en jais et en calcaire, coquillages, grattoir en silex.

St. 30

Inhumation mal conservée d'un individu.

Position: non renseignée.

Orientation: sud-est/nord-ouest, tête au sud-est. 
Mobilier: lame de hache polie à talon pointu et piqueté. Datation: Bischheim ou épi-Roessen.

\section{ENTZHEIM-GEISPOLSHEIM (AÉROPARC)}

Bibliographie: Croutsch et al., 2007; Croutsch et al., 2008. St. 1511

Ossements d'enfant isolés dans une fosse circulaire (diam.: 1,20 m; prof.: 0,36 m).

Mobilier: 1 vase, 1 éclat de silex et 2 éclats de lames polies de hache.

Datation: Michelsberg III.

\section{St. 1183}

Inhumation d'un enfant (12/18 mois) dans une fosse ovale (diam.: 1,80 m; prof.: 1,10 m). Le sujet repose au sein du comblement à $0,50 \mathrm{~m}$ sous le niveau de décapage, dans une couche riche en charbon de bois et en torchis. Position: sur le côté droit, membres inférieurs fléchis.

Orientation: nord-est/sud-ouest.

Mobilier: aucun.

Datation: Michelsberg III d'après une datation radiocarbone (Poz-19242, $5130 \mathrm{BP} \pm 40$ ans, soit 39803810 av. J.-C. à 1 o).

\section{St. 1266A}

Inhumation de deux adultes de sexe féminin dont l'une âgée de 25/30 ans dans une fosse subcirculaire (dim.: $1 \mathrm{~m}$ x 1,20 m; prof.: $1 \mathrm{~m}$ ). Les deux individus reposent sur le fond. Les espaces de décomposition diffèrent ( $\mathrm{A}=$ espace vide; $\mathrm{B}$ = colmatage différé).

Position: amas d'ossements déconnectés mais conservant «une logique anatomique susceptible de témoigner d'un dépôt primaire» (individu A). Interprétation proposée: inhumation assise, «membres inférieurs fléchis en avant du corps, les membres supérieurs fléchis, les mains au contact l'une de l'autre». Décomposition en espace vide. L'éventualité d'une décomposition au sein d'un contenant de type coffre ayant limité l'ampleur des déplacements est évoquée. Dépôt postérieur à celui de l'individu B; sur le côté gauche en position fléchie. Décomposition en espace colmaté (individu B).

Orientation: nord-nord-ouest/sud-sud-est (individu B).

Mobilier: un poinçon en os retrouvé à proximité de l'individu A; tessons dans le comblement.

Datation: Michelsberg III d'après une datation radiocarbone (Poz-19243, 5100 BP \pm 40 ans, soit 3970-3800 av. J.-C. à 1 o).

\section{St. 1411A}

Inhumation d'un adulte (de sexe féminin ?; individu A) et d'un enfant (B) en contact strict dans une fosse circulaire (diam.: 1,50 m; prof.: 0,42 m). Les squelettes reposent à une dizaine de centimètres au-dessus du fond. L'espace de décomposition n'a pas pu être déterminé Position: l'individu A en position fléchie, sur le côté droit; l'individu B reposant sur le côté gauche.

Orientation: nord-ouest/sud-est (A) et est-ouest (B).

Mobilier: nombreux fragments d'os animaux.

Datation: Michelsberg III d'après une datation radiocarbone (Poz-19246, 5090 BP \pm 40 ans, soit 3960-3800 av. J.-C. à 1 б).

\section{St. 1422}

Inhumation d'un adulte de sexe masculin dans une fosse subcirculaire (diam.: 1,55 m; prof.: 1,30 m). L'individu repose au sein du comblement. Décomposition probablement en espace colmaté.

Position: en position fléchie sur le côté droit.

Orientation: nord-ouest/sud-est.

Mobilier: un os long animal déposé en avant des cuisses. Datation: Michelsberg III d'après une datation radiocarbone (Poz-19195, $5110 \pm 40$ BP, soit 3970-3800 av. J.-C. à 1 o).

\section{St. 1467}

Inhumation d'un adulte de sexe masculin dans une fosse subcirculaire (diam.: 1,65 m; prof.: 0,42 m). Le squelette repose sur le fond.

Position: sur le dos avec un appui côté droit; membres inférieurs fléchis à droite. L'espace de décomposition n'a pas pu être déterminé.

Orientation: sud/nord.

Mobilier: dépôt d'un canidé à l'aplomb et à une quarantaine de centimètres au-dessus du crâne.

Datation: Michelsberg IV ou Munzingen B d'après une datation radiocarbone (Poz-19196, $5110 \mathrm{BP} \pm 40$ ans, soit 3770-3660 av. J.-C. à 1 б).

\section{ERGERSHEIM}

Bibliographie: Lambach, 1986.

\section{Fosse 1914}

Inhumation d'un adulte de sexe masculin (45/70 ans) découverte en 1914; creusement non renseigné.

Position et orientation: non renseignées.

Mobilier: quelques tessons.

Datation: Néolithique récent probable.

\section{GEISPOLSHEIM (BRUECHEL)}

Bibliographie: Jeunesse et Sainty, 1986.

\section{Fosse 14}

Un fragment d'épiphyse d'humérus d'adulte en contexte détritique dans une fosse contenant un abondant mobilier détritique.

Datation: Munzingen B. 
9. GEISPOLSHEIM (FORLEN)

Bibliographie: Billoin et al., 2005.

St. 105

Inhumation d'un adulte de sexe féminin (+ de 30 ans) dans une fosse ovale (dim.: 1,35 m x 1,18 m; prof.: $0,15 \mathrm{~m})$.

Position: sur le dos, fémurs en forte flexion vers la droite, les «membres supérieurs en élévation, placés vers la gauche du corps».

Orientation: nord-est/sud-ouest.

Mobilier: quelques tessons dans le remplissage.

Datation: Michelsberg III/IV ou Munzingen B d'après une datation radiocarbone (Eth-28520, $4975 \mathrm{BP} \pm 55$ ans, soit 3900-3660 av. J.-C. à $1 \sigma$ ).

\section{GOUGENHEIM}

Bibliographie: inédit; diagnostic sur le tracé de la future Ligne à Grande Vitesse Est. Trois inhumations en silo dont deux fouillées. Contexte Michelsberg/Munzingen. Fouilles en cours.

\section{ID 34}

Inhumation d'un individu adulte dans une fosse circulaire. Position: sur le côté gauche, membres inférieurs fléchis. Orientation: sud-nord.

Mobilier: quelques tessons dans le remplissage.

Datation: Néolithique récent probable.

ID 52

Inhumation d'un adulte dans une fosse circulaire.

Position: sur le dos, membres inférieurs en extension dessinant un angle de $90^{\circ}$ avec le tronc.

Orientation: tête au nord-nord-ouest.

Mobilier: quelques tessons dans le remplissage.

Datation: Néolithique récent.

\section{HOHATZENHEIM (UNTEN IM KUGLER)}

Bibliographie: Gallay, 1970; Stieber, 1956.

Inhumation d'un adulte dans une fosse de type «fente" (dim.: 2,20 m x 0,60 m; prof. : 1,60 m).

Position: couché sur le ventre, membres inférieurs en extension, bras repliés ramenés devant le visage.

Orientation: est-ouest.

Mobilier: gobelet complet déposé sous la main gauche, près de la poitrine.

Datation: Néolithique récent moyen ou récent.

12. HOLTZHEIM (ALTMATT, LES ABATTOIRS ET AM SCHLUESSELBERG)

Bibliographie: Kühnle et al., 1999-2000; Lefranc, 2001.

\section{Les Abattoirs 158}

Inhumation/dépôt secondaire d'un adulte de sexe masculin (+ de 20 ans) dans un chablis.

Position: squelette incomplet sans ordre anatomique. Absence des os correspondant aux connexions labiles.

Mobilier: aucun.

Datation: Néolithique récent probable.

\section{Altmatt 88}

Inhumation d'un adulte dans une fosse subrectangulaire aux angles arrondis (long.: $3 \mathrm{~m}$; larg.: 1,10 m; prof. : 0,10 m).

Position: allongé sur le dos, membres supérieurs le long du corps et membres inférieurs en extension.

Orientation: est-sud-est/ouest-nord-ouest.

Mobilier: aucun.

Datation: Michelsberg III d'après une datation radiocarbone (Lyon-10495: $5085 \mathrm{BP} \pm 40$ ans, soit 39603800 av. J.-C. à $1 \sigma)$.

\section{Altmatt 260}

Inhumation d'un adulte dans une fosse circulaire (diam. : 1,14 m; prof.: 1,30 m).

Position: couché sur le flanc gauche, membres inférieurs fléchis et bras ramenés vers le visage.

Orientation: ouest-est.

Mobilier: un tesson issu du comblement.

Datation: Michelsberg probable.

\section{Am Schluesselberg 79}

Inhumation triple; deux squelettes (sujets 1 et 2) et un bloc craniofacial (sujet 3) reposant sur deux niveaux distincts dans une fosse circulaire partiellement comblée avant le dépôt des corps (diam.: 1,90 m; prof.: 0,70 m).

Position: sur le côté droit, bras alignés le long du corps et avant-bras fléchis, cuisses en extension et jambes légèrement fléchies (sujet 1); sur le dos, membres en extension (sujet 2); calvarium isolé (sujet 3) placé au même niveau que le sujet 2 .

Orientation: est-ouest (sujet 1) et sud-est/nord-ouest (sujet 2). Mobilier: un éclat de silex et un collier composé de perles cylindriques en calcaire de coquillages et d'un galet perforé (individu 2).

Datation: Michelsberg III/IV ou Munzingen B d'après une datation radiocarbone (Lyon-8263: $4990 \mathrm{BP} \pm 50$ ans, soit 3920-3700 av. J.-C. à $1 \sigma)$.

\section{Holtzheim (SABLiÈres RÉUNIES)}

Bibliographie: Lasserre et al., 1999.

Les restes humains (corps en connexion, os erratiques/ dépôts secondaires et les os brûlés (incinération ?) pro- 
viennent des segments I, III et IV d'un fossé interrompu appartenant probablement à un monument de type «Rosheim».

Datation: le dépôt 1 , BORS d'après une datation radiocarbone (Gra-13966: $5340 \mathrm{BP} \pm 40$ ans, soit 42604060 av. J.-C. à 1 o) ; le dépôt 2, Bruebach-Oberbergen ou BORS d'après une datation radiocarbone (Gra-13972: $5390 \mathrm{BP} \pm 40$ ans, soit 4330-4180 av. J.-C. à 1 o) ; «l'incinération ", Bruebach-Oberbergen ou BORS d'après une datation radiocarbone (Gra-13972: $5390 \mathrm{BP} \pm 80$ ans, soit 4340-4070 av. J.-C. à 1 o).

\section{INGENHEIM (HUNGERBERG)}

Bibliographie: inédit; diagnostic sur le tracé de la future Ligne à Grande Vitesse Est.

St. 94

Inhumation en fosse circulaire d'un individu reposant à mi-hauteur d'une fosse circulaire.

Position: sur le dos. La position des membres inférieurs, détruits lors du sondage mécanique, n'est pas connue.

Orientation: non renseignée.

Mobilier: 2 morceaux d'ocre, 1 meule en grès et 1 tesson dans le remplissage. La fosse contenait également une importante quantité de torchis rubéfié.

Datation: Michelsberg III/IV.

\section{ITTENHEIM (COMPLEXE SPORTIF)}

Bibliographie: Lefranc et al., 2009.

SP 33

Inhumation d'un enfant (4/6 ans). Limite de creusement non visible.

Position: sur le dos, les bras alignés le long du corps, les membres inférieurs en extension.

Orientation: sud-sud-est/nord-nord-ouest.

Mobilier: fragments d'une hache en bois de cerf perforée et d'un andouiller de cerf perforé.

Datation: Bruebach-Oberbergen d'après une datation radiocarbone (Poz-19068: $5420 \mathrm{BP} \pm 40$ ans, soit 43354255 av. J.-C. à $1 \sigma)$.

\section{LINGOLSHEIM (SABLIÈRE LIENHARDT)}

Bibliographie: Forrer, 1938; Eich-Francke, 1967; Lüning, 1968; Lichardus-Itten, 1980.

SP 38

Inhumation d'un adulte; creusement non renseigné.

Position: sur le dos.

Orientation: sud-est-nord-ouest.

\author{
Mobilier: aucun. \\ Datation: Néolithique récent? \\ SP 39
}

Inhumation double avec adultes de sexe masculin superposés (20/45 ans); creusement non renseigné.

Position: le squelette supérieur, couché sur le côté droit en position fléchie; le squelette inférieur, couché sur le dos

Orientation: sud-est-nord-ouest.

Mobilier: tessons et 2 poinçons en os.

Datation: Néolithique récent, éventuellement Munzingen d'après le fragment de vase publié par R. Forrer.

\section{MARLENHEIM (CONTOURNEMENT ROUTIER)}

Bibliographie: Tristan, 2004.

St. 3048

Inhumation d'un adulte de sexe masculin (?; + de 19 ans) dans une fosse ovale (dim.: 0,80 m x 0,70 m; prof. : 0,20 m).

Position: en partie sur le ventre, membres inférieurs fléchis à droite, avant-bras fléchis en avant du thorax.

Orientation: nord-ouest-sud-est.

Mobilier: un vase.

Datation: Michelsberg IV.

\section{MARLENHEIM (HOFSTATT)}

Bibliographie: Châtelet, 2009.

Le site a livré, outre les trois structures circulaires contenant des restes humains, un dépôt installé dans une petite niche aménagée en sape dans le fond d'un silo et contenant cinq gobelets complets datables du Michelsberg III ainsi que les restes d'un jeune suidé.

\section{St. 109}

Inhumation quadruple dans une fosse circulaire (diam. : 1,25 m ; prof. : 0,80 m). À environ 0,50 m du fond, dépôt d'un adulte et de deux enfants en contact strict et restes partiels d'un quatrième individu représenté par un crâne et quelques ossements (remaniement ou dépôt secondaire).

Position: un adulte déposé en position fléchie sur le côté gauche; les deux enfants en position désordonnée.

Orientation: nord-est-sud-ouest (adulte).

Mobilier: un grand fragment de vase brisé en deux parties dont une reposait sur le bassin de l'adulte; sur le fond du creusement: fragments de meules et quelques restes de faune.

Datation: Michelsberg III. 


\section{St. 649}

Dépôt secondaire en fosse circulaire (diam.: 1,70 m; prof. : $0,15 \mathrm{~m})$.

\section{St. 650}

Inhumation en fosse circulaire (diam.: 1,50 m; prof.: $0,45 \mathrm{~m}$ ) de deux adultes (individus 1 et 2) et des restes d'un troisième individu. Ce dernier n'est représenté que par son bloc craniofacial reposant en contact avec l'épaule de l'individu 2. Celui-ci est également incomplet. Seule la moitié supérieure du corps a été inhumée dans cette fosse, à $5 \mathrm{~cm}$ environ sous le niveau de l'individu 1. Le prélèvement probable de la moitié inférieure du corps de l'adulte 2 intervient avant le dépôt du $\mathrm{n}^{\circ} 1$.

Position: en position fléchie sur le côté gauche (individu 1); déposé sur le flanc gauche (individu 2).

Orientation: nord-ouest/sud-est (individu 1) ; ouest-est (individu 2).

Mobilier: néant.

Datation: Néolithique récent?

\section{MARLENHEIM (Lotissement LA PeUpleraie)} Bibliographie: Châtelet, 2002.

\section{«Tombe néolithique»}

Inhumation d'un adulte dans une fosse ovale ajustée aux dimensions du corps (dimensions non renseignées). Position: allongé sur le dos, les bras alignés le long du corps et les membres inférieurs en extension.

Orientation: nord-nord-ouest/sud-sud-est.

Mobilier: une armature de faucille en silex retrouvé à «l'emplacement de la main droite».

Datation: Michelsberg III/IV ou Munzingen B d'après une datation radiocarbone (Gr-?: $5000 \mathrm{BP} \pm 110$ ans, soit 3950-3690 av. J.-C. à 1 б).

\section{MATZENHEIM (LE LAVOIR)}

Bibliographie: Toullec, 2008.

St. 270

Inhumation d'un individu adulte (20/49 ans) déposé dans une fosse quadrangulaire au niveau du décapage, devenant ovale au niveau du corps (dim.: $2 \mathrm{~m} \mathrm{x} \mathrm{1,55} \mathrm{m}$ au niveau du décapage à 2,60 $\mathrm{m}$ x $1,80 \mathrm{~m}$ au niveau du corps ; prof.: 0,70 m).

Position: allongé sur le dos; le membre supérieur gauche est aligné le long du corps, le droit fléchi; les membres inférieurs en extension, croisés à hauteur des jambes. Orientation: ouest-nord-ouest/est-sud-est.

Mobilier: un gobelet et une bouteille déposés aux pieds ainsi que deux artefacts de silex retrouvés dans et à côté de sa main gauche.

Datation: Michelsberg ancien du Rhin supérieur d'après le mobilier céramique et une datation radiocarbone (Eth36782: $5185 \mathrm{BP} \pm 40$ ans, soit 4040-3960 av. J.-C. à 1 б).

\section{MitTelhaUSBERGEN}

Bibliographie: Gallay, 1970.

MAS 33928

Un individu fortement contracté; aucune autre donnée. Datation: Néolithique récent?

\section{MUNDOLSHEIM (HAUSBERGEN)}

Bibliographie: Forrer, 1912; Lambach, 1986.

Fosse 1 (MAS 17795)

Inhumation de trois enfants (dont un âgé de 7/9 ans) dans une fosse circulaire (diam. : 0,60 m; prof. : 0,90 m). Position: d'après les ossements du seul individu conservé, étudiés par F. Lambach, les membres inférieurs et supérieurs sont repliés (concrétions unissant les éléments).

Orientation: non renseignée.

Mobilier: aucun.

Datation: Néolithique récent?

Fosse 21 A (MAS 8076)

Inhumation d'un jeune adulte de sexe masculin (16/17 ans) ; creusement non renseigné.

Position: «... couché sur le ventre... La moitié inférieure du corps (bassin et membres inférieurs) est en forte rotation gauche» (Lambach, 1986).

Orientation: non renseignée.

Mobilier: quelques fragments de faune et deux tessons. Datation: Néolithique récent?

\section{Fosse 41 A (MAS 15946)}

Inhumation d'un adulte de sexe féminin (20/21 ans) dans une fosse circulaire (diam. 1,20 m; prof. : 1,20 m). Position: sur le côté droit, membres inférieurs fléchis. Orientation: non renseignée.

Mobilier: quelques tessons.

Datation: Néolithique moyen ou récent.

Fosse 43 (MAS 15947)

Inhumation d'un adulte de sexe masculin (25/45 ans) dans une fosse circulaire (diam. : 1,20 m; prof. : 1,10 m). Position: «en décubitus dorsal... Les cuisses sont écartées et les jambes sont repliées sur la face postérieure des cuisses, en flexion forcée. Les bras sont étendus le long du corps; les avant-bras sont en flexion forcée sur les bras» (ibid.). 
Orientation: non renseignée.

Mobilier: un tesson.

Datation: Néolithique récent.

\section{MAS 15948}

Un crâne d'enfant venant d'une sépulture détruite (env.

12 ans); creusement non renseigné.

Position: non renseignée.

Orientation: non renseignée.

Mobilier: non renseigné.

Datation: Néolithique récent?

\section{MAS 2804}

Crâne et mandibule d'un adulte (25/55 ans); creusement non renseigné.

Position: non renseignée.

Orientation: non renseignée.

Mobilier: aucun.

Datation: Néolithique récent?

Remarque: enfoncement de l'arrière-crâne.

\section{REICHSTETT (RMS)}

Bibliographie: Blaizot, 2001.

\section{Fosse 19}

Inhumation de deux adultes, l'un de sexe masculin (individu 1), l'autre de sexe féminin (individu 2) dans une fosse circulaire (diam. : $1 \mathrm{~m}$; prof. : 0,87 m). Dépôt simultané au sein du remplissage, à $0,47 \mathrm{~m}$ au-dessus du fond de la fosse.

Position: sur le dos, inorganisée, jambes disjointes (individu 1); sur le côté gauche, membres inférieurs fléchis (individu 2).

Orientation: nord-est/sud-ouest (individu 1) et nordouest/sud-est (individu 2).

Mobilier: tessons et un éclat de quartzite.

Datation: Néolithique récent.

\section{Fosse 102}

Inhumation d'un adulte de sexe masculin en fosse circulaire (diam.: 1,30 m; prof.: 0,50 m). La décomposition s'est effectuée en espace ouvert.

Position: sur le côté droit, membres inférieurs fléchis. Bras ramenés vers le haut du corps.

Orientation: ouest-est.

Mobilier: une jatte incomplète et un grand fragment de bouteille.

Datation: Munzingen $\mathrm{C}$ d'après une datation radiocarbone (Ly-9198: 4825 BP \pm 35 ans, soit 3660-3530 av. J.-C. à $1 \sigma)$.

Fosse 152

Inhumation d'un sujet immature (11/16 ans) dans une circulaire (diam.: $2 \mathrm{~m}$; prof.: 0,59 m).

Position: plaqué contre la paroi de la fosse. Couché sur le dos, membres inférieurs fléchis vers la droite (surélevés et appuyés sur la paroi de la fosse, avant-bras ramenés vers le haut du corps).

Orientation: nord-ouest/sud-est.

Mobilier: à $20 \mathrm{~cm}$ au-dessus du crâne, squelette d'un suidé immature (offrande ?).

Datation: Néolithique récent probable.

\section{ROSHEIM (LEIMEN)}

Bibliographie: Lefranc et al., 2007; Boës, 2007.

\section{St. 94}

Inhumation d'un adulte de sexe féminin dans une fosse de plan bilobé (long. : 1,70 m; larg. : 1,10 m; prof. : $1,85 \mathrm{~m})$. Le sujet repose à $0,75 \mathrm{~m}$ au-dessus du fond.

Position: fortement contractée. Couché sur le côté droit, bras ramenés vers le haut du corps, membres inférieurs fléchis vers la droite.

Orientation: sud-est/nord-ouest.

Mobilier: deux vases, dont un tulipiforme complet, déposés aux pieds ainsi que quelques fragments de torchis, des tessons et de la faune retrouvés dans le remplissage au-dessous du défunt.

Datation: Michelsberg III.

\section{St. 104}

Inhumation d'un adulte de sexe féminin (individu 1), d'un immature de sexe masculin entre 16 et 18 ans (individu 2) et d'un nourrisson (individu 3) dans une fosse circulaire (diam. : 1,10 m; prof. : 1,46 m). Les sujets reposent à environ $20 \mathrm{~cm}$ au-dessus du fond du creusement. Les dépôts sont très probablement successifs (individus 2 et 3 , puis individu 1).

Position: sur le côté droit, bras ramenés vers le haut du corps, membres inférieurs fléchis vers la droite. Espace de décomposition : colmaté (individu 1); sur le dos, bras ramenés sur les épaules, membres inférieurs fléchis vers la droite. Position originelle: genoux surélevés et pieds plaqués à la verticale contre la paroi. Espace de décomposition : ouvert (individu 2); sur le ventre, membres inférieurs fléchis sous le corps (individu 3).

Orientation: nord-est/sud-ouest (individu 1) ; est-ouest (individu 2); sud-ouest/nord-est (individu 3).

Mobilier: le remplissage sous-jacent aux inhumations a livré un fragment de meule et un fragment de molette. Datation: Michelsberg III d'après une datation radiocarbone (GRA31501: $5100 \mathrm{BP} \pm 35$ ans, soit 39603800 av. J.-C. à 1 o). 


\section{St. 107}

Inhumation d'un enfant en fosse circulaire (diam.: $1,20 \mathrm{~m}$; prof.: 0,69 m). Le sujet repose à une quinzaine de centimètres au-dessus du fond de la fosse, plaqué contre la paroi, sur une couche détritique.

Position: couché sur le flanc gauche, bras ramenés vers le visage, membres inférieurs probablement fléchis vers la gauche.

Orientation: nord-est/sud-ouest.

Mobilier: nombreux tessons et fragments de torchis provenant de la couche détritique sous-jacente à l'inhumation. Datation: Munzingen B.

Particularité: enfoncement circulaire à l'arrière du crâne. St. 154

Adulte de sexe masculin. Creusement: silo de type «Beutelförmige grube» (diam. : 1,34 m ; prof. : 1,70 m). L'individu repose sur le fond - concave - du creusement. Quelques tessons, un fragment de meule et plusieurs blocs de grès reposaient groupés au nord du squelette, dans le remplissage sus-jacent.

Position: sur le dos, bras gauche ramené vers le haut du corps, bras droit au niveau du ventre. Membres inférieurs fléchis légèrement déportés vers la droite. Le genou gauche repose sur l'hémi-thorax droit.

Orientation: sud-ouest/nord-est.

Datation: Michelsberg III d'après une datation radiocarbone (GRA31503: $5115 \mathrm{BP} \pm 35$ ans, soit 39703810 av. J.-C. à 1 б).

\section{St. 167}

Un fragment de diaphyse de fémur humain très érodé. La couche ayant livré cette diaphyse contenait également des restes de faune, du pisé, des tessons de céramique et de l'outillage lithique et osseux. Le mobilier permet une attribution au Munzingen B.

\section{St. 171}

Sépulture d'un adulte (individu 1) de sexe féminin (?) et d'un enfant (individu 2) dans une fosse circulaire. Les sujets reposent sur le même niveau, une quinzaine de centimètres au-dessus du fond du creusement. Il s'agit très probablement d'un dépôt simultané.

Position: sur le ventre, bras ramenés vers le haut du corps et membres inférieurs légèrement fléchis vers la gauche (individu 1); plaqué contre la paroi, sur le côté droit, membres inférieurs disjoints fléchis (individu 2).

Orientation: sud/nord (individu 1); nord-est/sud-ouest (individu 2).

Mobilier: tessons peu caractéristiques sur toute la hauteur du comblement.
Datation: Michelsberg III d'après une datation radiocarbone (GRA31505: $5135 \mathrm{BP} \pm 35$ ans, soit 39803810 av. J.-C. à $1 \sigma)$.

Particularité: enfoncement sur le crâne de l'enfant.

\section{ROSHEIM (LOTISSEMENT SAINTE-ODILE)}

Bibliographie: Jeunesse, 1992; Nickel, 1998.

\section{St. 419}

Inhumation d'un adulte de sexe féminin (?) dans une fosse circulaire (diam.: 1,85 m; prof.: $1 \mathrm{~m}$ ).

Position: sur le dos, membres inférieurs fléchis vers la droite; bras droit sur le pubis, bras gauche ramené sur la poitrine.

Orientation: sud-nord.

Mobilier: gros fragment de meule en grès contre les jambes ainsi que des tessons, des fragments de torchis, de grès et d'os animaux.

Datation: Michelsberg IV.

Particularité: enfoncement du crâne.

\section{ROSHEIM (ROSENMEER)}

Bibliographie: Rohmer, 1995.

\section{St. 19}

Inhumation de deux adultes de sexe féminin (individus 1 et 2), d'un enfant (individu 3) et d'un adulte de sexe indéterminé (individu 4) dans un complexe de fosses. Il s'agit d'une inhumation à étages, c'est-à-dire que les sujets ont été inhumés à des profondeurs différentes. Le premier (individu 4) a été déposé sur le fond de la fosse, le visage contre la paroi. L'une des femmes et l'enfant (individus 2 et 3 ) ont été retrouvés $0,50 \mathrm{~m}$ au-dessus du premier. Enfin, la seconde femme reposait 0,60 $\mathrm{m}$ au-dessus des précédents.

Position: le squelette de l'individu 1 semble fortement perturbé et, d'après la photographie du rapport, incomplet. D’après les fouilleurs, cet état est dû aux labours; les individus 2 et 3 ont été inhumés ensemble, le bassin de l'enfant reposant directement sur la cuisse gauche de l'adulte, les bustes serrés l'un contre l'autre et les visages tournés l'un vers l'autre; l'adulte (individu 2) reposait sur le dos, les membres inférieurs faiblement fléchis avec les genoux écartés et les pieds joints; quant à l'enfant (individu 3), il a été placé sur le dos, le buste légèrement tourné vers l'individu 2, les membres inférieurs légèrement fléchis; sur le dos, les bras alignés le long du corps, les membres inférieurs fortement fléchis avec les talons ramenés sous le bassin (individu 4). 
Orientation: ouest-sud-ouest/est-nord-est (?; individu 1); est-sud-est/ouest-nord-ouest (individus 2-4).

Mobilier: tessons Grossgartach et Michelsberg.

Datation: Michelsberg III/IV.

\section{ROSHEIM (SABLIÈRE MAETZ)}

Bibliographie: Thévenin et al., 1978.

Emplacement 7

Inhumation de deux adultes de sexe féminin (individu supérieur: 20/30 ans; individu inférieur: 35/55 ans) ; creusement non renseigné.

Position: l'individu supérieur (femme adulte 20 à 30 ans) est couché sur le côté droit, membres inférieurs repliés et bras en extension en avant du visage. L'individu inférieur (femme adulte 35 à 55 ans) est «allongé sur le ventre, face contre le sol avec les bras repliés et plaqués contre le thorax. Quant à la partie inférieure du corps, elle est en revanche placée sur le côté gauche avec les jambes pliées». Orientation: non renseignée.

Mobilier: un tesson au niveau du pied et plusieurs fragments de grès sur la poitrine de l'individu supérieur; un fragment de meule sous l'épaule droite de l'individu inférieur.

Datation: Néolithique récent.

\section{Emplacement 8}

Un squelette détruit; aucun autre renseignement.

Datation:?

\section{STRASBOURG-KOENIGSHOFFEN}

Bibliographie: Forrer, 1911; Gallia Préhistoire, 6, 1963, p. 182-183.

\section{Tombe 1902}

Deux adultes inhumés l'un contre l'autre (individu 1 au sud et 2 au nord); creusement non renseigné.

Position: tous deux allongés sur le dos, les bras alignés le long du corps et les membres inférieurs en extension. Orientation: nord-ouest/sud-est.

Mobilier: une perle en jais au niveau du poignet de l'individu 1, 2 vases à droite de la tête de l'individu 1, plusieurs tessons entre les deux individus au niveau de leurs épaules, d'autres tessons au niveau de la jambe gauche de l'individu 2, une armature de flèche en silex au niveau du thorax et 2 galets au niveau du bassin de l'individu 2; chaque défunt portait en outre un collier composé de perles en calcaire et en jais.

Datation: Bischheim.

Tombe 1

Tombe détruite.

Datation: ?

\section{Tombe 2}

Inhumation d'un adulte de sexe masculin; creusement non renseigné.

Position: allongé sur le dos, les bras alignés le long du corps et les membres inférieurs en extension.

Orientation: nord-ouest/sud-est.

Mobilier: un tesson à droite du genou droit, une valve de moule d'eau douce perforée à hauteur de la main gauche, un bracelet en os et un bracelet composé de perles calcaires portés au poignet droit, un collier composé de perles en calcaire, une armature de flèche en silex à droite du genou droit ainsi qu'une «motte de terre rougeâtre » à gauche du crâne.

Datation: Néolithique moyen.

Tombe 3

Tombe détruite.

Datation:?

Tombe 4

Tombe détruite.

Datation:?

\section{HAUT-RHIN}

\section{BOLLWILLER}

Bibliographie: Gallay, 1970; Nickel, 1997.

Inhumation de huit individus, dont cinq hommes, deux femmes et un enfant, découverte à 2,50 m de profondeur; creusement non renseigné.

Position: non renseignée.

Orientation: non renseignée.

Mobilier: tessons, fragments d'os animaux et deux squelettes de suidé.

Datation: Néolithique récent?

\section{Colmar (AÉrodrome)}

Site fouillé en 2008 (P. Lefranc, inédit) ayant révélé 75 creusements circulaires de type silo. Le secteur fouillé a livré 13 inhumations primaires réparties dans 10 structures - 9 inhumations simples et 4 inhumations dans un même creusement - ainsi que 11 dépôts secondaires. Le nombre total de creusements ayant livré des restes humains sur ce secteur est de 19 (un des dépôts secondaires, une calotte crânienne, a été découvert dans le remplissage, au-dessus d'une inhumation).

\section{St. 11}

Un fragment de pariétal droit adulte.

Mobilier: aucun.

Datation: Néolithique récent probable. 


\section{St. 12}

Un fragment de diaphyse fémorale droite adulte.

Mobilier: aucun.

Datation: Néolithique récent probable.

\section{St. 14}

Bloc craniofacial d'adulte en position secondaire.

Mobilier: un pendentif perforé sur défense de suidé déposé à $17 \mathrm{~cm}$ au nord du bloc craniofacial, et au même niveau que celui-ci.

Datation: Néolithique récent probable.

\section{St. 17}

Inhumation d'un adulte dans une fosse de plan circulaire (diam.: 1,74 m; prof.: 0,92 m). Ses restes sont fortement remaniés. Les déplacements observés résultent très probablement d'une réintervention postdécompositionnelle: certains indices, comme la position tout à fait particulière des clavicules, comme «rangées", parallèles l'une à l'autre, à l'est du bloc craniofacial, ou celle des deux côtes, également parallèles l'une à l'autre mais aussi perpendiculaires aux clavicules, ne laissent que peu de doute quant au caractère anthropique de cette réintervention.

Orientation: sud-est/nord-ouest.

Mobilier: à une dizaine de centimètres sous le niveau d'inhumation de l'individu remanié gisait le squelette complet d'un jeune sanglier décomposé en espace colmaté.

Datation: Néolithique récent (Gd-30169: $5310 \pm 170$ BP, 4330 (68.2\%) 3975 cal. B).

\section{St. 20}

Inhumation d'un adulte de sexe masculin dans une fosse circulaire (diam.: 1,30 m; prof.: 0,50 m).

Position: sur le côté gauche.

Orientation: nord-nord-ouest/sud-sud-est.

Mobilier: aucun.

Datation: Néolithique récent probable.

\section{St. 23A}

Inhumation d'un sujet dans une fosse circulaire (diam.: $1,30 \mathrm{~m}$ ) ; prof.: 0,68 m).

Position: procubitus, membres inférieurs en extension formant un angle d'environ $90^{\circ}$ avec le tronc et membres supérieurs repliés sous le thorax.

Orientation: nord-est/sud-ouest.

Mobilier: au même niveau et en contact strict avec le squelette, deux objets composés de 56 perles en cuivre probablement importées de Suisse occidentale (cuivre de Mondsee) ; à une vingtaine de centimètres au-dessus du crâne, le comblement recelait des fragments osseux appartenant à un gigot de chevreuil.
Datation: Munzingen B d'après la typologie des perles (Cortaillod tardif).

\section{St. 26}

Inhumation d'un adulte dans une fosse subcirculaire (long. : 1,60 m; larg. : 1,30 m; prof.: 0,60 m).

Position: sur le côté droit.

Orientation: nord-nord-ouest/sud-sud-est.

Mobilier: aucun.

Datation: Néolithique récent probable.

St. 33

Un fragment de calotte crânienne découvert parmi les os d'un faon.

Mobilier: aucun.

Datation: Néolithique récent probable.

\section{St. 41}

Inhumation d'un enfant ( $5 / 7$ ans) dans une fosse circulaire (diam. : 1,40 m; prof.: 0,88 m). Le corps repose à 0,74 $\mathrm{m}$ au-dessus du fond du creusement.

Position: sur le côté droit, en très léger décubitus, au centre de la fosse.

Orientation: ouest-sud-ouest/est-nord-est.

Mobilier: une jatte complète en position fonctionnelle.

Datation: Munzingen B.

\section{St. 45}

Une extrémité de côte adulte (tête, col et tubercule) et un quatrième métacarpien droit adulte.

Mobilier: aucun.

Datation: Néolithique récent probable.

St. 48

Une extrémité proximale d'ulna droit adulte et un cinquième métacarpien droit adulte.

Mobilier: aucun.

Datation: Néolithique récent probable.

St. 49

Inhumation de deux adultes et de deux enfants dans une fosse circulaire (diam. : 1,34 m; prof. : 0,96 m). Trois épisodes de dépôt, séparés par des couches de sédiments de 16 à $20 \mathrm{~cm}$ de puissance, ont été observés dans cette structure:

- premier dépôt (individus C et D) à $16 \mathrm{~cm}$ au-dessus du fond du creusement. Un adulte et un enfant en contact strict. Adulte en position fléchie sur le côté droit, orienté est-nord-est/ouest-sud-ouest; enfant entre 9 et 10 ans en position désordonnée;

- deuxième dépôt (individu B) à $38 \mathrm{~cm}$ au-dessus du fond du creusement et à $20 \mathrm{~cm}$ au-dessus du premier dépôt. Restes mal conservés d'un enfant entre 18 et 36 mois en décubitus, orienté nord-sud; 
- troisième dépôt (individu A) à $56 \mathrm{~cm}$ au-dessus du fond du creusement et à $16 \mathrm{~cm}$ au-dessus du deuxième dépôt. Femme de plus de 50 ans en décubitus, membres inférieurs fléchis, orientée est-nord-est/ouest-sud-ouest. Mobilier: aucun.

Datation: Néolithique récent.

\section{St. 51}

Inhumation d'un adulte dans une fosse circulaire (diam.: 1,38 m ; prof.: 1,10 m).

Position: inhumé en position fortement contractée sur le côté droit, à $15 \mathrm{~cm}$ au-dessus du fond du creusement. Orientation: est-sud-est/ouest-nord-ouest.

Autres restes humains: un fragment de calotte crânienne a été retrouvé au sein du remplissage, à $80 \mathrm{~cm}$ au-dessus de l'inhumation.

Datation: Néolithique récent probable.

St. 52

Inhumation d'un individu dans une fosse circulaire (diam.: 1,65 m; prof.: 0,78 m).

Position: en décubitus, membres inférieurs fléchis.

Orientation: ouest-sud-ouest/est-nord-est.

Mobilier: aucun.

Datation: Néolithique récent probable.

\section{St. 56}

Inhumation d'un adulte de sexe masculin (20/29 ans) dans une circulaire (diam. : 1,40 m; prof.: 0,60 m).

Position: sur le côté gauche, membres inférieurs fléchis. Orientation: nord-est/sud-ouest.

Mobilier: poinçon en os, déposé à $15 \mathrm{~cm}$ à l'est du visage ainsi que les restes d'un suidé partiellement remanié décomposé en espace non colmaté, situé à une douzaine de centimètres sous l'individu.

Datation: Néolithique récent probable.

St. 65

Une extrémité proximale de tibia droit adulte.

Mobilier: aucun.

Datation: Néolithique récent probable.

St. 66

Dépôt secondaire (ulna, coxal, scapula, mandibule, clavicule; $\mathrm{NMI}=1$ ) dans une fosse circulaire (diam.: 1,25 $\mathrm{m}$; prof.: 0,46 m). Les restes humains reposent sur un même niveau, à une vingtaine de centimètres au-dessus du fond du creusement.

Mobilier: aucun.

Datation: Néolithique récent probable.

\section{St. 67}

Un fragment de calcaneus droit adulte.

Mobilier: aucun.
Datation: Néolithique récent probable.

\section{St. 110}

Inhumation d'un enfant (6/7 ans) dans une fosse circulaire (diam.: 1,64 m; prof.: 0,64 m). Le corps s'est décomposé en espace vide; le bloc craniofacial a été prélevé et repositionné à $80 \mathrm{~cm}$ du squelette.

Position: sur le côté gauche, en position contractée.

Orientation: ouest-nord-ouest/est-sud-est.

Mobilier: aucun.

Datation: Néolithique récent probable.

\section{DIDENHEIM (LERCHENBERG)}

Bibliographie: Lambach, 1986; Schweitzer, 1987.

Fosse 2

Inhumation d'un immature de sexe masculin (13/15 ans) dans une fosse circulaire (diam.: 1,80 m; prof.: 2,70 m).

Position: «decubitus dorsal, légèrement tourné vers la droite» (Schweitzer, 1987) ; membres inférieurs légèrement fléchis.

Orientation: nord/sud.

Mobilier: bouteille et grande jarre «dont les fragments ont été retrouvés au-dessus et juste en dessous du squelette», poinçon, lame en silex, tessons et fragments de faune (Schweitzer, 1987).

Datation: Munzingen A d'après le mobilier, plus tardif d'après une datation radiocarbone (Ly-2230: $4930 \mathrm{BP} \pm 30$ ans, soit 3750-3655 av. J.-C. à 1 o).

\section{Fosse 5}

Inhumation de trois enfants ( 2 ans, $4 / 5$ ans et $7 / 8$ ans) reposant au fond d'une fosse circulaire (diam.: 1,50 m; prof.: 1,20 m). Il s'agit d'un dépôt simultané.

Position: deux individus fléchis sur le côté gauche, le troisième sur le côté droit.

Orientation: nord-sud.

Mobilier: une jarre dont les fragments reposaient au niveau des crânes.

Datation: Munzingen A.

\section{Fosse 13}

Inhumation d'un adulte, d'un immature (12/15 ans) et d'un enfant (6/7 ans) dans une fosse circulaire (diam.: 1,50 m; prof.: 1,20 m). Il s'agit d'un dépôt simultané.

Position: deux individus (adulte et adolescent) couchés sur le côté gauche, membres inférieurs fléchis, encadrant l'enfant (position non renseignée: d'après les croquis publiés par Schweitzer, l'enfant paraît couché sur le côté droit, membres inférieurs fléchis).

Orientation: non renseignée. 
Mobilier: un broyon.

Datation: Munzingen A ?

Particularité: enfoncement sur le crâne d'un des enfants.

\section{DIDENHEIM (ZAC DES COLLINES)}

Bibliographie: inédit (fouille Antea-Archéologie); étude en cours.

St. 638

Inhumation de trois jeunes enfants ( $2 / 4$ ans, $3 / 5$ ans) dans une fosse ovale irrégulière (long.: 2,20 m; larg.: 1,20 m; prof. : 0,15 m). Les restes d'un des enfants ont été placés à peu près au centre de la fosse, ceux des deux autres à une trentaine de centimètres plus au sud. Il est possible que le premier ait été inhumé entier, mais une partie de son squelette a été détruite lors de la fouille, si bien que l'on ne peut pas en être assuré. Seuls son crâne et les os de ses deux bras ont été retrouvés. Il a peut-être été placé sur le ventre. Si on considère qu'il a été inhumé entier, il devait être orienté nord-ouest/ sud-est. La localisation du crâne entre les bras indique éventuellement que ce corps a été manipulé, mais là encore sans certitude. La situation est plus claire avec les deux autres enfants dont seuls les crânes ont été retrouvés. Sous ces crânes, se trouvait également un os long humain. Il est difficile de dire à quel individu il appartenait.

Position: sur le ventre?

Orientation: nord-ouest/sud-est.

Mobilier: aucun.

Datation: Néolithique récent?

\section{DIDENHEIM/MORSCHWILLER-LE-BAS} (ROCADE OUEST)

Bibliographie: Zehner et al., 2002; Denaire, 2007.

\section{Zone 1/St. 1}

Inhumation d'un adulte et d'un enfant en bas âge dans une fosse circulaire (diam.: $2 \mathrm{~m}$; prof.: 0,90 m). L'enfant a été retrouvé à une dizaine de centimètres sous l'adulte.

Position: sur le dos, bras repliés sur le thorax et membres inférieurs fléchis vers la gauche (adulte); le squelette de l'enfant est mal conservé.

Orientation: nord-sud (adulte) et nord-est/sud-ouest (enfant).

Mobilier: deux vases incomplets.

Datation: Munzingen A d'après une datation radiocarbone (Ly-2233: $4930 \mathrm{BP} \pm 30$ ans, soit 3940-3770 av. J.-C. à $1 \sigma$ ), Munzingen $\mathrm{B}$ d'après le mobilier.
Zone 2/St. 8

Inhumation de trois enfants et d'un adulte dans une fosse circulaire (diam. : 1,20 m; prof. : 0,40 m). Les corps de deux des enfants (individus 2 et 3 ) ont été placés sur le fond de la fosse. Celui de l'individu 2 est complet mais présente plusieurs perturbations et déconnexions. Le crâne de l'individu 3, probablement prélevé, est manquant. Une dizaine de centimètres au-dessus de ces deux premiers individus reposaient les restes incomplets et dispersés des deux autres défunts (individu la et b: crânes, os longs, quelques côtes et fragments de mains et de pieds. Un crâne en connexion avec les cervicales). Il peut naturellement s'agir de dépôts secondaires, mais rien ne permet d'exclure l'éventualité de manipulations in situ.

Position: allongé sur le côté droit, en position désordonnée (individu 2) ; sur le côté gauche en position fléchie, avant-bras repliés sur le thorax (individu 3).

Orientation: nord-sud (individu 2); sud-est/nord-ouest (individu 3).

Mobilier: un collier composé de 2 craches de cerf et d'un galet perforés (individu 3).

Datation: Munzingen $\mathrm{B}$ d'après une datation radiocarbone (Ly-2234: 4925 BP \pm 35 ans, soit 3760-3650 av. J.-C. à $1 \sigma)$.

\section{Zone 3/St. 28}

Inhumation de deux adultes et de deux enfants dans une fosse subcirculaire (long.: 1,25 m; larg.: 1,30 m; prof.: 1,15 m). Trois des défunts ont été placés les uns sur les autres: le corps du premier inhumé (individu 4) gisait non loin du fond de la fosse, contre la paroi de la fosse dont il épousait le tracé. Le second adulte (individu 3) a été déposé directement sur le précédent, lui-même étant recouvert par le corps d'un des enfants (individu 2). Les ossements perturbés (décomposition dans un contenant souple ?) du second enfant (individu 1) gisaient à quelques centimètres du premier dépôt, au même niveau.

Position: sur le dos (individu 1; la position des membres n'est pas connue); sur le côté droit, les avant-bras repliés, le membre inférieur gauche en extension, le gauche légèrement fléchi (individu 2); sur le côté gauche, les avant-bras repliés avec les mains devant le visage, les membres inférieurs fortement fléchis avec les talons ramenés sous le bassin (individu 3); sur le dos, membres inférieurs en extension (individu 4).

Orientation: nord-est/sud-ouest (individus 1 et 2); sudnord (individu 3); nord-sud (individu 4). 
Mobilier: dépôt funéraire composé d'une écuelle à fond plat et d'un gobelet en bois de cerf placés à quelques centimètres en avant du visage du défunt 3 .

Datation: Munzingen A d'après le gobelet en bois de cerf (daté en Suisse des XXXIX ${ }^{\mathrm{e}}$ et XXXVIII ${ }^{\mathrm{e}}$ s. av. J.-C.), plus tardif d'après les résultats de deux datations ${ }^{14} \mathrm{C}$ (Ly2235: $4940 \mathrm{BP} \pm 30$ ans et Ly-2236: $4885 \mathrm{BP} \pm 40$ ans, dont le cumul des probabilités donne la fourchette 3710 3640 av. J.-C. à 1 o).

\section{ESCHENTZWILLER (BRANDSTÄTTE)}

Bibliographie: Wolf, 1979

Inhumation d'un individu dans une fosse ovale (dim.: $1,90 \mathrm{~m} \times 1,50 \mathrm{~m})$.

Position: individu couché sur le côté droit, membres inférieurs fléchis et bras repliés.

Orientation: sud-nord.

Mobilier: un vase complet à deux anses.

Datation: Proto-Munzingen.

\section{KATZENTHAL (DORFBURG)}

Bibliographie: Eich-Francke, 1967.

Inhumation de trois enfants dans une fosse circulaire (diam. : 1,10 m; prof. : $1 \mathrm{~m}$ ).

Position: sur le côté droit, les avant-bras fléchis avec les mains ramenées devant le visage et les membres inférieurs fortement fléchis.

Orientation: non renseignée.

Mobilier: petits vases entiers et tessons.

Datation: Néolithique récent?

\section{MERXHEIM (TRUMMELMATTEN)}

Bibliographie: Treffort, Dumont, 2000.

Fosse 57

Le crâne, la mandibule et des fragments des deux premières vertèbres cervicales d'un enfant (6/11 ans) dans une fosse circulaire (diam. : 1,40 m; prof.: 0,85 m).

Mobilier: 3 perles discoïdales en roche noire, 4 perles discoïdales en calcaire et 4 perles en forme de hache en roche blanche.

Datation: du Bischheim au début du Munzingen.

\section{RIEDISHEIM (BEAU-SITE)}

Bibliographie: Schweitzer, Fulleringer, 1973.

Fosse 1

Inhumation d'un enfant (4/5 ans) dans une fosse circulaire (diam.: 0,90 m; prof.: 1,50 m).
Position: non renseignée (membres inférieurs retrouvés sur les déblais).

Orientation: non renseignée.

Mobilier: tessons, fragments de torchis et d'os animaux issus du comblement

Datation: Munzingen A.

\section{RIEDISHEIM (RUE DES VIOLETTES)}

Bibliographie: Schweitzer, Fulleringer, 1973.

\section{Fosse 1}

Inhumation d'un adulte et de deux enfants (dont l'un âgé de 7 ans environ) dans une fosse circulaire (diam.: 1,60 m; prof. : 2,50 m). L'adulte (individu 1) et l'un des enfants (individu 2, incomplet ?) reposaient sur le fond de la fosse, tandis que les restes du second enfant (individu 3) ont été retrouvés $0,70 \mathrm{~m}$ plus haut, séparés des deux autres corps par une couche stérile. Le fond de la fosse est rubéfié et couvert d'une couche cendreuse. L'individu du niveau supérieur est englobé dans une couche recelant des cendres, du charbon de bois et des fragments de pisé.

Position: sur le dos, les membres inférieurs repliés (individu 1); non renseignée (individu 2); «fortement replié» (individu 3) (Schweitzer, Fulleringer, 1973).

Orientation: non renseignée.

Mobilier: tessons, fragments de hache, de torchis, d'os animaux et de bois de cerf.

Datation: Munzingen.

Particularité: enfoncement sur le crâne de l'adulte.

\section{SOULTZ (BUHLFELD)}

Bibliographie: Chatton, 1974 et 1976; Bonnet, PlouinMantzer, 1979.

Deux inhumations fouillées en 1971 par Chatton et une inhumation quadruple fouillée en 1975 par R. et J. Schweitzer.

Fosse 1971/1

Inhumation d'un adulte de sexe indéterminé (40/70 ans) dans une fosse circulaire (diam.: 0,80 m; prof.: $1,80 \mathrm{~m}$ ).

Position: «sur le côté, bras et membres inférieurs repliés et ramenés sur la poitrine» (Chatton, 1976).

Orientation: est/ouest.

Mobilier: quelques tessons et un fragment d'os animal. Datation: Néolithique récent?

Fosse 1971/2

Inhumation d'un adulte de sexe masculin (25/40 ans) 
dans une fosse circulaire (diam.: 0,80 m; prof. : 1,80 m). Position: sur le dos, «jambes écartées repliées sur la poitrine»(Chatton, 1976).

Orientation: ouest/est.

Mobilier: un squelette de jeune lièvre reposait sur la poitrine de l'individu.

Datation: Néolithique récent?

\section{Fosse 1}

Inhumation d'un adulte de sexe masculin (40/60 ans; individu 1), de deux enfants (7/8 ans et 2 ans; individus 2 et 3) et d'un nouveau-né (individu 4) dans une fosse probablement circulaire (prof. : 1,40 m). D'après les clichés de fouille, l'adulte semble placé au centre de la fosse, tandis que l'un des enfants (individu 2) a été plaqué contre la paroi de la fosse, en face de l'adulte, les pieds "accolés à la face latérale gauche du crâne de l'adulte». Contrairement à ce qui a été décrit, l'enfant semble plus bas que l'adulte. Le corps du second enfant n'apparaît pas sur les photographies. Il aurait été retrouvé au niveau de l'enfant décrit. Les deux fragments de crâne du nouveau-né ont curieusement été retrouvés au lavage dans le crâne de l'individu 2.

Position: sur le côté droit, membres inférieurs fléchis (individu 1) ; sur le côté gauche, les membres inférieurs en extension (individu 2); non renseignée (individu 3 et 4).

Orientation: ouest/est (individu 1); nord-ouest/sud-est (individu 2).

Mobilier: aucun.

Datation: Néolithique récent?

\section{UNGERSHWEIM (DESSERTE ROUTIÈRE DU BIOSCOPE)}

Bibliographie: Lefranc et al., 2009.

Petite nécropole de la transition $\mathrm{V}^{\mathrm{e}}-\mathrm{IV}^{\mathrm{e}}$ millénaire rassemblant dix-sept individus, tous orientés sud-est/ nord-ouest et inhumés sur le dos, membres inférieurs en extension. Datation: BORS récent ou Proto-Munzingen.

\section{Sép. 1}

Inhumation d'un adulte; creusement non renseigné.

Position: allongé sur le dos.

Orientation: sud-est/nord-ouest.

Mobilier: aucun.

Datation: BORS récent ou Proto-Munzingen?

\section{Sép. 2}

Inhumation très mal conservée; creusement non renseigné.

Position: non renseignée.

Orientation: sud-est/nord-ouest.
Mobilier: une petite ébauche de hache en pélite quartz et un ciseau en schiste noduleux, déposés à proximité du crâne.

Datation: BORS récent ou Proto-Munzingen?

Sép. 1001

Inhumation de deux jeunes adultes; creusement non renseigné.

Position: sur le dos, bras alignés le long du corps et membres inférieurs en extension.

Orientation: sud-est/nord-ouest.

Mobilier: lame de hache polie en pélite-quartz de forme trapézoïdale élancée, à talon arrondi et tranchant convexe symétrique.

Datation: BORS récent ou Proto-Munzingen d'après une date radiocarbone (Poz-22532: 5190BP \pm 35 ans, soit 4039-3966 av. J.-C. à 1 o).

Sép. 1002

Quelques ossements sans ordre anatomique; aucun autre renseignement.

Sép. 1003

Inhumation d'un adulte; creusement non renseigné.

Position: sur le dos, bras alignés le long du corps et membres inférieurs en extension.

Orientation: sud-est/nord-ouest.

Mobilier: un grand fragment d'écuelle en calotte déposé aux pieds de l'individu.

Datation: BORS récent ou Proto-Munzingen?

Sép. 1004

Quelques ossements appartenant à un individu; creusement non renseigné.

Position: sur le dos, membres inférieurs en extension et orienté sud-est/nord-ouest, tête au sud-est.

Orientation: sud-est/nord-ouest.

Mobilier: aucun.

Datation: BORS récent ou Proto-Munzingen?

Sép. 1005

Inhumation mal conservée; creusement non renseigné.

Position: membres inférieurs en extension.

Orientation: sud-est/nord-ouest.

Mobilier: aucun.

Datation: BORS récent ou Proto-Munzingen?

Sép. 1006

Inhumation d'un adulte; creusement non renseigné.

Position: sur le dos, bras le long du corps, membres inférieurs en extension. Rotation du crâne imputable à une manipulation postdécompositionnelle.

Orientation: sud-est/nord-ouest.

Mobilier: aucun. 
Datation: BORS récent ou Proto-Munzingen?

Sép. 1007

Inhumation d'un adulte; creusement non renseigné.

Position: sur le dos, bras le long du corps et membres inférieurs en extension.

Orientation: sud-est/nord-ouest.

Mobilier: aucun.

Datation: BORS récent ou Proto-Munzingen?

Sép. 1008

Inhumation d'un adulte; creusement non renseigné.

Position: sur le dos, bras le long du corps et membres inférieurs en extension.

Orientation: sud-est/nord-ouest.

Mobilier: une lame de hache polie en pélite-quartz de forme trapézoïdale élancée, à talon pointu et tranchant convexe symétrique. L'objet reposait au contact des fragments du crâne de l'individu.

Datation: BORS récent ou Proto-Munzingen d'après une date radiocarbone (Poz-22533: 5180BP \pm 35 ans, soit 4038-3962 av. J.-C. à 1 б).

Sép. 1009

Ossements sans ordre anatomique; aucun autre renseignement.

Sép. 1010

Eléments du crâne et d'os longs sans ordre anatomique ; aucun autre renseignement.

Sép. 1011

Diaphyse isolée; aucun autre renseignement.

Sép. 1012

Inhumation d'un immature ; creusement non renseigné.

Position: sur le dos, bras le long du corps et membres inférieurs en extension.

Orientation: sud-est/nord-ouest.

Mobilier: aucun.

Datation: BORS récent ou Proto-Munzingen?

Sép. 1015

Inhumation d'un individu; creusement non renseigné.

Position: sur le dos, avec membres inférieurs en extension.

Orientation: sud-est/nord-ouest.

Mobilier: aucun.

Datation: BORS récent ou Proto-Munzingen?

Sép. 1016

Inhumation d'un adulte; creusement non renseigné.

Position: sur le dos, bras le long du corps et membres inférieurs en extension; fracture du bras gauche.

Orientation: sud-est/nord-ouest.

Mobilier: aucun.
Datation: BORS récent ou Proto-Munzingen d'après une date radiocarbone (Poz-22564: $519 \mathrm{BP} \pm 30$ ans, soit 4038-3970 av. J.-C. à 1 o).

\section{Sép. 1017}

Inhumation d'un individu; creusement non renseigné. Position: sur le dos, membres inférieurs en extension.

Orientation: sud-est/nord-ouest.

Mobilier: aucun.

Datation: BORS récent ou Proto-Munzingen?

\section{WETTOLSHEIM (KOENIGSBREITE)}

Bibliographie: Gallay, 1970.

Une sépulture double; creusement non renseigné.

Position: au moins un individu en position contractée.

Orientation: non renseignée.

Mobilier: tessons, cendres, faune et torchis.

Datation: Néolithique récent.

\section{SUD DU BADE}

\section{ISTEIN (ISTEINER KLOTZ)}

Bibliographie: Badische Fundberberichte, 2, 1929-1932; Nikkel, 1998.

Lors de la fouille de cette grotte, un coxal d'enfant (9-10 ans) a été découvert dans le comblement d'une fosse néolithique (hache, céramique, os animaux) entaillant une couche paléolithique. Une mandibule d'enfant a également été découverte dans une couche attribuée au Néolithique.

Datation: la datation de ces restes humains pose problème compte tenu des risques de mélanges avec des éléments plus récents.

\section{JECHTINGEN (HUMBERG)}

Bibliographie: Badische Fundberberichte, 18, 1948-1950; Nickel, 1998.

Inhumation d'un adulte de sexe masculin dans une fosse de forme indéterminée. À quelques centimètres sous les pieds, concentration de fragments d'ossements appartenant à un humain et à un suidé.

Position: sur le dos, membres inférieurs repliés (Rückenhocker).

Orientation: est-ouest.

Mobilier: aucun, hormis le squelette de suidé.

Datation: Néolithique récent?

\section{KIECHLINSBERGEN (EDELBERG)}

Bibliographie: Nickel, 1998. 


\section{Squelette 1}

Inhumation d'un adulte sans crâne dans une fosse de forme non renseignée.

Position: non renseignée.

Orientation: non renseignée.

Mobilier: aucun.

Datation: Néolithique récent?

Squelette 2

Inhumation d'un adulte dans une fosse de forme non renseignée.

Position: sur le côté, les membres inférieurs fléchis.

Orientation: non renseignée.

Mobilier: tessons.

Datation: Munzingen.

\section{KLEINKEMS (KACHELFLUH-HÖHLE)}

Bibliographie: Nickel, 1998.

Nombreux fragments appartenant à trois individus en déconnexion (un homme, une femme et un indéterminé) découverts dans une petite niche aménagée contre une paroi de la cavité (1,40 m x 0,90 m; prof.: $0,60 \mathrm{~m})$. Les os longs des bras et des jambes, ainsi qu'un crâne, étaient plaqués contre la paroi de la niche. Le remplissage de la fosse a livré d'autres ossements humains, de la faune, de la céramique attribuée au Néolithique récent ainsi qu'un gobelet en bois de cerf. Datation: Munzingen A et B.

\section{LEISELHEIM (STRASSENKREUZUNG LEISELHEIM- KÖNIGSCHAFFHAUSEN-SASBACH)}

Bibliographie: Kimmig, 1950.

Inhumation détruite; creusement non renseigné.

Position: non renseignée.

Orientation: est-nord-est/ouest-sud-ouest.

Mobilier: au moins un vase décoré.

Datation: BORS.

\section{MUNZINGEN (MUNZINGEN BERG ET LINDENBERG)}

Bibliographie: Kimmig, 1941-1947; Schmid, 1958; Lüning, 1968; Nickel, 1998.

Fossé

Mâchoire et dent isolées.

Mobilier (à proximité des restes humains) : non renseignée. Datation: Munzingen probable.

\section{St. 25}

Fragments de fémur dans une fosse de forme non renseignée. Mobilier: fragments de meule, de roche et d'os animaux. Datation: Munzingen probable.

\section{St. 49}

Un fragment de bassin dans une fosse circulaire (diam.: $1,30 \mathrm{~m}$; prof. : $0,95 \mathrm{~m}$ ).

Mobilier: tessons, lame de silex, fragments de meule, de roche, d'os animaux et de bois de cerf.

Datation: Munzingen.

Fosse Knobel II/3

(Doppelbeutelgrube): inhumation d'un adulte dans le creusement ouest d'une fosse-silo double (prof. : 2,70 m).

Position: désordonnée.

Orientation: non renseignée.

Mobilier: tessons.

Datation: Munzingen B.

\section{NIEDER-SCHWÖRSTADT («RAMENKOSTETS»)}

Bibliographie: Badische Fundberberichte, 22, 1962; Nickel, 1998.

\section{Bestattung I}

Inhumation d'un enfant en grotte.

Position: étendu sur le dos, membres inférieurs en extension. Orientation: nord-sud.

Datation: ?

\section{Bestattung II}

Inhumation d'un enfant en grotte.

Position: sur le côté.

Orientation: nord-sud.

Datation: ?

Remarque: la datation de ces deux inhumations pose problème. Elle ne repose que sur la présence d'une occupation du Néolithique récent attestée par la découverte d'un vase en 1963. Précisons que ces deux inhumations recoupent un niveau mésolithique.

\section{SASBACH (WÖRTHSTÜCK)}

Bibliographie: Fundberichte aus Baden-Württemberg, 10, 1985, p. 474-478 et Abb. 10-14.

Grab 1

Inhumation d'un adulte dans une fosse rectangulaire ajustée à son contenu (dim.: 2,10 m x 0,85 m).

Position: allongé sur le dos, membres inférieurs en extension. Orientation: nord-ouest/sud-est.

Mobilier: une lame de hache perforée au niveau de l'épaule droite, une lame d'herminette le long de l'avant-bras gauche et une jatte décorée écrasée à gauche du crâne. Datation: Bruebach-Oberbergen.

\section{WEISWEIL (OBER ENDINGER WEG)}

Bibliographie: Stöckl et Neubauer-Saurer, 1990. 


\section{Fo[sse ?] 8}

Inhumation d'un adulte dans une fosse subcirculaire (diam.: 1,40 m; prof.: ?).

Position: sur le dos, les membres inférieurs légèrement pliés et écartés. Le crâne n'est plus en connexion avec les cervicales, mais repose sur le thorax.

Orientation: sud-ouest/nord-est.

Mobilier: tessons, galet, fragments d'os animaux, de bois de cerf et de valve de moule d'eau douce.

Datation: Bischheim rhénan d'après le tesson. Toutefois, le lien entre le tesson et l'inhumation n'est pas certain.

\section{WOLFENWEILER-LEUTERSBERG \\ (IN DEN KRAUTLÄNDERN)}

Bibliographie: Badische Fundberberichte, 15, 1939; 21, 1958; Nickel, 1998.
Inhumation d'un individu; creusement non renseigné.

Position: allongé sur le dos.

Orientation: est-ouest.

Mobilier: fragments d'un vase dispersé au niveau du corps.

Datation: Munzingen.

\section{REMERCIEMENTS}

Nos vifs remerciements vont aux anthropologues de terrain avec qui nous collaborons quotidiennement et dont les travaux ont nourri cette étude: E. Boës (Inrap), Émilie Cartier et Amandine Mauduit (Antea-Archéologie). Toute notre gratitude va également aux responsables d'opérations qui nous ont ouvert leurs archives, souvent riches de données inédites: Y. Henigfeld, M. Châtelet, Y. Thomas et F. Schneikert (Inrap), C. Toullec (AnteaArchéologie). 


\title{
BIBLIOGRAPHIE
}

\author{
ABRÉVIATIONS \\ APRAA Association pour la promotion de la recherche archéologique en Alsace. \\ DFS Document final de synthèse. \\ SPF Société préhistorique française. \\ SRA Service régional d'archéologie.
}

\section{ARBOGAST R.-M.}

1989: «Le village Michelsberg des Hautes Chanvières à Mairy (Ardennes) -IV- Les animaux domestiques des fosses-silos», Gallia Préhistoire, 31, 1989, p. 139-158.

1998: «Contribution de l'archéozoologie du site Michelsberg de Mairy (Ardennes) à l'étude de la variabilité des faunes du Néolithique récent du nord de la France", in Die Michelsberger Kultur und ihre Randgebiete: Probleme der Entstehung, Chronologie und des Siedlungswesens, Kolloquium Hemmenhofen, 21-23 févr. 1997, Stuttgart Theiss, Materialhefte zur Archäologie in Baden-Württemberg, 43, p. 135-142.

\section{BAHN B.-W.}

1989: «Eine Grube der Baalberger Kultur mit Kultischem Befund von Melchendorf, Kr. Erfurt-Stadt», in SCHLETTE F. KaUfMANN D. (DIR.), Religion und Kult in Ur- und frühgeschichtlicher Zeit, Berlin, Akademie-Verlag, p. 165-170.

\section{BEECHING A.}

2003: «Organisation spatiale et symbolique du rituel funéraire chasséen en moyenne vallée du Rhône: première approche", in Chambon P., LeclerC J. (DIR.), Les Pratiques funéraires néolithiques avant 3500 av. J.-C. en France et dans les régions limitrophes, Paris, SPF (coll. Mémoires de la SPF, 33), p. 231-239.

Beeching A., CRUbézy E.

1998: «Les sépultures chasséennes de la vallée du Rhône», in GUILAINE J. (DIR.) Sépultures d'Occident et genèses des mégalithismes, Paris, éd. Errance, p. 147-164

\section{BEHRENDS R.-H.}

1988: «Zwei neue Erdwerke der Michelsberger Kutur in Bruchsal, Landkreis Karlsruhe», Archäologische Nachrichten aus Baden, 40-41, p. 3-8.

1998: «Neue Forschungen zur Michelsberger
Kultur im Kraichgau », in Die Michelsberger Kultur und ihre Randgebiete: Probleme der Entstehung, Chronologie und des Siedlungswesens, Kolloquium Hemmenhofen, 21-23 fév. 1997, Stuttgart, Komissionverlag K. Theiss, p. 115-119

BEYER A.-I.

1970: «Die Tierknochenfunde» in KOCH R. (DIR.), Das Erdwerk der Michelsbergerkultur auf dem Hetzenberg bei HeilbronnNeckargartach (coll. Forschungen und Berichte zur Vor- und Frühgeschichte in Baden-Würtemberg, 3-II), p. 7-43.

\section{BEYNEIX A.}

2003: Traditions funéraires néolithiques en France méridionale, Paris, éd. Errance, 287 p.

BIEL J.

1990: «Neue Untersuchungen in dem Michelsberger Erdwerk auf dem Hetzenberg von NeckarsulmObereisesheim, Kreis Heilbronn", Archäologische Ausgrabungen in BadenWürtemberg, p. 39-41.

BILloIN D., BAUdOUX J., BoËS E.,

Châtelet M., Colecchia A.,

HENIGFELD Y., LEFRANC P.

2005: Geispolsheim «Forlen», DFS de la fouille de sauvetage, Strasbourg, SRA Alsace, 99 p. et annexes.

\section{BLAIZOT F.}

2001: «Premières données sur le traitement des corps humains à la transition du Néolithique récent et du Néolithique final dans le Bas-Rhin», Gallia Préhistoire, 43 , p. $175-235$

Blouet V., Guillaume C.

1984: "Le Michelsberg en Lorraine", Revue archéologique de Picardie, 1, p. 125-145.

Blouet V., Guillaume C., Leesch D. 1980: «La grotte sépulcrale Michelsberg du
"Rudemont" à Arnaville (Meurthe-etMoselle) », in Le Néolithique de l'Est de la France, Sens, Société archéologique de Sens (coll. Cahier, 1), p. 135-143.

BOËS E.

2007: «Traitement des corps et violence durant le Néolithique récent en Alsace», Cahiers alsaciens d'archéologie d'art et d'histoire, 50, p. 3-10.

Böнм K.

1981: «Siedlungen und Gräber der Munchshöfener Kultur im Pfettrachtal, Landkreis Landshut, Niederbayern", Archäologische Jahr im Bayern, p. 68-69.

Böhm K., Pielmeier R.

1993: «Der älteste Mettalfund Altbayerns in einem Doppelgrab der Münchshöfener Gruppe aus Straubing», Archäologische Jahr im Bayern, p. 40-42.

BöHM K., SCHMOTZ K.

1991: Bestattungen der jungsteinzeitlichen Münchshöfener Gruppe, Deggendorf, Landratsamt (Archäologische Denkmäler im Landkreis Deggendorf, 5), $32 \mathrm{p}$.

BONNET A.

1899: Veröffentlichungen der Grossherzoglich Badischen, Sammlungen für Altertumsund Völkerkunde in Karlsruhe, 2, 39 p.

Bonnet C., Plouin-Mantzer S.

1979: «Compléments à la carte archéologique du Haut-Rhin (région colmarienne) -3- Les nouveaux sites pré- et protohistoriques", Cahiers alsaciens d'archéologie, d'art et d'histoire, 31, p. 5-21.

boujot C., CRubézy e., Duday H.

1991: «L'identité du Chasséen à travers les structures et pratiques funéraires ", in BEECHING A., BINDER D., Blanchet J.-C., Constantin C., 
DUBOUlOZ J., MARTINEZ R., MORDANT D., THÉVENOT J.-P., VAQUER J. (DIR.), Identité du Chasséen, Actes du colloque international de Nemours, 17-19 mai 1989, Nemours, APRAIF (coll. Mémoires du musée de Préhistoire d'Île-de-France, 4), p. 413-420.

\section{BOULESTIN B.}

2008: «Pourquoi mourir ensemble ? À propos des tombes multiples dans le Néolithique français », Bulletin de la SPF, 105, 1, p. 103-130.

\section{BRUZEK J.}

2002: «A Method for Visual Determination of Sex: Using the Human Hip Bone», American Journal of Physical Anthropology, 117 , p. $157-168$.

\section{CAPItAni A. DE}

2007: «La céramique du site d'Egolzwil 3 (marais de Wauwil, canton de Lucerne, Suisse)», in BESSE M. (DIR.), Sociétés néolithiques: des faits archéologiques aux fonctionnements socio-économiques, Actes du $27^{e}$ colloque interrégional sur le Néolithique, Neuchâtel, 1-2 oct. 2005, Lausanne, Société vaudoise d'histoire et d'archéologie (coll. Cahiers d'archéologie romande, 108), p. 207-213.

\section{CHARPENTIER G.}

1989: «Étude anthropologique des restes humains: fouilles des fossés néolithiques à Maizy (Aisne)", Revue archéologique de Picardie, 1-2, p. 109-110.

\section{Châtelet M.}

2002: Marlenheim La Peupleraie 2: Sur les marges d'une villa romaine et d'un habitat du haut Moyen Âge, DFS de fouille de sauvetage, Strasbourg, SRA Alsace, 2 vol., 125 p., 21 photos et annexes.

\section{CHÂTELET M. (DIR.)}

2009: Marlenheim Hofstatt (Bas-Rhin): des inhumations en silo néolithiques au quartier artisanal carolingien, DFS de fouille de sauvetage, Strasbourg, SRA Alsace, 3 vol.

\section{ChatTon M.}

1974: "Une nécropole néolithique près de Soultz", Annuaire de la société d'histoire des régions de Thann-Guebwiller, p. 159-161.

1976: "Nouvelles découvertes autour d'une nécropole néolithique près de Soultz", Annuaire de la société d'histoire des régions de Thann-Guebwiller, p. 72-75.

Coblenz W., Fritsche C.

1973: «Neolithische Siedlungsbestattung mit drei Skeletten und Resten weiterer Schädel aus Zauschwitz, Kr. Borna», Ausgabungen und Funde, 18, 6, p. 276-290.

Colas C., Manolakakis L., Thévenet C., BAIllieu M., BonNARdin S., DUBOUloz J., FARRUGIA J.-P., MAIGROT Y., NAZE Y., ROBERT B.

2007: «Le monument funéraire Michelsberg ancien de Beaurieux "la Plaine" (Aisne, France)", in BESSE M. (DIR.), Sociétés néolithiques: des faits archéologiques aux fonctionnements socio-économiques, Actes du $27^{7}$ colloque interrégional sur le Néolithique, Neuchâtel, 1-2 oct. 2005, Lausanne, Société vaudoise d'histoire et d'archéologie (coll. Cahiers d'archéologie romande, 108), p. 329-334

Croutsch C., ARbogast R.-M., LEPROVOST C., BOUQUIN D., SCHNEIDERSCHWIEN N., LANDOLT M.

2008: "Les sites d'Entzheim "In der Klamm" et de Geispolsheim "Schwobenfeld" (BasRhin): les occupations du Néolithique moyen et récent", Internéo, 7, p. 87-102.

\section{Croutsch C., Leprovost C.,}

BOUQUiN D., ARbogaST R.-M.,

PUTelat O., ENGEl E., Gerbasi F.

2007: Entzheim-Geispolsheim (Alsace, Bas-Rhin), Aéroparc (Lidl-CUS) -2- Les occupations néolithiques, DFS, SRA Alsace, 298 p.

\section{Crubézy E.}

1991: «Les pratiques funéraires du Chasséen dans la moyenne vallée du Rhône", in BEECHING A., BINDER D., Blanchet J.-C., CONSTANTIN C., DUBOULOZ J., MARTINEZ R., MORDANT D., THÉVENOT J.-P., VAQUER J. (DIR.), Identité du Chasséen, Actes du colloque international de Nemours, 17-19 mai 1989, Nemours, APRAIF (coll. Mémoires du musée de Préhistoire d'Île-de-France, 4), p. 393-398.

\section{DAllmeier L.-M., Froschauer W.}

1995: «Neue Befunde der Münshöffener Gruppe», Das Archäologsiche Jahr in Bayern, p. 32-34

Debut A., MASSET C.

1991: «Restes humains épars en milieu chasséen septentrional: recherches en cours", in BEECHING A., BINDER D., Blanchet J.-G., CONSTANTIN C., DUBOULOZ J., MARTINEZ R., MORDANT D., THÉVENOT J.-P., VAQUERJ. (DIR.), Identité du Chasséen, Actes du colloque international de Nemours, 17-19 mai 1989, Nemours, APRAIF (coll. Mémoires du musée de Préhistoire d'Île-de-France, 4), p. 409-412.

DEHN R., DIEGKMANN B.

1985: «Notice Sasbach, Wörthstück», Fundberichte aus Baden-Württemberg, 10, p. $474-478$.

\section{Delattre V., Bulard A., Gouge P.,}

\section{PIHUIT P.}

2000: "De la relégation sociale à l'hypothèse des offrandes: l'exemple des dépôts en silos protohistoriques au confluent SeineYonne (Seine-et-Marne) », Revue archéologique du centre de la France, 39, p. 5-30.

\section{DENAIRE A.}

2007: «Les sépultures multiples du Néolithique récent de Didenheim/ Morschwiller-le-Bas (Haut-Rhin)", in LE BRUN-Ricalens F., VAlotTEAU F., HAUZEUR A. (DIR.), Relations interrégionales au Néolithique entre Bassin parisien et Bassin rhénan, Actes du $26^{e}$ colloque interrégional sur le Néolithique, Luxembourg, 8-9 nov. 2003, Luxembourg, Musée national d'histoire et d'art (coll. Archeologia Mosellana, 7), p. 567-583.

2009: «Radiocarbon Dating of the Western European Neolithic: Comparison of the Dates on Bones and Dates on Charcoals», in «Actes du $5^{\mathrm{e}}$ symposium international sur le radiocarbone et l'archéologie, Zurich, 26-28 mars 2007», Radiocarbon, 51,2 , p. $657-674$.

\section{DENAIRE A, JEUNESSE C.}

2008: «Trois nouvelles tombes du Néolithique moyen (cultures de Grossgartach et de Roessen) découvertes à Réguisheim "Oberfeld/Grossfeld" et Sierentz "ZAC Hoell" (Haut-Rhin)", Cahiers alsaciens d'art, d'archéologie et d'histoire, 51, p. 3-13.

\section{DiECKMANN B.}

1985: «Die neolithischen Ufersiedlungen von Hornstaad-Hörnle am westlischen Bodensee. Die Grabungskampagne 1983/1984 », in BECKER B., BILLAMBOZ A., DiECKMANN B., KoKABI M., KROMER B., LIESE-KLEIBER H., RÖSCH M., SChlichtherle H., STRAHM C. (DIR.), Berichte zu Ufer- und Moorsiedlungen Südwestdeutschlands -2- Materialhefte zur Vor- und Frühgeschichte in BadenWürttemberg, 7, p. 98-124.

\section{DieckManN B., HaRWATH A.} HOFFSTADT U.

2007: Hornstaad-Hörnle IA: die Befunde einer jungneolithischen Pfahlbausiedlung am westlichen Bodensee, Siedlungsarchäologie 
im Alpenvorland IX/Forschungen und Berichte zur Vor- und Frühgeschichte in Baden-Württemberg, 98, Stuttgart, Konrad Theiss, 480 p.

DÖHLE H.-J., WAGNER K., WEIGELT J.

1992: «Eine Opfergrube der Baalberger Kultur von Alsleben, Landkreis Bernburg », Jahreschrift für Mitteldeutsche Vorgeschichte, 75, p. 51-69.

DUDAY H., VAQUER J.

2003: «Les sépultures chasséennes du site des Plots, Berriac (Aude)», in CHAMBON P. LECLERC J. (DIR.), Les Pratiques funéraires néolithiques avant 3500 av. J.-C. en France et dans les régions limitrophes, Paris, SPF (coll. Mémoires de la SPF, 33), p. 73-79.

\section{EICH-FRANCKE E.}

1967: Die Funde der Michelberger Kulture aus dem westlischen Oberrheingebiet, Zeitschrift der, Verlag Stadtbibliothek Worms, 22, $240 \mathrm{p}$.

\section{FISCHER U.}

1956: Die Gräber der Steinzeit im Saalegebiet: Studien über neolithische und frühbronzezeitliche Grab- und Bestattungsformen in SachsenThüringen, Berlin, Walter de Gruyter (Vorgeschichtliche Forschungen, 15), $327 \mathrm{p}$.

FORRER R.

1911: «Das Neolitische Gräberfeld bei Lingolsheim», Anzeiger für Elsässische Altertumskunde, 9-10, p. 149-171.

1912: «Ein neolithischer Pfahlbau bei Erstein-Murgiessen und die verwandten Fundstellen im Elsass", Anzeiger für Elsässische Altertumskunde, 13-14, p. 243267.

1922: «Nouvelles découvertes et acquisitions du musée préhistorique et gallo-romain de Strasbourg", Anzeiger für Elsässische Altertumskunde, 49-52, p. 1-34.

1938: "Le cimetière de Lingolsheim à poteries poinçonnées, au crâne trépané et aux tombes de la zone rubanée", Cahiers alsaciens d'archéologie, d'art et d'histoire, 111116, p. 191-206.

\section{GALlAY A.}

2006: Les Sociétés mégalithiques: pouvoir des hommes, mémoire des morts, Lausanne, Presses polytechniques et universitaires romandes (coll. Le Savoir suisse, 37), 139 p.

\section{GALLAY M.}

1970: Die besiedlung der südlichen Oberrheinebene in Neolithikum und Frühbronzezeit, Badische Fundberichte, 12, 199 p.
HENIGFELD Y.

2005: Eckbolsheim «Parc d'Activités» (Bas-Rhin), DFS de fouille de sauvetage, Strasbourg, SRA Alsace.

JEUNESSE C.

1982: La Culture de Michelsberg en Alsace: essai de synthèse, Mémoire de Maîtrise, Université de Strasbourg, 150 p.

1989: «La culture de Munzingen dans le cadre du "Jungneolithikum" du sudouest de l'Europe centrale d'après les découvertes récentes des sites alsaciens de Didenheim (Haut-Rhin) et Geispolsheim (Bas-Rhin)", Geispolsheim, Cahiers de l'APRAA, 5, p. 155-184.

1992: Il y a 7000 ans l'Alsace: le site archéologique du lotissement Sainte-Odile à Rosheim (BasRhin) et les premiers agriculteurs de la plaine d'Alsace, Catalogue d'exposition, Rosheim, 19-21 mars 1992 et Strasbourg, 2-25 avril 1992, Zimmersheim, APRAA, $75 \mathrm{p}$.

1998: «À propos de la signification historique des dépôts dans le Néolithique danubien ancien et moyen", in Tradition und Innovation, Festschrift für C. Strahm, Rahden, Verlag Marie Leidorf, p. 31-50.

2004: «Les traditions funéraires du Néolithique moyen en Europe centrale dans le cadre du système funéraire danubien ", in Alt K., ARbogast R.-M., JEUnESSE C., VAN WILLIGEN S. (DIR.), Archéologie funéraire du Néolithique danubien: nouveaux enjeux, nouvelles approches, Actes de la table ronde de Fribourg-en-Brisgau, 17-18 oct. 1998, Geispolsheim, APRAA (coll. Cahiers de l'APRAA, 20), p. 3-26.

2010: «Les sépultures en fosses circulaires de l'horizon 4500-3500: contribution à l'étude comparée des systèmes funéraires du Néolithique européen", in BARAY L., BOULESTIN B. (DIR.), Morts anormaux et sépultures bizarres: les dépôts humains en fosses circulaires ou en silos du Néolithique à l'âge du Fer, Actes de la table ronde interdisciplinaire de Sens, mars-avril 2006, Dijon, Éditions universitaires de Dijon, p. 26-48.

Jeunesse C., Lefranc P., Denaire A.

2003: Groupe de Bischheim, origine $d u$ Michelsberg, genèse du groupe d'Entzheim: la transition entre le Néolithique moyen et le Néolithique ancien dans les régions rhénanes, Geispolsheim, APRAA (coll. Cahiers de l'APRAA, 18-19), $280 \mathrm{p}$.

JeUnesse C., SAInty J.

1986: «Un nouvel habitat du Michelsberg récent (groupe de Munzingen) à Geispolsheim (Bas-Rhin), $1^{\text {re }}$ partie: les structures", Cahiers de l'APRAA, 2, p. 37-71.

1987: «Un nouvel habitat du Michelsberg récent (groupe de Munzingen) à Geispolsheim (Bas-Rhin), 2e partie: les mobiliers ", Cahiers de l'APRAA, 3, p. 88-129.

\section{KIMMIG W.}

1941-1947: «Neue Michelsbergfunde am Oberrhein", Badische Fundberichte, 17, p. $95-127$

1950: «Zur Frage der Rössener Kulturen südlichen Obenheim ", Badische Fundberichte, 18, p. 42-62.

\section{KLASSEN L.}

2001 : Frühes Kupfer in Norden. Untersuchungen zur Chronologie, Herkunft und Bedeutung der Kupferfunde der Nordgruppe der Trichterbecherkultur, JutlandAarhus University Press (coll. Jutland Archaelogical Society, 36), $385 \mathrm{p}$.

\section{KLASSEN L., PÉtrequin P., GRUT H.}

2007: «Haches plates en cuivre dans le Jura français: transferts à longue distance de biens socialement valorisés pendant les $\mathrm{IV}^{\mathrm{e}}$ et III ${ }^{\mathrm{e}}$ millénaires ", Bulletin de la SPF, 104, 1, p. 101-124.

\section{KOCH R.}

1971: «Zwei Erdwerke der Michelsberger Kultur aus dem Kreis Heilbronn ", Fundberichte aus Schwaben, 19, p. 51-67.

KUBENZ T.

1994: «Baalberger Kultur», in BEIER H.-J., EINICKE R. (DIR.), Das Neolithikum im Mittelelbe-Saale-Gebiet und in der Altmark, Beiträge zur Ur- und Frügeschichte Mitteleuropas, 4, p. 113-127.

KÜHNLE G., WIECHMANN A., Arbogast R.-M., BoËs E., Croutsch C. 1999-2000: "Le site Michelsberg et Munzingen de Holtzheim (Bas-Rhin)", Revue archéologique de l'Est, 50, p. 3-51.

\section{KREINER L.}

1995: «Grabfunde der Münchshöfener Kultur im Landkreis DingolfingLandau", in SCHMOTZ K. (DIR.), Vorträge Niederbayerischer Archäologentag, Deggendorf, 13, p. 71-84

\section{KREINER L., VAN DEN DRIESCH A.}

1992: «Ein Dorf mit Zeremonial Gruben des Facies Wallerfing aus Mamming, Landkreis Dingolfing-Landau, Niederbayern ", Das Archäologische Jahr in Bayern, p. $37-39$.

\section{LAMBACH F}

1986: «Les sépultures Michelsberg d'Alsace: quelques données nouvelles à propos des 
rites funéraires", Cahiers de l'APRAA, 2, p. 16-36.

\section{LANCHON Y., BRUNET P., BRUNET V., CHAMBON P.}

2006: «Fouille de sauvetage d'un monument funéraire et d'une enceinte néolithiques à Vignely "la Noue Fenard" (Seine-etMarne) », in DUHAMEL P. (DIR.), Impacts interculturels au Néolithique moyen, du terroir au territoire: sociétés et espaces, Actes du $25^{e}$ colloque interrégional sur le Néolithique, Dijon, 20-21 oct. 2001, Université de Dijon, Société archéologique de l'Est (Suppl. à la Revue archéologique de l'Est, 25), p. 335351

\section{LANGe G., Poulain T.}

1984: «Le camp chasséen du mont d'Huette à Jonquières (Oise) -V- Les vestiges humains", in Le Néolithique dans le nord de la France et le Bassin parisien, Actes du colloque interrégional sur le Néolithique, Compiègne, 14-26 sept. 1982, Revue archéologique de Picardie, 1-2, p. 265268.

\section{LASSERRe M., BoËS E., GEORGE P.}

1999: "L'enceinte néolithique à dépôts humains de Holtzheim, les Sablières Réunies (Bas-Rhin) ", Cahiers de l'APRAA, $15,153-175$.

LEFRANC $P$.

2001: "L'habitat néolithique moyen et récent de Holtzheim "Altmatt"/Zone d'activités économiques-phase 3 (BasRhin) (fouilles 2000 et 2001)", Cahiers de l'APRAA, 17, p. 107-134

LEFRANC P., ARBOGAST R.-M., BOËS E.

2007: «L'habitat néolithique récent de Rosheim "Leimen" (Bas-Rhin)», Cahiers alsaciens d'archéologie d'art et d'histoire, 50, p. 11-26.

\section{LEFRANC P., BOËS E.}

2006: Rosheim "Leimen» (Bas-Rhin): occupations du Néolithique récent, du début $d u$ Bronze moyen et du premier âge du Fer, DFS de fouille de sauvetage, Strasbourg, SRA Alsace, 141 p., 51 pl.

Lefranc P., Bö̈S E., Croutsch C.

2009: «Une nécropole de la transition $\mathrm{V}^{\mathrm{e}}$-IV $\mathrm{e}$ millénaires à Ungersheim (HautRhin) ", Bulletin de la SPF, 106, 2, p. 313327.

Lefranc P., Chenal F.

2010: Colmar-Aérodrome (Haut-Rhin): occupations du Néolithique récent (culture de
Munzingen) et de la Protohistoire, DFS, SRA Alsace/Inrap, 328 p.

Lefranc P., Denaire A., BoËs E.

À paraître: "Le site Néolithique ancien et moyen d'Ittenheim (Bas-Rhin)", Revue archéologique de l'Est, 59.

\section{Lefranc P., JeUnesse C.}

2001: «L'habitat néolithique moyen (groupe de Bruebach-Oberbergen et groupe d'Entzheim) et récent (Munzingen) d'Ensisheim THK", Cahiers de l'APRAA, 17, p. 69-89.

\section{LICHARDUS J.}

1986: «Le rituel funéraire de la culture de Michelsberg dans la région du Rhin supérieur et moyen », in DEMOULE J.-P., GUILAInE J. (DIR.), Le Néolithique de la France, hommage à G. Bailloud, Paris, Picard, p. 343-348.

\section{LICHARDUS-ITTEN M.}

1980 : Die Gräberfelder der Grossgartach Gruppe in Elsass, Bonn, R. Habelt (coll. Saarbrücker Beiträge zur Altertumskunde, 25), $220 \mathrm{p}$.

1991: «Hortfunde als Quellen zum Verständnis der frühen Kupferzeit", in LICHARDUS J. (DIR.), Die Kupferzeit als historische Epoche: eine forschungsgeschichtliche Einleitung, Symposium Saarbrücken und Otzenhausen, 6-13 nov. 1988, Bonn, Rudolf Habelt verlag, Saarbrücker Beiträge zur Altertumskunde, 55, p. 753-765.

\section{LOISON G., FABRE V., VILLEMEUR I.}

2003: «Structures domestiques et aménagements funéraires sur le site chasséen du Crès à Béziers (Hérault)», Archéopages, 10, p. 32-39.

\section{LÜNING J.}

1968: «Die Michelsberger Kultur. Ihre Funde in zeitliche und räumliche Gliedernug", Bericht der Römisch-Germanischen Kommission, 48, 350 p.

\section{MAIER R.-A.}

1965: "Michelsberg-Altheimer Skelettgruben von Inningen bei Augsburg in BayerischSchwaben», Germania, 43, 1, p. 8-17.

\section{MARIÉTHOZ F.}

2007: "Variabilité des pratiques funéraires en Valais autour de 4000 av. J.-C», in Moinat P., Chambon P. (DIR.), Les Cistes de Chamblandes et la place des coffres dans les pratiques funéraires du Néolithique moyen occidental, Actes du colloque de Lausanne, 12-13 mai 2006, Lausanne, Cahiers d'archéologie romande (coll. Cahiers d'ar- chéologie romande, 110 et Mémoires de la SPF, 43), p. 265-276.

\section{MAROLLE C.}

1989: «Le village Michelsberg des "Hautes Chanvières" à Mairy (Ardenne) -I- Étude préliminaire des principales structures", Gallia Préhistoire, 31, p. 93-118.

\section{MAUSS M.}

1924-1925 (rééd. 2007) : Essai sur le don, Paris, PUF.

\section{MÉNIEL P.}

1987: «Les dépôts d'animaux du fossé chasséen de Boury-en-Vexin (Oise) », Revue archéologique de Picardie, 1-2, p. 3-26.

Moorrees C. F. A., FANNING E. A., HUNT E. E. J.

1963a: «Formation and Resorption of three Deciduous Teeth Children", American Journal of Physical Anthropology, 21, p. 205213.

1963b: «Age Variation and Formation Stages for ten Permanent Teeth", Journal of Dental Research, 42, p. 1490-1502.

Murail P., Bruzek J., houët F., Cunha E. 2005: «DSP: un outil de diagnose sexuelle probabiliste à partir des données métriques de l'os coxal», Bulletins et mémoires de la Société d'anthropologie de Paris, 17, p. 167-176.

\section{NIGKEL C.}

1998: «Menschliche Skelettreste aus Michelsberger Fundzusammenhängen: zur Interpretation einer Fundgattung», Bericht der Römisch-Germanischen Kommission, 78, p. 29-195.

\section{NOBIS G.}

1977: «Die Fauna», in LÜNING J., ZÜRN H. (DIR.), Die Schussenrieder Siedlung im «Schlosslesfeld» Markung Ludwigsburg, Stuttgart, Muller und Graff (coll. Forschungen und Berichte zur Vor- und Frühgeschichte in Baden-Württemberg, 8), p. 82-90.

\section{OTTAWAY B., STRAHM C.}

1975: «Swiss Neolithic Copper Beads: Currency, Ornament or Prestige Items?", World Archeology, 6, p. 307-321.

OWIngs-Webb P. A., SUChey J. M.

1985: «Epiphyseal Union of the Anterior Iliac Crest and Medial Clavicule in a Modern Multiracial Sample of American males and females", American Journal of Physical Anthropology, 68, p. 457-466. 


\section{PERRIN B.}

2006: Les Ossements humains situés dans les fossés d'enceintes de la culture Michelsberg, Mémoire de Master II, Université de Strasbourg, 2 vol.

\section{PÉtReQuin P.}

1972: La Grotte de la Tuilerie à Gondenans-lesMontby, Paris, Les Belles Lettres (coll. Annales littéraires de l'université de Besançon, 137), 171 p.

\section{Pétrequin P., Cassen S., Croutsch C. ERRERA M.}

2002: «La valorisation sociale des longues haches dans l'Europe néolithique», in GUILAINe J. (DIR.), Matériaux, productions, circulations du Néolithique à l'âge du Bronze, Paris, éd. Errance, p. 67-98.

\section{PÉTREQUiN P., JEUNESSE C.}

1995: La Hache de pierre: carrières vosgiennes et échanges de lames polies pendant le Néolithique (5400-2100 av. J.-C.), Paris, éd. Errance, $131 \mathrm{p}$.

\section{Pétrequin P., Pétrequin A.-M.}

1993: Écologie d'un outil: la hache de pierre en Irian Jaya (Indonésie), Paris, éd. du CNRS (coll. Monographie du CRA, 12), $444 \mathrm{p}$.

\section{Poulain T.}

1977: «Fosses et sépultures Michelsberg, sablière Maetz à Rosheim (Bas-Rhin): étude de la faune ", Bulletin de la SPF, 74, 2, p. 608-621.

1987: «L'habitat néolithique récent (groupe de Munzingen) de Geispolsheim (BasRhin), lieux-dits Bruechel et Kirstenfeld: étude de la faune ", Cahiers de l'APRAA, 3, p. 136-144.

\section{PREUSS J.}

1966: Die Baalberger Gruppe, in Mittel deutschland, Berlin, Veröffentlichungen des Landesmuseum für Vorgeschichte in Halle, 21, 253 p., 33 pl.

\section{ROHMER M.}

1995: Rosheim "Rosenmeer», DFS de fouille de sauvetage, Strasbourg, SRA Alsace, 10 p., 28 pl.

SANGMEISTER E., STRAHM C.

1974: «Die Funde aus Kupfer in Seeberg Burgäschisee-Sud », Berne, Acta Bernensia II, 6 , p. 189-259

\section{SAUER C.}

1959: «Trouvailles néolithiques sur l'aérodrome d'Entzheim (Bas-Rhin)", Cahiers alsaciens d'archéologie d'art et d'histoire, 3, p. 5-8.

\section{SCHAEFFER F.-A.}

1926: «Sépultures d'accroupis et caves de cabanes néolithiques d'Achenheim", Cahiers alsaciens d'histoire et d'art, 61-68, p. $273-285$

\section{SCHEUER L., BLACK S.}

2000: «Development and Aageing of the Juvenile Skeleton", in COX M., MAYS S. (DIR.), Human Osteology in Archaeology and Forensic Science, London, Greenwich Medical Media, p. 9-22.

\section{SCHMID E.}

1958: «Die Nebenfunde auf dem Munzinger Berg", Badische Fundberichte, 21, p. 51-55.

\section{SCHMitT G.}

1974: «La transition entre le Néolithique moyen et le Néolithique final en BasseAlsace", Revue archéologique de l'Est, 25, p. $277-364$.

\section{SCHNEIKERT F.}

2006: Entzheim "Zone d'activités» (Alsace, Bas-Rhin), DFS de fouille de sauvetage, Strasbourg, SRA Alsace, 60 p.

\section{SCHRÖTER P.}

1996: «Eine Münchshöfener Gruppenbestattung aus Grossmehring, Lankreis Eichstätt, Oberbayern", Archäologische Jahr im Bayern, p. 36-40.

\section{SGHWEITZER J.}

1987: «Le site Michelsberg de Didenheim», Cahiers de l'APRAA, 3, p. 50-87.

\section{SCHWEITZER J., FULLERINGER R.}

1973: "Découverte de fosses Michelsberg à Riedisheim", Bulletin du Musée historique de Mulhouse, 81, p. 23-38.

\section{SEIDEL U.}

2005: "Das Michelsberger Erdwerk von Heilbronn-Klingenberg Schlossberg", Fundberichte aus Baden-Württemberg, 28 , 1, p. 19-61.

\section{SELLIER P.}

1996: «La mise en évidence d'anomalies démographiques et leur interprétation: population, recrutement et pratiques funéraires du tumulus de Courtesoult», in PININGRE J.-F. (DIR.), Nécropoles et sociétés au premier âge du Fer: le tumulus de Courtesoult, Paris, éd. de la Maison des sciences de l'homme (coll. Documents d'archéologie française, 54), p. 188-202.

\section{STAUCH E., BANGHARD K.}

2002: «Das ganz normale Michelsberg, Neues zur Jungneolithischen Siedlungsgeschichte zwischen Rhein und Neckar», in ETTEL P. (DIR.), Interdisziplinäre Beiträge zur Siedlungsarchäologie, Gedenkschrift für Walter Janssen, Rahder, Verlag Marie Leidorf, p. 369-390.

\section{STEPPAN K.}

1998: «Archäozoologische Untersuchung der Säugetierreste aus den Gräben der Michelsberger Erdwerke in Bruchsal, Landkreis Karlsruhe: die Bedeutung der Haus und Wildsäugetiere im Rahmen der jungneolithischen Ernährungswirtschaft in Südwestdeutschland", in Die Michelsberger Kultur und ihre Randgebiete: Probleme der Entstehung, Chronologie und des Siedlungswesens, Kolloquium Hemmenhofen, 21-23 févr. 1997, Stuttgart, Theiss, Materialhefte zur Archäologie in BadenWürttemberg, 43, p. 143-150.

2003: «Taphonomie-Zoologie-ChronologieTechnologie-Ökonomie: die Säugetierreste aus den jungsteinzeitlichen Grabenwerken in Bruchsal/Landkreis Karlsruhe», Stuttgart, Theiss, Materialhefte zur Archäologie in Baden-Württemberg, $66)$, p. 3-244

STIEbER A.

1956: «Stations néolithiques d'Alsace», Bulletin de la SPF, 53, p. 750-758.

\section{STOCKL H., NEUbAUER-SAURER D.}

1990: «Neue Funde der Strassburger und Wauwiler Gruppe aus dem nördlichen Kaiserstuhlvorland", in Wawil, Bruebach, Entzheim, Strassburg... Les groupes à «kugelbecher» dans le sud de la plaine du Rhin supérieur (4500-4100 av. J.-C.) (coll. Cahiers de l'APRAA, 6), p. 115-170.

\section{TESTART A.}

2001: L'Esclave, la dette et le pouvoir: étude de sociologie comparative, Paris, éd. Errance, $238 \mathrm{p}$.

2004: Les Morts d'accompagnement: la servitude volontaire, Paris, éd. Errance, 2 vol., 262 p. et $137 \mathrm{p}$.

THÉVENET C.

2007: «De pierre ou de bois: coffre et architecture de la sépulture $10 \mathrm{du}$ monument Michelsberg de Beaurieux (Aisne, France)», in CHAMBON P., MOINAT P. (DIR.), Les Cistes de Chamblandes et la place des coffres dans les pratiques funéraires du Néolithique moyen occidental, Actes du colloque de Lausanne, 12-13 mai 2006, 
Lausanne/Paris, SPF (coll. Cahiers d'archéologie romande, 110 et Mémoire de la SPF, 43), p. 143-153.

Thévenin A., Sainty J., Poulain T.

1978: «Fosses et sépultures Michelsberg, sablière Maetz à Rosheim (Bas-Rhin)", Bulletin de la SPF, 74 (coll. études et travaux, 2), p. 608-621.

\section{TOULLEC C.}

2008: Matzenheim «Le Lavoir», DFS de fouille de sauvetage, Strasbourg, SRA Alsace, 2 vol., 94 p., 75 pl. et annexes.

\section{TOUSSAINT M., BECKER A.}

1994: «Une sépulture du Michelsberg: le trou de la Heid à Comblain-au-Pont (province de Liège, Belgique) », Bulletin de la $S P F, 91,1$, p. 77-84.

\section{Toussaint M., Collet H., VAN DeR} LINDEN M.

1997: «Découverte d'un squelette humain dans le puits de mine néolithique ST11 de Petit-Spiennes (Hainaut)», Notae Praehistoricae, 17, p. 213-219.

TrefFort J.-M., DUMONT A.

2000: Merxheim «Trumelmatten»(Haut-Rhin), Néolithique, Bronze final, Hallstatt et haut Moyen Âge, DFS de fouille de sauvetage, Strasbourg, SRA Alsace, 65 p., 56 fig., 16 photos et annexes.

\section{Tresset A.}

1988: "La faune néolithique de Noyen-surSeine (Seine-et-Marne) », Anthropozoologica, 8, p. 12-14.

1996: Le Rôle des relations homme/animal dans l'évolution économique et culturelle des sociétés des $V^{E}$ et $I V^{E}$ millénaires en Bassin parisien, Thèse de Doctorat, Université Paris-IPanthéon-Sorbonne, 3 vol.

\section{Tristan C.}

2004: Marlenheim (Bas-Rhin), contournement routier: deux habitats rubanés et une occu- pation hallstattienne, DFS de fouille de sauvetage, Strasbourg, SRA Alsace, $77 \mathrm{p}$.

\section{VILLES A.}

1986: «Une hypothèse: les sépultures de relégation dans les fosses d'habitat protohistoriques en France septentrionale », in DUDAY H., MASSET C. (DIR.), Anthropologie physique et archéologie: méthodes d'étude des sépultures, Actes du colloque de Toulouse, nov. 1982, Paris, éd. du CNRS, p. 167-174.

\section{WOLF J-J.}

1979: "Nouveaux éléments du Michelsberg à Eschentzwiller et Magstatt-le-Bas", Bulletin du Musée historique de Mulhouse, 76, p. 29-38.

\section{Zehner M., Denaire A., BAKaj B.}

2002: Mulhouse Rocade-Ouest (Haut-Rhin), DFS de fouille de sauvetage, Strasbourg, SRA Alsace, $169 \mathrm{p}$. 